High-resolution reservoir characterization by seismic inversion with geological constraints

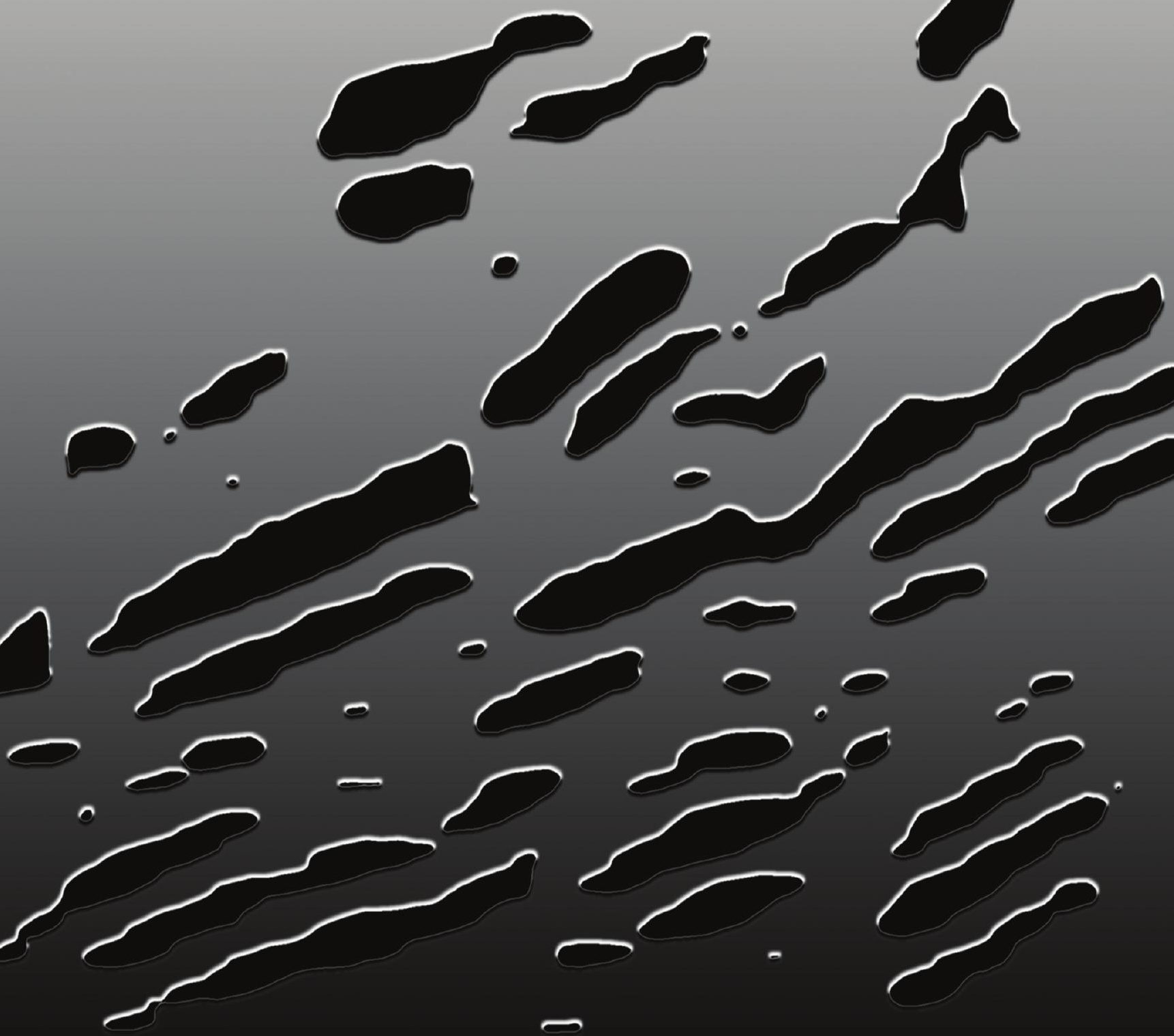

Daria Tetyukhina 
High-resolution reservoir characterization by seismic inversion with geological constraints 



\section{High-resolution reservoir characterization by seismic inversion with geological constraints Proefschrift}

ter verkrijging van de graad van doctor aan de Technische Universiteit Delft, op gezag van de Rector Magnificus prof. ir. K. C. A. M. Luyben, voorzitter van het College voor Promoties in het openbaar te verdedigen op dinsdag 14 september 2010 om 12:30 uur door Daria TETYUKHINA

Master of Science in Geology (Lomonosov Moscow State University) geboren te Moskou (Rusland). 
Dit proefschrift is goedgekeurd door de promotoren:

Prof. dr. S.M. Luthi,

Prof. dr. ir. L.J. van Vliet,

Prof. dr. ir. C.P.A. Wapenaar.

Samenstelling promotiecommissie:

Rector Magnificus, voorzitter

Prof. dr. S.M. Luthi, Technische Universiteit Delft, promotor

Prof. dr. ir. L.J. van Vliet, Technische Universiteit Delft, promotor

Prof. dr. ir. C.P.A. Wapenaar, Technische Universiteit Delft, promotor

Prof. dr. ir. A. Gisolf, Technische Universiteit Delft

Prof. dr. S.A. Petersen, Statoil ASA

Dr. ir. P.M. Mesdag, Fugro-Jason Netherlands BV

Dr. A.M. Schwab, Marathon International Petroleum

\section{SUPPORT}

This work is financially supported by Research Center Delft Earth and Statoil.

ISBN 978-94-6113-019-8

Copyright (c) 2010 by Daria Tetyukhina, Delft University of Technology, Delft, The Netherlands.

All rights reserved. No part of this book may be reproduced, in any form or by any means, without prior permission in writing from the author.

Printed by Ipskamp Drukkers, The Netherlands. 
To my family 



\section{Contents}

Contents vii

Summary xi

Samenvatting $\quad$ xv

1 Introduction 1

1.1 Introduction to Inversion . . . . . . . . . . . . . 2

1.2 Choice of Geological Objects . . . . . . . . . . 5

1.3 Research Challenges . . . . . . . . . . . . 6

1.3.1 Statement of the Problem . . . . . . . . 6

1.3.2 Research Objectives . . . . . . . . . . . 8

1.4 Outline of the Thesis . . . . . . . . . . . . 9

2 Theoretical Background 11

2.1 The forward and inverse problem . . . . . . . . 11

2.1.1 Forward modeling - 1D convolution model . . 13

2.1.2 Existence and non-uniqueness of solution . . . . 14

2.2 Theory of seismic inversion . . . . . . . . . . . 17

2.2 .1 About Bayes . . . . . . . . . . . . 17

2.2.2 Bayes' rule as a basis for inverse problems . . . 19

2.2.3 The likelihood function . . . . . . . . . . . . 19 
2.2.4 A priori information . . . . . . . . . 20

2.2 .5 Uncertainties . . . . . . . . . . . . . . 21

2.2 .6 Point Estimation . . . . . . . . . . . . . . 21

2.2.7 Nonlinear least-squares estimation . . . . . . . . 22

2.2.8 The probability density function selection . . . 23

2.3 Regularization . . . . . . . . . . . . . . . . 25

2.4 Optimization . . . . . . . . . . . . . . . . 28

2.4.1 Simplex method . . . . . . . . . . . . 30

2.4.2 Gradient-based method . . . . . . . . . . 31

3 Field Data Set 33

3.1 Clinoform Systems . . . . . . . . . . . . . . 33

3.1 .1 Basin architecture . . . . . . . . . . . 35

3.1.2 The log response in clinoform systems . . . . . 37

3.2 Geological Framework . . . . . . . . . . . . . . . 41

3.3 Seismic Data . . . . . . . . . . . . . . . . . 45

3.4 Well Data . . . . . . . . . . . . . . . 46

3.5 Data Interpretation . . . . . . . . . . . . . . . 48

3.5.1 Clinoform Mapping . . . . . . . . . . . . . 53

3.6 Seismic Data Selection for Inversion . . . . . . . . . . 54

3.6.1 Reference Reflections . . . . . . . . . . . 54

3.6.2 Proximity of Wells . . . . . . . . . . . 55

3.7 Wavelet Extraction . . . . . . . . . . . . . 60

4 Stratigraphic model-based, low-parametrization seismic inversion 63

4.1 Introduction . . . . . . . . . . . . . . 63

4.2 1D Inversion . . . . . . . . . . . . . . . . . . . 64

4.2.1 Formulation of the inverse problem . . . . . . 64

4.2 .2 A priori Information . . . . . . . . . . 65

4.2 .3 Initial Model . . . . . . . . . . . . . . 66

4.2 .4 Optimization ................ . . 71 
4.2.5 Regularization. . . . . . . . . . . . 71

4.2 .6 Results. . . . . . . . . . . . . . . 73

4.3 Clinoform Geometry Estimation . . . . . . . . . 78

4.3.1 Geological Model . . . . . . . . . . . . . . 78

4.3.2 Mathematical Model . . . . . . . . . . . . . . 79

4.3.3 Formulation of the inverse problem . . . . . . 79

4.3.4 Optimization . . . . . . . . . . 80

4.3.5 Initial Model . . . . . . . . . . . . . . . . 81

4.3 .6 Results . . . . . . . . . . . . . . 81

4.4 Inversion of the Clinoforms . . . . . . . . . . . 84

4.4.1 Formulation of the inverse problem . . . . . . 84

4.4.2 Structural Information Incorporation . . . . . . 87

4.4.3 Regularization . . . . . . . . . . . . . . 88

4.4.4 Results . . . . . . . . . . . . . . . . . 88

5 Grid-based Seismic Inversion $\quad 95$

5.1 Introduction . . . . . . . . . . . . . . . 95

5.2 Mathematical Background . . . . . . . . . . 96

5.2.1 Super Resolution Reconstruction of Small Moving Objects . . . . . . . . . . . . . 96

5.2 .2 Total Variation Criterion . . . . . . . . . . . 97

5.2 .3 Optimization . . . . . . . . . . . . . 98

5.3 1D Inversion of the Clinoforms . . . . . . . . . . . 99

5.3.1 Formulation of the Inverse Problem . . . . . . . 99

5.3 .2 Vertical Operator . . . . . . . . . . . . 101

5.3 .3 Regularization . . . . . . . . . . . . . 104

5.3.4 Results. . . . . . . . . . . . . . . . . 119

5.4 Inversion of the Clinoforms . . . . . . . . . . 120

5.4.1 Formulation of the Inverse Problem . . . . . . 120

5.4 .2 Horizontal Operator . . . . . . . . . . . . . 122

5.4 .3 Regularization . . . . . . . . . . . . . . 123

5.4 .4 Results. . . . . . . . . . . . . . 134 
6 Discussion, conclusions, and recommendations

6.1 Stratigraphic model-based, low-parametrization seismic inversion . . . . . . . . . . . . . . . . 141

6.2 Grid-based Seismic Inversion . . . . . . . . . . . . . 144

6.3 Geological Significance . . . . . . . . . . . . . . . 148

6.4 Recommendations for further research . . . . . . . . . 149

Bibliography

About the author

161

List of Publications

163

Acknowledgments 


\section{Summary}

\section{High-resolution reservoir characterization by seismic inversion with geological constraints}

Fluvio-deltaic sedimentary systems are of great interest for explorationists because they can form prolific hydrocarbon plays. However, they are also among the most complex and heterogeneous ones encountered in the subsurface. Reservoirs in clinoform systems are difficult to characterize because they show two main types of complexity: complex sedimentology and poor seismic imaging. The former is due to complex internal architecture with many small sedimentary elements often at a sub-seismic scale. Poor seismic imaging occurs because the internal layers of the clinoform often do not differ much in their acoustic properties, in addition, they have thicknesses that are below the vertical resolution of seismic data, and therefore such features do not show up very well on seismic images. Obviously, the most unfavorable situation occurs when both conditions interact.

The static model of a fluvio-deltaic (clinoform) reservoir is extremely important because it plays a critical role in the field development plan- 
ning. There are many ways to build a static model but the most effective way is by integrating seismic and well data through the construction of an acoustic impedance model by inversion of seismic data within a sequence stratigraphic framework.

There are several reasons to integrate well-log data into the inverse process in the reservoir characterization workflow, such as the integration of different sources of information in a common earth model, the estimation of the seismic distortion (also known as the wavelet filter), etc. In addition, the low vertical resolution of seismic data is an important motivation to integrate well-log information into the inverse process and thereby complement the relatively dense horizontal coverage of seismic data with high-resolution borehole data (Van Riel and Mesdag, 1988; Van Riel and Pendrel, 2000; Van Riel, 2000; Bosch et al., 2009).

Another potential benefit of seismic inversion is the ability to incorporate structural and stratigraphic information of the reservoir in order to differentiate between similar mathematical solutions on the basis of their geological viability.

We present two different inversion approaches for poststack, time migrated seismic data and apply them to a clinoform sequence in the North Sea.

Both inversion methods are not fully 2D, but more than a series of independently processed 1D inversions. To stress the enforced continuity along the geological structure, we use the name pseudo $2 \mathrm{D}$ inversion. The methods use well data as a priori constraints but differ in the way they incorporate structural information. One method uses a discrete layer model from the well that is then propagated laterally along the clinoform layers, which are modeled as sigmoids. The second 
method uses a constant sampling rate from the well data and employs horizontal and vertical regularization parameters for lateral propagation. Both methods obtain an acoustic impedance image with a high level of detail. The first method has a low level of parameterization embedded in a geological framework and is computationally fast. The second method has a much higher degree of parameterization but is flexible enough to detect deviations in the geological settings of the reservoir, however there is no explicit geological significance and it is computationally much less efficient. Forward seismic modeling of the two inversion results indicates a good match of both methods with the actual seismic data.

The methods are especially considered to be useful when seismic data alone do not reveal the actual detailed reservoir architecture, which can be the case either because of their low vertical resolution or exceedingly thin layering. 


\section{Samenvatting}

\section{Hoge-resolutie reservoir karakterisatie}

\section{door seismische inversie met geologische}

voorwaarden

Fluvio-delta sedimentaire systemen zijn van groot belang voor exploratie-geofysici omdat ze producerende koolwaterstoffen vormen. Echter, ze behoren ook tot de meest complexe en meest heterogene systemen die men aantreft in de ondergrond. Reservoirs in een clinoform sequentie zijn moeilijk te karakteriseren vanwege twee complicerende factoren: complexe sedimentologie en slechte seismische beeldvorming. De eerste factor is te wijten aan de complexe interne architectuur met veel kleine sedimentaire elementen, vaak op een sub-seismische schaal. Slechte seismische beeldvorming is een gevolg van het feit dat de akoestische eigenschappen van de interne lagen van een clinoform onderling vaak niet veel verschillen. Bovendien liggen de laagdiktes veelal onder de vertikale resolutie van de seismiek en daarmee verschijnen dergelijke interne structuren niet goed op de seismische beelden. Vanzelfsprekend is de meest ongunstige situatie wanneer beide eigenschappen tegelijk optreden. 
Het statische model van een fluvio-delta (clinoform) reservoir is zeer belangrijk, omdat het een essentiële rol speelt in de planning om een veld te ontwikkelen. Er zijn veel manieren om een statisch model te bouwen, maar de meest effectieve combineert seismiek en put-data bij het bouwen van een akoestisch impedantie model door middel van inversie van de seismische data binnen een sequentieel stratigrafisch kader.

Er zijn verschillende redenen om log-gegevens van putten te gebruiken tijdens het inversie proces in de reservoir karakterisatie procedure, bijvoorbeeld de integratie van verschillende put-gegevens in een gemeenschappelijk model van de aarde, de schatting van de seismische vervorming (ook wel bekend als het wavelet-filter), enz. Ook een belangrijke reden om log-gegevens uit de put te integreren in het inversie proces is de lage verticale resolutie van de seismiek, die samen met de hoge-resolutie boorput gegevens een aanvulling geeft op de relatief hoge horizontale dekking van de seismische data (Van Riel and Mesdag, 1988; Van Riel and Pendrel, 2000; Van Riel, 2000; Bosch et al., 2009).

Een ander potentieel voordeel van seismische inversie is de mogelijkheid om structurele en stratigrafische informatie over het reservoir mee te nemen, zodat onderscheid gemaakt kan worden tussen gelijkwaardige wiskundige oplossingen op basis van hun geologische relevantie.

We presenteren twee verschillende benaderingen voor inversie van poststack, tijdsgemigreerde seismische data en passen deze toe op een clinoform sequentie in de Noordzee.

Beide inversie methodes zijn niet volledig 2D, maar wel meer dan een reeks van onafhankelijk uitgevoerde 1D inversies. Om de opgelegde continuïteit langs de geologische structuur te benadrukken, gebruiken 
we de naam pseudo-2D inversie. De methodes gebruiken put-gegevens als a priori restrictie, maar verschillen van elkaar in de manier waarop zij de structurele informatie verwerken. De ene methode maakt gebruik van een discreet gelaagd model gebaseerd op de put-gegevens, die vervolgens lateraal worden gedistribueerd langs de lagen van de clinoform, die als sigmoïdes zijn gemodelleerd. De tweede methode maakt gebruik van een equidistante bemonstering van de put-gegevens en past horizontale en vertikale regularisatie toe voor de laterale distributie. Beide methodes leiden tot een akoestisch impedantie beeld met een hoge mate van detail. De eerste methode heeft een lage parametrisatiegraad ingebed in een geologisch kader en is snel te berekenen. De tweede methode heeft een hogere mate van parametrisatie, maar is flexibel genoeg om afwijkingen te detecteren in de geologische opbouw van het reservoir. Echter, deze methode maakt geen gebruik van explicite geologische kennis en is rekenkundig minder efficient. Voorwaarts seismisch modelleren uitgaande van de twee inversie resultaten geeft voor beide methodes een goede gelijkenis met de werkelijke seismiek.

De methodes zijn vooral van belang voor situaties waarin de seismiek alleen niet de daadwerkelijke gedetailleerde reservoir architectuur laat zien, hetzij vanwege de lage verticale resolutie, hetzij vanwege de extreem dunne gelaagdheid.

Daria Tetyukhina 


\section{Chapter 1}

\section{Introduction}

Three criteria should be fulfilled for oil and gas accumulations to occur, namely the presence of a porous reservoir rock with appropriate geological structure, an effective seal and a mature source rock. The search for oil and gas usually focuses on sedimentary basins since source rocks are always sedimentary rocks (Luthi, 2008).

Although estimates of the global hydrocarbon reserves have increased over time with improvement of technology, the rate of discovery per year still remains smaller than the rate of consumption. Most oil and gas fields that could be discovered by applying basic technologies, like seismic exploration, combined with conceptual understanding of hydrocarbon systems, seem to have been discovered by now. The application of these technologies to oil and gas resources which are trapped in the remaining reservoirs is not straightforward (Cacas et al., 2008).

The availability of a large amount of 2D and 3D seismic data and wells as well as sufficient computer potency nowadays, makes feasible a construction of a coherent and consistent geological model of the hydrocarbon reservoir using an advanced technology based on their 
interpretation and their integration.

Inversion of seismic data is a well known oil- and gas-industry tool used for refining structural interpretation and reservoir geometry, property prediction, as well as reservoir characterization.

\subsection{Introduction to Inversion}

The inference of subsurface properties from measured data is identified with the solution of a so-called 'inverse problem' and this topic has been under development by geophysicists since the origin of their profession. A growing body of theory, along with the ever increasing computer power, were the driving forces for the improvement of geophysical inversion methods. Until the early 1960s geophysical inversion was carried out almost exclusively within the geophysicist's brain. Since then, geophysical inversion methods became much more quantitative, versatile and efficient ${ }^{1}$.

Inversion is capable of handling different kinds of geophysical data such as seismic, potential field, borehole data etc. For every specific case of inversion the assumption is made that a specific physical law holds. For the case of seismic inversion, this law is the wave equation or one of its numerous approximations. Thus, algorithms based on this physical law enable us to invert the measured data for the subsurface characteristics, which gave rise to these observations in the first place.

According to Sheriff (2002), inversion can be defined as a technique solving for a spatial distribution of parameters which could have produced an observed set of measurements. For the seismic case, the

\footnotetext{
${ }^{1}$ This section contains excerpts from an overview paper on geophysical inversion by Treitel and Lines (2001)
} 
observations consist of the so-called physical signature of a subsurface structure: the structure's reflected (or scattered) as a wave field due to a seismic source signal.

The inversion problem is almost always linked to forward modeling. The 'forward problem', consists of the determination of the data that would be measured for a given subsurface configuration, under the assumption that given laws of physics hold. In the case of seismic exploration, forward modeling makes use of a mathematical relationship, such as the wave equation, to synthesize an earth model's response to an excitation of a pulse of acoustic energy. It is of course crucial to choose a proper model parameterization and a forward modeling procedure that can adequately describe the observations. Such models are defined by a set of parameters, which for the acoustic case, are layer velocities and layer densities. Forward modeling is then implemented with a numerical algorithm that produces a synthetic seismogram, such as a seismic ray tracer, a finite difference, or a finite-element wave-equation solver. Defining the number of model parameters to be used and their impact on the entire result is another critical issue. The choice of the 'right' model is crucial and depends on the exploration problem at hand. However, it has been shown in the literature that even assuming that the model choice is adequate, numerous problems still remain. In fact, Jackson (1972) referred to inversion as the 'interpretation of inaccurate, insufficient, and inconsistent data'.

Although seismic inversion in various forms has been around as a viable exploration tool for more than 40 years, the discussion on inversion nomenclature is still ongoing. A very good overview is given by Pendrel (2001), who tried to classify the seismic inversion approaches based on the form of the objective function to be minimized. Later, Merletti and Torres-Verdin (2006) classified all seismic inversion procedures that have been reported in the open literature into two main 
categories: geostatistical and deterministic methods. Geoscientists tend to use deterministic inversion methods as their first choice to characterize seismic data and to retrieve rock properties.

Due to the ill-posedness of inverse problems, the obtained solutions are not unique; even worse, there is an infinite number of solutions that satisfy the data within prescribed error bounds (Cary and Chapman, 1988).

In order to find the best geophysical and geological solution from the large number of available mathematical solutions, other conditions should be introduced (Van Riel and Pendrel, 2000).

Deterministic seismic inversion that employs Bayes' rule provides a simple framework that integrates the information from all available measurements into a consistent image of the reservoir, and constrains these solutions to be biased toward an a priori knowledge about the subsurface parameters. One of the ways to express those constraints is in the form of multidimensional probability density functions, whose dimensionality is equal to the number of parameters describing a given model (Duijndam, 1988). Prior probability density describing prior knowledge (or prejudices) about the model parameters is usually combined with a 'likelihood function', which is the misfit between the model response and the observed seismic data. A one-dimensional example of a prior probability density function is a Gaussian curve whose peak corresponds to the most likely value of a given model parameter. Its width determines the range of values that this model parameter can possess. According to Bayes' rule the peak (or peaks) of the resulting multidimensional a posteriori probability distribution to a given inverse problem should disclose the most likely set of model parameter values. These values produce a synthetic model response satisfying the observed seismic data within prescribed error bounds. 


\subsection{Choice of Geological Objects}

In this study, a simple yet common geological building block is used to develop a novel approach to seismic inversion: the clinoform. 'Clinoform' is a term originally introduced by Rich (1951) to describe the shape of a depositional surface at the scale of the entire continental margin.

In the current geologic literature, the term clinoform refers to stratal packages with oblique internal layering (Cattaneo et al., 2004). Clinoform systems are typical progradational patterns that occur over a wide range of scales and in a broad spectrum of depositional environments, from centimeters (like sand dunes) to many kilometers (such as entire continental shelves), all of which may be conducive to form potential reservoirs. In the study of modern continental margins, clinoforms are widely recognized as one of the fundamental building blocks of the stratigraphic record.

Large-scale clinoform geometries originate when supply exceeds the available accommodation and sediment tends to build basinwards. Clinoforms develop typically at the front of river deltas or in subaqueous deltas. Subaqueous deltas are characterized by an overall sigmoidal geometry in sections perpendicular to the shore, submerged offlap break (and lack of subaerial exposure of the topset), muddy lithology, and high sediment accumulation rates (Cattaneo et al., 2003). Changes in clinoform thickness, internal geometry, and style of superposition of multiple clinoforms provide information regarding long-term margin subsidence, sea-level change, and short-term fluctuations of sediment supply (Cattaneo et al., 2004). 


\subsection{Research Challenges}

\subsubsection{Statement of the Problem}

Reservoirs in clinoform systems are difficult to characterize because they show two main types of complexity: complex sedimentology and poor seismic imaging. The former is due to complex internal architecture with many small sedimentary elements often at a sub-seismic scale. Poor seismic imaging occurs because the internal layers of the clinoform often do not differ much in their acoustic properties, in addition, they have thicknesses that are below the vertical resolution of seismic data, and therefore such features do not show up very well on seismic images. Obviously, the most unfavorable situation occurs when both conditions interact.

The static model of a fluvio-deltaic (clinoform) reservoir is extremely important because it plays a critical role in the field development planning (Cacas et al., 2008). There are many ways to build a static model, from basic interpolation of well log data within a structural framework to more advanced procedures that incorporate seismic data. The basic interpolation method is prone to large errors, especially when the number of wells available is small. Wells may have been drilled into particularly good or poor reservoir rocks. Extrapolation of properties from such wells without any guidance concerning the lateral variation in quality of the reservoir rocks may lead to significant over- or underprediction of hydrocarbon volumes (Marzuki et al., 2000). The most effective way of building an integrated model from seismic and well data is probably through the construction of an acoustic impedance model by inversion of seismic data within a sequence stratigraphic framework (Cacas et al., 2008). Acoustic impedance is often related to reservoir properties such as porosity, lithology and pore fluids. However, an impedance model derived from deterministic inversion of seis- 
mic data can sometimes fail to meet all objectives of reservoir characterization; specifically the layers of interest may not be adequately resolved by the seismic impedance data. A dominant wavelength at reservoir depth in the order of several tens of meters is quite common for an exploration seismic survey (Yilmaz, 2000). This seriously limits the minimum layer thickness that still can be discerned in the seismic image. According to Widess (1973) and Badley (1985), the theoretical vertical resolution of seismic data is approximately a quarter of the wavelength.

There are several reasons to integrate well-log data into the inverse process in the reservoir characterization workflow, such as the integration of different sources of information in a common earth model, the estimation of the seismic distortion (also known as the wavelet filter), etc. In addition, the low vertical resolution of seismic data is an important motivation to integrate well-log information into the inverse process and thereby complement the relatively dense horizontal coverage of seismic data with high-resolution borehole data (Van Riel and Mesdag, 1988; Van Riel and Pendrel, 2000; Van Riel, 2000; Bosch et al., 2009).

Another potential benefit of seismic inversion is the ability to incorporate structural and stratigraphic information of the reservoir in order to differentiate between similar mathematical solutions on the basis of their geological viability (Van Riel and Pendrel, 2000). Cacas et al. (2008) have described a technique of stratigraphic modeling and inversion through a numerical simulations of geological processes. of the clinoforms. Specifically, their study illustrates the sensitivity of the seismic image both to subtle features of the geological model and to the parameters of the seismic modeling on the synthetic examples. Merletti and Torres-Verdin (2006) performed an experiment in which they applied deterministic and geostatistical prestack seismic inversion 
algorithms to characterize the complex progradation of a fluvio-deltaic sequence. They found that when seismic data does not permit estimation of the actual reservoir geometry, either because of its low vertical resolution or because of the high degree of facies amalgamation, the reliability of the inversion result becomes increasingly dependent on a priori models as well as on the choice of the inversion parameters. In such cases, inversion loses its ability to narrow down the likely range of solutions in model space that simultaneously honor both the seismic amplitude data and the well logs. The use of reliable a priori models in combination with a solid stratigraphic framework thus effectively decreases the degree of non-uniqueness of inversion results (Merletti and Torres-Verdin, 2006).

\subsubsection{Research Objectives}

The objective of this study is to develop a comprehensive, quantitative, inversion method which allows high-resolution characterization of fluvio-deltaic sequences. Specifically, the study focuses on fluviodeltaic clinoform systems which are known to have complex internal lithofacies distributions that are difficult to image with the seismic method. However, these sedimentary systems often contain prolific oil and gas reservoirs, and their accurate characterization is therefore important in exploration and production.

In this study two inversion methods are developed and compared with each other that differ in the way they employ stratigraphic constraints. The novelty of the first method is that the seismic data and well logs are integrated with a stratigraphic model constructed with quantitative knowledge of the reservoir architecture. The innovation of the second method lies in its goal to adopt a 'super-resolution' technique that favors sparse solutions for the clinoform characterization (Van Eekeren et al., 2008). Both inversion methods are not fully 2D, but 
more than a series of independently processed $1 \mathrm{D}$ inversions. To stress the enforced continuity along the geological structure, we use the name pseudo 2D inversion.

The potential benefits from these new approaches are that the implementation of the structural and the stratigraphic information accompanied by high vertical resolution well data to the acoustic inversion technique considerably narrows the solution space of the inverse problem and increases the confidence in the final result. The inversion methods are applied to a 3D seismic data set of an Upper Cenozoic fluvio-deltaic system in block F3 in the North Sea.

\subsection{Outline of the Thesis}

The proposed approaches integrate techniques from different disciplines, namely geophysics, geology and image analysis, and as a consequence a wide range of topics is covered in this thesis. The thesis is organized as follows: the current chapter gives an introduction to the research challenges and objectives of this thesis.

Chapter 2 presents an introduction to forward and inverse problems, followed by a theoretical background of the inversion approach and optimization techniques with respect to seismic applications.

Chapter 3 describes a 3D seismic dataset on which the methods are tested. The data set comes from the Upper Cenozoic fluvio-deltaic system of the F3 block in the North Sea. The chapter includes the geological framework, the available data, and their interpretation. Special attention is paid to the selection of the seismic data to be used for inversion tests from the total 3D volume. Since the source wavelet was not supplied with the data set, an additional section is devoted 
to the wavelet extraction from the data. In addition, for the reader's convenience, the chapter is prefaced with an overview of the structures and characteristic features of clinoform systems.

Chapter 4 presents a detailed description of the first, stratigraphic model-based, low-parametrization seismic inversion method and its application to the data set from the North Sea. The chapter is divided into three parts.

The first part describes the application of the 1D inversion for the thicknesses and acoustic impedances of the layers at the sub-seismic scale close to the well location. The second part is devoted to parameterization of the fluvio-deltaic layers of a 'system', which is the most important part of this method. The clinoform elements are geometrically modeled and estimated in the area of interest. In the third step these clinoform models guide the inversion for a comprehensive clinoform sequence characterization. The chapter ends with the presentation of the results.

The second, model-free seismic inversion method with sparsity promotion and its application on the field data set are discussed in Chapter 5. This inversion method is based on gradient operators. The objective function is modified by adding vertical and horizontal operators. A short introduction to the mathematical background is given in the beginning. Analogous to the structure of Chapter 4, first the 1D inversion application is presented for sub-seismic clinoform characterization close to the well location. The technique is then extended to a pseudo $2 \mathrm{D}$ case, followed by a discussion of the results.

Finally in Chapter 6 the advantages and limitations of both methods are discussed, followed by conclusions. Furthermore a number of recommendations for future research is given. 


\section{Chapter 2}

\section{Theoretical Background}

\subsection{The forward and inverse problem}

One of the main goals of geophysical sciences is to make inferences about physical parameters of the subsurface from the recorded data ${ }^{1}$. For a given subsurface model wave propagation theory can provide the means for computing a simulated data set; this process is known as the 'forward problem'. In exploration seismic for example, the forward model consists of a model of the subsurface, source and receiver characteristics, and the laws of physics that describe seismic wave propagation. Inverse problems apply to the opposite situation, where the aim is to reconstruct a model of the subsurface from a set of measurements. For applications in seismic exploration, the inverse problem is formulated as the problem of determining subsurface parameters, given the recorded seismic data (including the source and receiver characteristics) and the theory describing seismic wave propagation.

For seismic exploration the most straightforward and general formu-

\footnotetext{
${ }^{1}$ This section contains excerpts from two papers on geophysical inversion by Snieder (1998) and Treitel and Lines (2001).
} 


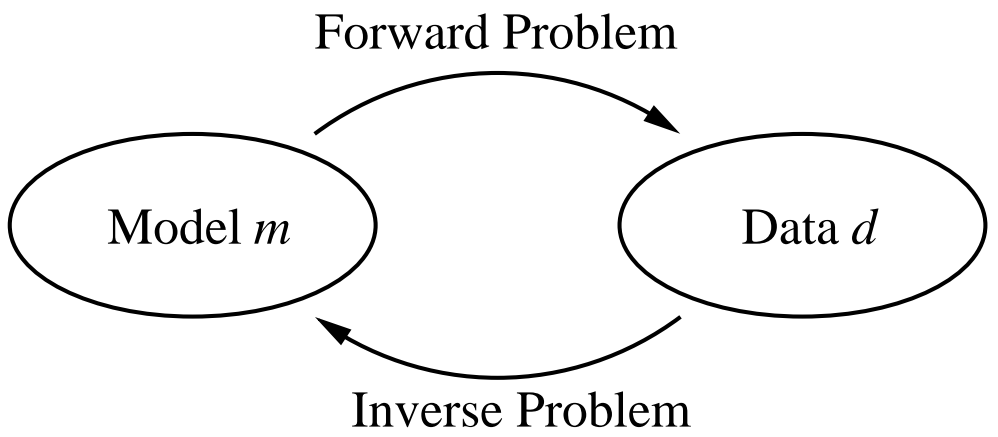

Figure 2.1: The conventional division of a problem into a forward and an inverse problem, modified from Snieder (1998).

lation leads to a full 3D non-linear inverse problem for the complete multi-offset data set. Attempts to solve this problem have been made already more than 25 years ago by Lailly (1983) and Tarantola (1984). Due to the complexity of the wave propagation theory a number of simplifying assumptions are made in the present inversion technique. Most of these simpler approaches can be formulated as parametric inverse problems (Duijndam and Drijkoningen, 1997).

In practice, we always have to deal with uncertainties and therefore an inverse problem should be formulated using probability theory. Wellknown problems in inversion are non-uniqueness, ill-posedness, and instability. Moreover, the data, source-receiver characteristics, noise models, forward model, data processing, etc., are imperfect. All maximum likelihood methods use all this information. Methods that use Bayes' rule, by contrast, employ prior information about the solution to solve the problems mentioned. 


\subsubsection{Forward modeling - 1D convolution model}

In this study we will consider the situation that the observational outcome of an experiment (seismic data) is given by a discrete data set, that is gathered in a vector $\boldsymbol{y}$. Similarly, the theory predicting the experimental outcome (the simplification of the wave propagation theory) consists of a set of functions, that are gathered in the vector function $\boldsymbol{g}(\boldsymbol{x})$, with the vector $\boldsymbol{x}$ containing the model parameters. The theory predicts the measured outcome, which is formulated in the forward model:

$$
\boldsymbol{y}=\boldsymbol{g}(\boldsymbol{x})
$$

The predicted values can not, in general, be identical to the observed values for two reasons: measurement uncertainties and modeling imperfections. These two very different sources of error generally produce uncertainties with the same order of magnitude, because, due to the continuous progress of scientific research, as soon as new experimental methods are capable of decreasing the experimental uncertainty, new theories and new models arise that allow us to account for the observations more accurately (Tarantola, 2005). Apart from the fact that the simplified version of the theory is not perfect, the measured data are always contaminated by all kinds of errors. Therefore, in common practice, a noise term $\boldsymbol{n}$ is added to the right-hand side of the equation. This yields a model that is called the standard reduced model, given by

$$
\boldsymbol{y}=\boldsymbol{g}(\boldsymbol{x})+\boldsymbol{n} .
$$

A simple model of the seismic response is known as the 1D convolution model. In this model, the subsurface is assumed to consist locally of a stack of near-horizontal, homogeneous layers (Figure 2.2). The thickness of the layers is described in terms of two-way traveltimes. 
The physical property of the layers can be defined by their acoustic impedance which, for normal incidence plane waves, is the product of the density and the compressional propagation velocity.

Ignoring transmission effects and multiple reflections, the response $s(t)$ as a function of time $t$ is the sum of reflected wavelets $w(t)$, with time delays $\tau_{j}$ and reflection coefficients $r_{j}$. Under the assumption of normal incidence of the downgoing waves on the reflectors, the reflection coefficients $r_{j}$ are determined by the acoustic impedances $Z_{j}$,

$$
r_{j}=\frac{Z_{j+1}-Z_{j}}{Z_{j+1}+Z_{j}}
$$

If the time delays are also unknown parameters to be estimated then the function $\boldsymbol{g}(\boldsymbol{x})$ becomes a nonlinear function of $\boldsymbol{x}$. The vector $\boldsymbol{g}(\boldsymbol{x})$ yields the samples of the synthetic trace $s(t)$ (Duijndam and Drijkoningen, 1997),

$$
s(t)=\sum_{j=1}^{n_{r}} r_{j} w\left(t-\tau_{j}\right),
$$

where $n_{r}$ is the number of reflectors (the interfaces separating the layers).

\subsubsection{Existence and non-uniqueness of solution}

The model that we aim to determine in geophysics, like in many inverse problems, is in general a continuous function of the space variables, having virtually infinitely many degrees of freedom. The amount of data that can be used for the determination of the model, in a realistic experiment, is by definition finite and is limited by the number of measurements. As a result, the data cannot have sufficient information to determine the model uniquely. Necessarily, bandwidth limitations 


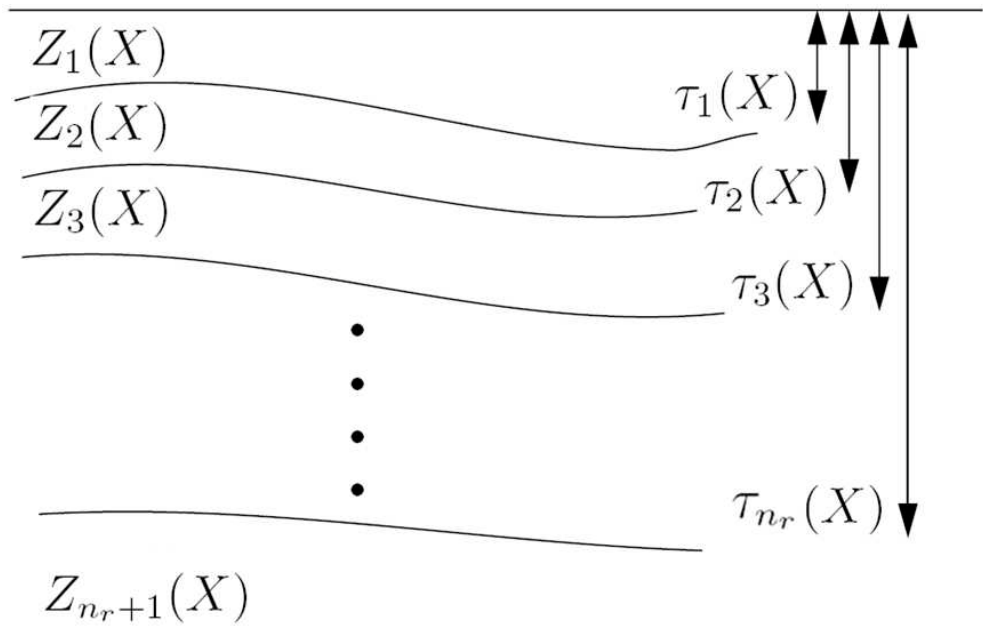

Figure 2.2: The one-dimensional structure of the subsurface. Modified from Duijndam and Drijkoningen (1997).

or other restrictions (such as a Taylor series expansion of finite order) should limit the possible number of solutions. In the modern literature this problem is known as 'finite innovation rate' of signals (Vetterli et al., 2002).

Non-uniqueness In real world experiments when a limited amount of data is available to reconstruct a model with possibly infinitely many degrees of freedom, the inverse problem can become non-unique in the sense that there are many models that explain the data equally well. A restriction on the possible outcomes should be imposed to solve the non-uniqueness (Vetterli et al., 2002).

Given measurements $\boldsymbol{y}$ and a forward model $\boldsymbol{g}(\boldsymbol{x})$ the only way of saying something about $\boldsymbol{x}$ must lie in the comparison of $\boldsymbol{y}$ with $\boldsymbol{g}(\boldsymbol{x})$. Suppose we have found two parameter vectors $\boldsymbol{x}_{1}$ and $\boldsymbol{x}_{2}$ such that 
both generate predicted data $\boldsymbol{g}\left(\boldsymbol{x}_{1}\right)=\boldsymbol{g}\left(\boldsymbol{x}_{2}\right)$ that match in some optimal sense the measured data $\boldsymbol{y}$. In this case we have no reason to prefer $\boldsymbol{x}_{1}$ to $\boldsymbol{x}_{2}$ or vice versa, so we have a non-uniqueness problem. There is not sufficient information in the combination of theory $\boldsymbol{g}(\boldsymbol{x})$ and data $\boldsymbol{y}$ to distinguish between $\boldsymbol{x}_{1}$ and $\boldsymbol{x}_{2}$. The situation may even be worse and an infinite set of parameter vectors $\boldsymbol{x}$ may yield the same optimal predicted data set $\boldsymbol{g}(\boldsymbol{x})$.

In general there are three reasons why the estimated model differs from the true model. The first reason is the discussed non-uniqueness of the inverse problem. The second one is that real data sets are always contaminated by noise. The third one is due to the simplifications in the forward and inverse modeling.

Existence The second problem of the inverse theory refers to the question of whether there is at least one model that is consistent with the data. It may seem that if the data are obtained from real measurements the existence problem is not relevant since in that case the presence of the true model (that is responsible for the recorded data) guarantees that there is at least one model that is consistent with the data. However, the facts that real data are always contaminated by noise and models are kept rather simple to keep them computationally tractable, complicates the situation.

A large discrepancy between $\boldsymbol{y}$ and $\boldsymbol{g}(\boldsymbol{x})$ may be a reason to reject $\boldsymbol{g}(\boldsymbol{x})$ and replace it by one that reduces the difference between $\boldsymbol{y}$ and $\boldsymbol{g}(\boldsymbol{x})$ alternative, if available. More often, the forward model is an approximation to the underlying laws of physics or incomplete in the sense that it does not describe all phenomena present in the data. Therefore, the inability to match modeled seismic data with measured data containing noise will not immediately lead to a falsification of the 
fundamental laws of wave propagation but rather to recognize that our modeling of the seismic experiment remains an approximation to the real experiment and model selection is seriously hampered by noise. As a result, it is common practice, in the case of noise-contaminated data to not aim to fit the data perfectly but fit it within the data errors.

From a parameter estimation point of view these are fundamental problems. In practice, these problems can be overcome by using a priori information about the parameters to be estimated. The most fundamental and straightforward method is the so-called deterministic inversion approach that employs Bayes' rule as a basis.

\subsection{Theory of seismic inversion}

In the seismic inversion technique that employs Bayes' rule, the solution of the inverse problem is obtained by integrating information concerning data, a priori information about the parameters, and theoretical relations ${ }^{2}$. Since uncertainties in information play an important role, probability theory is used for modeling. An excellent introduction to inversion and reference to seismic applications was given by Duijndam (1988); Debeye and van Riel (1999); Van Riel (2000); Bosch et al. (2009).

\subsubsection{About Bayes}

Thomas Bayes was born in a religious family in London in 1702. His father, the Rev. Joshua Bayes, was one of the first six Nonconformist ministers to be ordained in England. Thomas was also ordained a

\footnotetext{
${ }^{2}$ This section contains excerpts from two tutorials on Bayesian inversion by Ulrych et al. (2001) and Scales and Tenorio (2001) as well as from an overview paper on Bayesian inversion by Duijndam (1988).
} 
Nonconformist minister and assisted his father until he became a Presbyterian minister in the late 1720s. Thomas Bayes died in 1761 in England, misunderstood by many but on a probabilistic par with an immortal, Pierre Simon Marquis de Laplace. Bayes' theory of probability appeared posthumously in 'Essay Towards Solving a Problem in the Doctrine of Chances', published in the Philosophical Transactions of the Royal Society of London in 1764.

Bayesians versus frequentists In the Bayesian paradigm, probability distributions are the fundamental tools. Scales and Tenorio (2001) provide a good comparison of Bayesians versus frequentists. Bayesians speak of the probability of a hypothesis given some evidence and conduct pre-data and post-data inferences. Frequentists, on the other hand, are more concerned with pre-data inference and run into difficulties when trying to give post-data interpretations to their predata formulation. In other words, uncertainty estimates such as confidence sets are based on the error distribution, which is assumed to be known a priori, and on a hypothetical repetition of the data gathering process. Goutis and Casella (1995) discuss in detail the post-data inference of frequentists. The choice of prior distributions is not always well defined and in this case it would seem more reasonable to follow a frequentist approach. However, it may also be that the determinism that frequentists rely on in defining parameters may be ill-defined (Goutis and Casella, 1995). So, which approach is better? Bayesians are happy to point to some well-known inconsistencies and presumed obstacles in the frequentist methodology to use the available prior information. Some Bayesians even go as far as claiming that anyone in his/her right frame of mind should be a Bayesian. Frequentists, on the other hand, complain about the sometimes subjective choice of priors and about the computational complexity of the Bayesian approach. For colorful discussions on the comparison of the two approaches, see 
Efron (1986) and Lindley (1975). Also see Rubin (1984) for ways in which frequentist methods can complement Bayesian inferences.

\subsubsection{Bayes' rule as a basis for inverse problems}

When we try to describe an observation with a mathematical model, it will often contain free parameters whose values have to be estimated. These parameters can be gathered in the vector $\boldsymbol{x}$ and the vector $\boldsymbol{y}$ contains a discretized data vector. Suppose $p(\boldsymbol{x}, \boldsymbol{y})$ reflects the state of information on $\boldsymbol{x}$ and $\boldsymbol{y}$ before measurements for $\boldsymbol{y}$ are obtained. When data as a result of measurements determine the values for $\boldsymbol{y}$ then the state of information on $\boldsymbol{x}$ should be represented by $p(\boldsymbol{x} \mid \boldsymbol{y})$, which is given by Bayes' rule:

$$
p(\boldsymbol{x} \mid \boldsymbol{y})=\frac{p(\boldsymbol{y} \mid \boldsymbol{x}) p(\boldsymbol{x})}{p(\boldsymbol{y})} .
$$

Here, $p(\boldsymbol{x} \mid \boldsymbol{y})$ is the so-called a posteriori probability, $p(\boldsymbol{y} \mid \boldsymbol{x})$ the likelihood function for observing $\boldsymbol{y}$ given the model parameters $\boldsymbol{x}, p(\boldsymbol{x})$ the priori probability of the model parameters and $p(\boldsymbol{y})$ a constant scaling factor that does not depend on $\boldsymbol{x}$. It is important to realize that $p(\boldsymbol{x} \mid \boldsymbol{y})$ contains all information available on $\boldsymbol{x}$ given the data $\boldsymbol{y}$ and therefore is in fact the solution to the inverse problem.

\subsubsection{The likelihood function}

The likelihood function $p(\boldsymbol{y} \mid \boldsymbol{x})$ gives the probability of the data, given the parameters $\boldsymbol{x}$. Most inverse problems can be treated using the standard reduced model (Bard, 1973):

$$
\boldsymbol{y}=\boldsymbol{g}(\boldsymbol{x})+\boldsymbol{n},
$$

where $\boldsymbol{g}(\boldsymbol{x})$ is the forward model, which can be nonlinear. The vector $\boldsymbol{n}$ contains the errors or the noise. Duijndam (1988) has shown 
that the approach of Tarantola and Valette (1982a,b), which distinguishes theoretical and observational errors on a more fundamental level, yields the same result as the Bayesian approach under different interpretations of theoretical and observational errors, suggesting that this distinction is arbitrary. Therefore, the vector $\boldsymbol{n}$ represents the errors and noise. For a more detailed discussion about the different noise components impact on the inversion results see Tarantola (2005). In case the noise can be assumed to be Gaussian with zero mean and covariance matrix $\boldsymbol{C}_{n}$ the likelihood function is:

$$
p(\boldsymbol{y} \mid \boldsymbol{x})=\frac{1}{(2 \pi)^{m / 2}\left|\boldsymbol{C}_{n}\right|^{1 / 2}} \exp \left\{-\frac{1}{2}(\boldsymbol{y}-\boldsymbol{g}(\boldsymbol{x}))^{T} \boldsymbol{C}_{n}^{-1}(\boldsymbol{y}-\boldsymbol{g}(\boldsymbol{x}))\right\},
$$

where $m$ is the number of data points.

\subsubsection{A priori information}

Any type of information about the parameters that is available independently of the data can be used as a priori information and is represented by $p(\boldsymbol{x})$. A priori knowledge about parameters can be divided in two terms: an idea about the values, but also the uncertainties in these values. A Gaussian probability density function is the most convenient way to describe this type of information:

$$
p(\boldsymbol{x})=\frac{1}{(2 \pi)^{n / 2}\left|\boldsymbol{C}_{x}\right|^{1 / 2}} \exp \left\{-\frac{1}{2}\left(\boldsymbol{x}-\boldsymbol{x}_{i}\right)^{T} \boldsymbol{C}_{x}^{-1}\left(\boldsymbol{x}-\boldsymbol{x}_{i}\right)\right\}
$$

where $n$ is the number of parameters, $\boldsymbol{x}_{i}$ is the mean of the distribution and $\boldsymbol{C}_{x}$ is the covariance matrix, which specifies the uncertainties. 


\subsubsection{Uncertainties}

The covariance matrix $\boldsymbol{C}$ is defined by its elements:

$$
\boldsymbol{C}=E(\boldsymbol{x}-\boldsymbol{\mu})(\boldsymbol{x}-\boldsymbol{\mu})^{T}=\int(\boldsymbol{x}-\boldsymbol{\mu})(\boldsymbol{x}-\boldsymbol{\mu})^{T} p(\boldsymbol{x}) \mathrm{d} \boldsymbol{x},
$$

where $\boldsymbol{\mu}$ is the vector with expected values of $\boldsymbol{x}$ (e.g., the priors or mean) and superscript $T$ denotes the transpose operator. The diagonal elements of $\boldsymbol{C}$ are the variances $\sigma_{i}^{2}=E\left(x_{i}-\mu_{i}\right)^{2}$ of the variables $x_{i}$. In the specific case that $\boldsymbol{y}$ is the time-migrated poststack seismic data and a $1 \mathrm{D}$ forward model is used in the inversion, the covariance matrix of the noise $\boldsymbol{C}_{n}$ contains temporal as well as spatial band-limitations. When the latter are neglected, $\boldsymbol{C}_{n}$ has a diagonal block structure containing blocks $\boldsymbol{C}_{n_{k}}$. Each block is the covariance matrix for an individual seismic trace. The number of blocks are defined by the number of seismic traces $k$ in $\boldsymbol{y}$. Under the assumption of white Gaussian noise each individual covariance matrix $\boldsymbol{C}_{n_{k}}$ is defined by:

$$
\boldsymbol{C}_{n_{k}}=\operatorname{diag}\left\{\sigma_{n_{k}}^{2}\right\},
$$

where $\sigma_{n_{k}}$ is the variance of the $k^{\text {th }}$ trace. If the a priori information for the various parameters is uncorrelated, the covariance matrix of parameter uncertainties is a diagonal matrix:

$$
\boldsymbol{C}_{x}=\operatorname{diag}\left\{\sigma_{x}^{2}\right\},
$$

where $\sigma_{x}$ is the vector of uncertainty variances.

\subsubsection{Point Estimation}

The inspection of the a posteriori probability density function (pdf) through the whole of parameter space is impractical, if not impossible. Therefore, a so-called point estimate can be computed. To estimate 
the parameters in a least-squares sense, the mean of $p(\boldsymbol{x} \mid \boldsymbol{y})$ is obtained (Bard, 1973):

$$
\hat{\boldsymbol{x}}=\int \boldsymbol{x} p(\boldsymbol{x} \mid \boldsymbol{y}) \mathrm{d} \boldsymbol{x} .
$$

This estimator $\hat{\boldsymbol{x}}$ is called the least-mean-squared error, or the Bayes estimator. The evaluation of equation (2.12) requires the computation of $p(\boldsymbol{x} \mid \boldsymbol{y})$ through the whole parameter space. In most cases, this is practically impossible. Therefore, an alternative and more practical solution is to choose the maximum of the a posteriori density function, a value also referred to as the MAP estimation. When $p(\boldsymbol{x} \mid \boldsymbol{y})$ is symmetrical and unimodal, the mean coincides with the mode and the least-means-squared estimator is equivalent to the MAP estimator. This estimator provides the most likely values of the parameters given data and a priori information. For a uniform, a priori distribution $p(\boldsymbol{x})$, which is often taken as the state of null information, it is easily seen that the maximum of the a posteriori density function coincides with the maximum of the likelihood function. The MAP estimation is then equivalent to the maximum likelihood estimation (MLE). For more information about the asymptotic properties of the MAP estimation and the MLE, we refer to Bard (1973).

\subsubsection{Nonlinear least-squares estimation}

Analytical results of the MAP estimation depend on the form of the probability density functions involved. Assuming Gaussian distributions for the noise and the a priori information, we find that maximizing the product of $p(\boldsymbol{y} \mid \boldsymbol{x})$ and $p(\boldsymbol{x})$ is equivalent to maximizing the sum of the exponents or minimizing the function $F(\boldsymbol{x})$, defined by:

$$
F(\boldsymbol{x})=(\boldsymbol{y}-\boldsymbol{g}(\boldsymbol{x}))^{T} \boldsymbol{C}_{n}^{-1}(\boldsymbol{y}-\boldsymbol{g}(\boldsymbol{x}))+\left(\boldsymbol{x}-\boldsymbol{x}_{i}\right)^{T} \boldsymbol{C}_{x}^{-1}\left(\boldsymbol{x}-\boldsymbol{x}_{i}\right) .
$$


The first term is the so-called data mismatch and is the weighted $L_{2^{-}}$ norm of the residuals $\boldsymbol{y}-\boldsymbol{g}(\boldsymbol{x})$. The second term is a weighted $L_{2}$-norm of the deviations of the parameters from their a priori mean values $\boldsymbol{x}_{i}$. From a non-Bayesian point of view this term stabilizes the solution. It is not present in maximum likelihood estimation. The relative importance of data mismatch and parameter deviations is determined by their uncertainties as specified in $\boldsymbol{C}_{n}$ and $\boldsymbol{C}_{x}$. The model $\boldsymbol{x}$ that minimizes $F(\boldsymbol{x})$ should make $\boldsymbol{g}(\boldsymbol{x})$ close to $\boldsymbol{y}$ and at the same time stay close to the a priori model $\boldsymbol{x}_{i}$.

\subsubsection{The probability density function selection}

The important issue to consider is which type of pdf is to be used for the noise and the a priori information. It has been shown by Bard (1973) that the Gaussian probability density function has some advantages, namely:

1. The distribution of the sum of a large number of identically distributed independent random variables is Gaussian (central limit theorem).

2. The Gaussian probability density function is able to approximate the distribution of many repeated measurements in nature.

3. Given the mean and the covariance, the Gaussian probability density function has the highest information content as determined by Shannon's information measure, which is defined by Shannon (1948), for a dimensionless pdf:

$$
I=E(\log p(\boldsymbol{x}))=\int p(\boldsymbol{x}) \log p(\boldsymbol{x}) \mathrm{d} \boldsymbol{x},
$$


This shows that once the mean and the covariance are known, we do not use more information than we legitimately know by choosing the Gaussian pdf.

4. The Gaussian probability density function is mathematically as well as computationally most convenient.

The first point refers to the data only. Point two may also apply to a priori information, when information from several sources is combined. The third point is a strong argument in favor of Gaussian pdf's, because Shannon's information measure has some attractive advantages one would want to demand of such a measure. Of course, crucial questions arise whether or not a covariance matrix is available in practice. Suppose an expert working on the problem has an idea about the uncertainty of a priori information but it is still questionable whether the uncertainty value is to be attributed to a standard deviation. Although standard deviations are often used to indicate uncertainties in practice, this usage must be based on the (implicit or explicit) assumption that the underlying pdf has a form close to the Gaussian one. For this pdf, the standard deviation is quite a reasonable measure of uncertainty with the interval of $(-\sigma, \sigma)$ corresponding to a $67 \%$ confidence interval. The fourth point can be regarded as the most important argument for us to use Gaussian pdf's, since it is very convenient from a mathematical point of view, as well as because a lot of fast optimization schemes have been introduced for the resulting least-squares problems.

The issue concerning the type of pdf for the noise present in the data generates continuous interest. This issue represents a way of thinking that is typical for an objective interpretation of the concepts of probability. In this interpretation a number of known (in the sense of identified) or unknown processes constitutes a random generator corrupting the data. There were some ideas proposed to try to find the 
pdf according to which the errors are generated. In reality however, the dimension of the pdf is equal to the number of data points. Since there is only one realization available, the pdf from it can never be determined. Therefore, the assumption of repetitiveness is needed in order to get the noise samples identically distributed, in order to be able to say something about the form of the pdf. This assumption however, can never be tested for its validity and is therefore metaphysical rather than physical. It was proposed to follow another way of reasoning in the subjective Bayesian interpretation (Barnett, 1982). If the noise reflects our uncertainties concerning the combination of data and theory, then the pdf should simply be selected such that our knowledge is represented as accurately as possible. Because it is not a trivial step by itself, the inspection of residuals after inversion may give reason to modify the model or the parameters of the distribution chosen.

\subsection{Regularization}

In general inverse problems there are two positive functionals, say $A$ and $B$. The first, $A$, measures something like the agreement of a model to the data, or, a related quantity like the 'sharpness' of the mapping between the solution and the underlying data. When $A$ by itself is minimized, the agreement or sharpness may become very good (often impossibly good), but the solution becomes unstable, wildly oscillating, or in other ways unrealistic, reflecting that the first term alone typically defines a highly degenerated minimization problem. That is where the second term $B$ comes in. It measures something like the 'smoothness' of the desired solution, or a related quantity, that parametrizes the stability of the solution with respect to variations in the data, or a quantity reflecting a priori information about the probability of a solution. $B$ is called the stabilizing functional or reg- 
ularization operator and its quantities may reflect a priori information regarding the solution found. In any case, minimizing the second term (by itself) is supposed to give a solution that is 'smooth' or 'stable' or 'likely' - and that has nothing to do with the measured data (Press et al., 2007). The single central idea in inverse theory is the prescription:

$$
\min _{\boldsymbol{x}}\left\{A(\boldsymbol{y}, \boldsymbol{g}(\boldsymbol{x}))+\lambda B\left(\boldsymbol{x}_{i}, \boldsymbol{x}\right)\right\} .
$$

Varying $\lambda$ for positive values yields the so-called trade-off curve by plotting values of $A$ versus $B$ at the optimal point of equation (2.15) and parametrized by $\lambda$ (see Figure 2.3). From these observations one must find the 'best' value for $\lambda$ according to some criterion, ranging from fairly objective to entirely subjective (Press et al., 2007). Equation (2.15) has a natural Bayesian interpretation.

Perhaps the most common regularization scheme is the Tikhonov regularization. A weighted sum of $A$ and $B$ is formed using a weighting factor $\lambda$ and then the image $x_{\lambda}$ which minimizes this sum is found,

$$
x_{\lambda}=\operatorname{argmin}\left\{\|A\|^{2}+\lambda\|B\|^{2}\right\} .
$$

The regularizing parameter $\lambda$ can be thought of as controlling the balance between minimizing the data misfit term and the regularizing term. When $\lambda$ is small, there is little weight put on the regularizing term, the data is described well and the solution is in general not sparse. Conversely, when $\lambda$ is large, the regularizer dominates the minimization and the reconstructed image is smooth at the expense of not fitting the data so well.

In the previously described method of Duijndam (1988) the relative importance of data mismatch and parameter deviations is determined by their uncertainties as specified by $\boldsymbol{C}_{x}$ and $\boldsymbol{C}_{n}$. This only holds when the estimation of the parameters' uncertainties can be done quite 


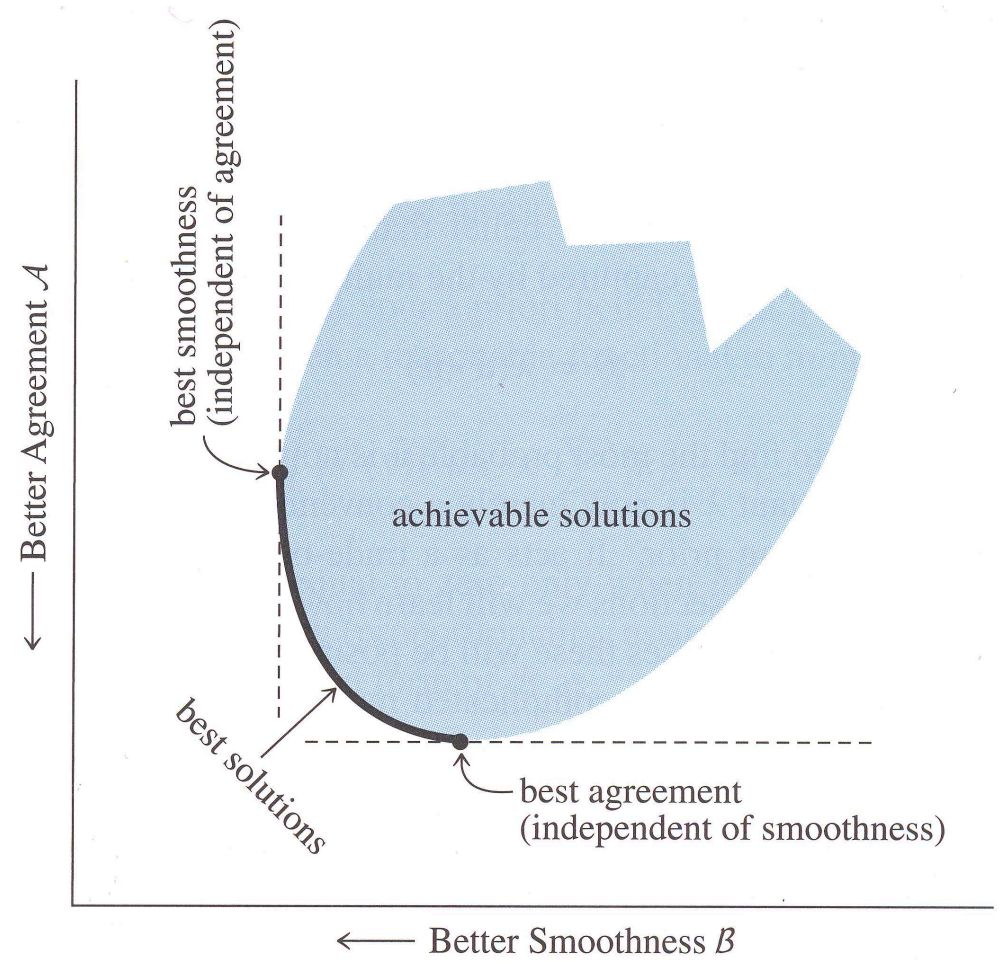

Figure 2.3: Almost all inverse problem methods involve a trade-off between two optimizations: agreement between data and solution, or 'sharpness' of mapping between true and estimated solutions (here denoted as $A$ ), and smoothness or stability of the solution (here denoted as $B$ ). Among all possible solutions, shown here schematically as the shaded region, those on the boundary connecting the unconstrained minimum of $A$ and the unconstrained minimum of $B$ are the 'best' solutions, in the sense that every other solution is dominated by at least one solution on the curve (Press et al., 2007).

accurately, otherwise some additional regularization is required. For 
such a case we can replace equation (2.13) by

$$
F(\boldsymbol{x})=(\boldsymbol{y}-\boldsymbol{g}(\boldsymbol{x}))^{T} \boldsymbol{C}_{n}^{-1}(\boldsymbol{y}-\boldsymbol{g}(\boldsymbol{x}))+\lambda\left(\boldsymbol{x}-\boldsymbol{x}_{i}\right)^{T} \boldsymbol{C}_{x}^{-1}\left(\boldsymbol{x}-\boldsymbol{x}_{i}\right) .
$$

The big question now is how to choose $\lambda$. For a linear inverse problem, perhaps the most convenient graphical tool for setting $\lambda$ is the well-known 'L-curve' method. When we plot $\log \|\boldsymbol{y}-\boldsymbol{g}(\boldsymbol{x})\|_{2}^{2}$ versus $\log \left\|\boldsymbol{x}-\boldsymbol{x}_{i}\right\|_{2}^{2}$ we often get a characteristic L-shaped curve with a distinct corner separating the vertical and horizontal part of the curve (Figure 2.4).

The rationale for using the L-curve is that regularization is a trade-off between the data misfit and a penalty for solutions that violate the prior knowledge we may have regarding the solution. In the vertical part of the curve, the $\left(\boldsymbol{x}-\boldsymbol{x}_{i}\right)$ term is a very sensitive function of the regularization parameters, because the solution is undergoing large changes with $\lambda$ in the attempt to fit the data better. On the horizontal part of the curve, the solution is not changing much as $\lambda$ is changed. However, the data misfit is increasing sharply with more filtering. It is desired to choose a solution which lies not too far to the right of the corner (Figure 2.4). Here, both terms have approximately the same importance, with a small preference for fitting the measurements.

\subsection{Optimization}

The measured and the theoretical geophysical responses are matched by the use of a suitable optimization algorithm. All such algorithms are designed to minimize the functional $F(\boldsymbol{x})$ like defined in equation (2.17). It is typical for many schemes to start out with an initial guess of the model parameters, from which an initial model response can be computed. As a second step, the optimization algorithm yields a set 


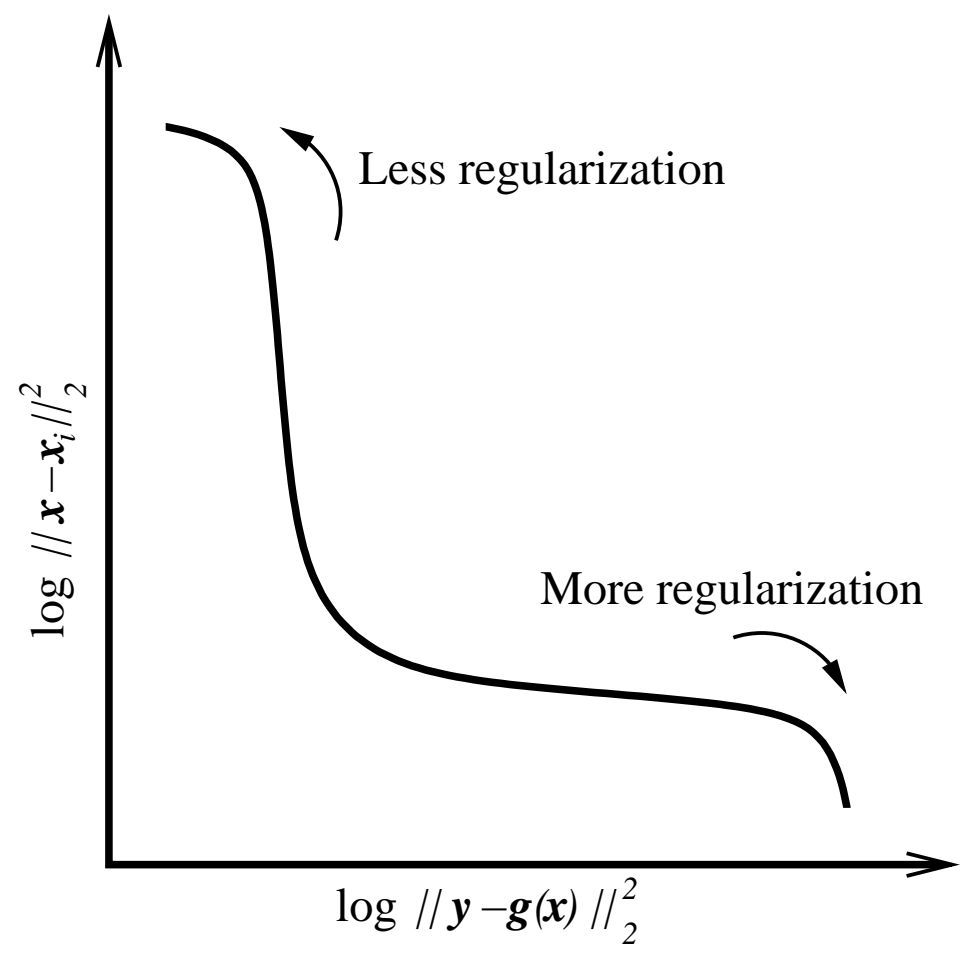

Figure 2.4: The generic form of the L-curve (Tan and Fox, 1996).

of updated parameter estimates, which are afterwards used as input to the theoretical model, and the resulting new theoretical response produces an improved match to the real data. If this happens, the inversion is said to converge; if not, there are numerous alternative means to achieve convergence, although none of the existing methods always works. Due to the non-linearity of the model response as a function of the model parameters, it is necessary to do these calculations iteratively, that is, the above procedure must be applied many times in succession until a satisfactory degree of agreement between 
the theoretical and the recorded seismic responses has been achieved.

Although, in principle, any optimization technique that finds the minimum will do, in practice efficiency considerations usually make the proper choice of an optimization algorithm a very important one. Many textbooks have appeared on the subject of (local) optimization, for example Gill et al. (1982); Scales (1985), or more recently Tarantola (2004) and Griva et al. (2009).

Finding the minimum of the penalty function is an art in itself. In general, one either uses a (steepest) descent method where one iteratively updates an estimate of the model that minimizes the penalty function by moving 'downhill' in one way or another, or one employs techniques that sample the model space in many different locations as a way of minimization. Descent methods can relatively easily be implemented for large-scale inverse problems. The main drawback of this approach is that for nonlinear inverse problems the penalty function may have several minima (Figure 2.5). A descent method may lead to a model estimate that corresponds to a local estimate of the penalty function rather than the global minimum.

\subsubsection{Simplex method}

The simplex method is a direct search method that does not use numerical or analytic gradients. The methodology of the simplex method is given in Nelder and Mead (1965), with a discussion given in Gill et al. (1982) and Woods (1985). It works on the principle of evaluating the merit function on a set of $M+1$ points called an $M$ simplex. A simplex is the simplest entity which encloses a 'volume' in $M$ dimensions, and the idea is to try to enclose the position of the minimum of the merit function within the volume of the simplex. By applying a series of transformations based on the function values at the vertices, the 


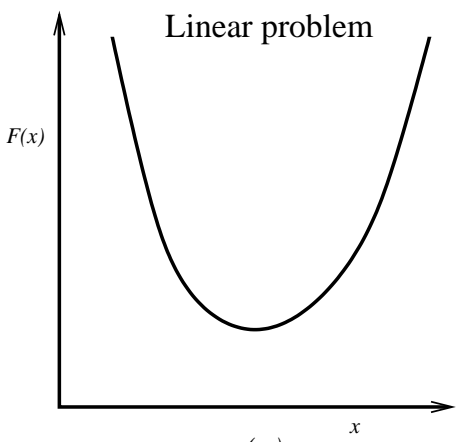

(a)

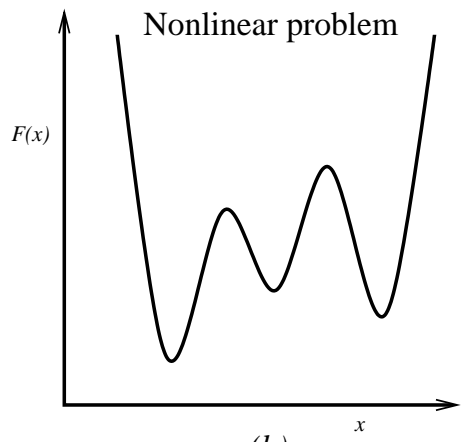

(b)

Figure 2.5: (a) The least-squares misfit function for a linear problem. (b) The conventional view of the misfit function for a nonlinear inverse problem (Snieder, 1998).

simplex moves downhill and shrinks until it is small enough to specify the minimum position to the desired accuracy. The main advantages of the simplex method are its simplicity, in that it only requires function evaluations, and its robustness to non-smooth merit functions. This method uses only function values (no derivative values), and is especially suitable for noisy functions. The disadvantage is its often prohibitively slow speed when $M$ is moderate or large (Tan and Fox, 1996).

\subsubsection{Gradient-based method}

In the Newton optimization methods both first and second order derivatives of the cost function to be minimized are used. Derivatives should be given whenever possible, as suggested by Scales (1985). When they are not available they can be generated with finite difference techniques. For second order derivatives, however, this may be too expensive and attractive alternatives are the quasi-Newton methods. In 
these methods, the Hessian matrix or its inverse is approximated at each iteration, using an updating formula that uses the changes in the gradient vector at subsequent iterations. Under the assumption of Gaussian distributions for noise and a priori information, the process of finding the maximum of the a posteriori probability leads to a (nonlinear) least-squares inverse problem. Special quasi-Newton methods like Levenberg-Marquardt (Moré, 1978) can be devised when the function to be minimized is a sum of squares, which defines the maximum of the a posteriori probability under Gaussian assumptions. The cost function in the framework of the Levenberg-Marquardt method has a format like $\min _{\beta} \sum_{i}^{N}\left(\chi-\chi_{i}(\beta)\right)^{2}$, where $\chi$ is the measurement and $\chi_{i}(\beta)$ is the estimate depending on parameter $\beta$.

The (general) nonlinear problem is typically solved by an iterative application of a given optimization algorithm. The problem is that in order for convergence to the 'correct' subsurface model to take place, the initial guess must be 'close' to the true solution. More generally, there is a trade-off between resolution and noise suppression: a better resolved (sharper) solution is achievable only at the price of poorer noise suppression, and vice-versa. To put this yet in another way, we always face a trade-off between a solution's resolving power and its ability to describe the observed data. 


\section{Chapter 3}

\section{Field Data Set}

Fluvio-deltaic sedimentary systems are of great interest for explorationists because they constitute potential hydrocarbon reservoirs. However these systems are among the most complex and heterogeneous ones encountered in the subsurface. Geological bodies of interest can have dimensions close to, or below the resolution of the seismic dataset, which means only some of them are recognizable in a seismic image.

\subsection{Clinoform Systems}

Stratigraphic signatures and stratal patterns in the sedimentary rock record are a result of the interaction of tectonics, eustasy and climate. Tectonics and eustasy control the amount of space available for the sediment to accumulate (accommodation), and tectonics, eustasy and climate interact to control sediment supply and how much of the accommodation is filled ${ }^{1}$. Clinoforms systems can result from these

\footnotetext{
${ }^{1}$ This section contains excerpts from a book on sequence stratigraphy by Emery and Myers (2005).
} 
processes; they are a common feature in a basin-margin setting and commonly have relatively flat topsets, sloping foresets (often termed simply clinoforms) and relatively flat bottomsets (Figure 3.1).

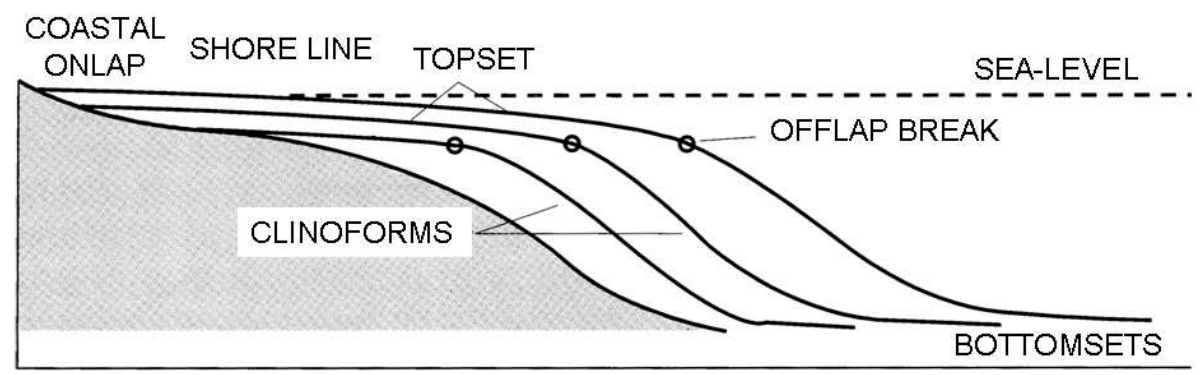

Figure 3.1: Typical profile of a prograding basin-margin unit, comprising topsets and clinoforms separated by a break in slope, the offlap and bottom sets. Modified from Emery and Myers (2005).

Topsets are found at the proximal portion of the basin-margin profile and are characterized by low gradients $(<0.1$ degree). They often effectively appear flat on seismic data and generally contain alluvial, deltaic and shallow-marine deposits. The main break in slope in the depositional profile occurs between the topset and the clinoform and is called the offlap break. The shoreline can be located at any point within the topset; it can coincide with the offlap break or may occur hundreds of kilometers landward. The clinoform forms the steeper dipping portion of the basin-margin profile ( $>1$ degree) located basinward of the topset. Clinoforms generally contain deeper water deposits characteristic of the slope. The slope of the clinoform generally can be easily seen on seismic data.

The bottom set describes the portion of the basin-margin profile at the base of the clinoform characterized by low gradients and containing deep-water deposits. 
The profile of a clinoform system results from the interplay between sediment supply and wave, storm and tidal energy in the basin. Sediment enters the proximal end of the profile through river systems and is distributed across the topset area by wave- and/or current-related processes. These may include fluvial currents, tidal currents, storm currents, etc. However, the topset transport processes are effective only at relatively shallow depths of up to few tens of meters. At its edge, a slope develops that allows sediment transportation by gravity process into deeper water. The clinoforms build up to an angle needed to transport sediment downslope. This is strongly influenced by sediment size: coarse-grained sediment, with a higher angle of repose, will build up steeper slopes than fine-grained sediment (Kenter, 1990).

\subsubsection{Basin architecture}

The rate of sediment supply controls how much and where accommodation is filled. The balance between sediment supply and relative sea-level rise controls whether facies belts prograde basinwards or retrograde landwards. In order to understand the behavior of a topset/clinoform margin through time it is necessary to consider the balance between the rate of sediment supply and the rate of creation of topset accommodation volume. The rate of change of accommodation volume is a function of the magnitude of the sea-level rise multiplied by the topset area (Milton and Bertram, 1995). If, during the same time interval, the basin margin is supplied with a greater volume of sediments, then the topset accommodation volume will be completely filled, and sediment will deposit on the clinoforms causing the offlap break to prograde basinwards (Figure 3.2)

Progradational geometries therefore occur when sediment supply exceeds the rate of creation of topset accommodation volume and facies belts migrate basinwards. On seismic data progradation is expressed 


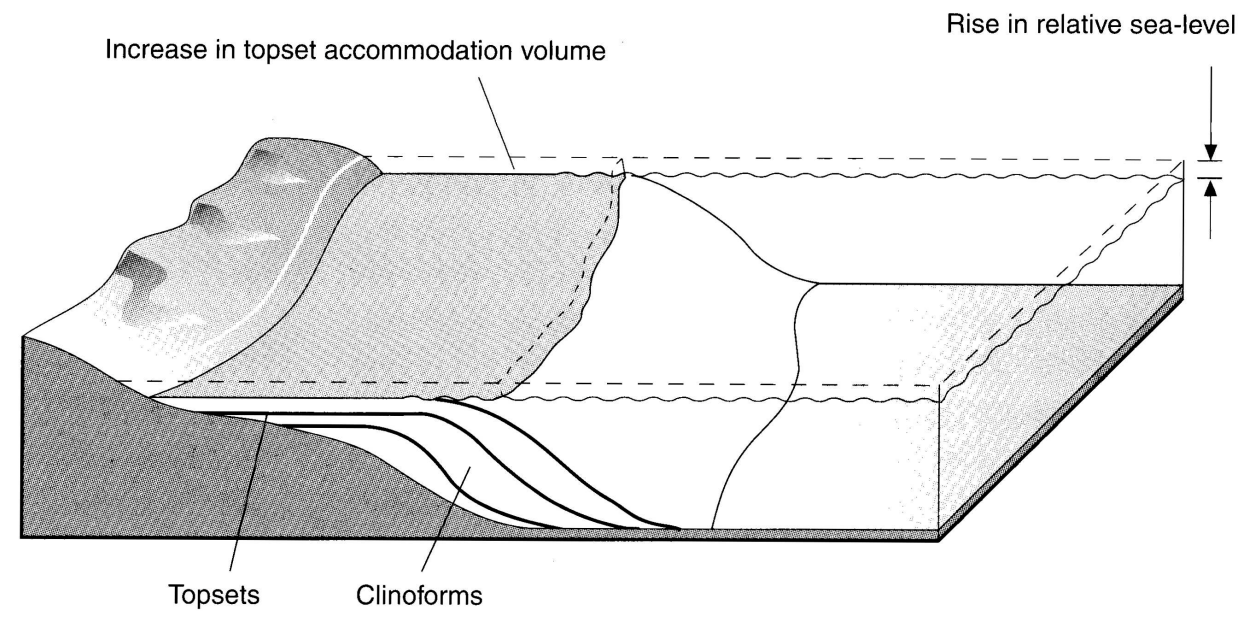

Figure 3.2: The increment of topset accommodation volume caused by a rise in relative sea-level is equal to the product of the rise in relative sea-level and the topset area. Modified from Emery and Myers (2005).

as clinoforms that show a basinwards migration of the offlap break. Regression is a term that is used to refer specifically to a basinwards movement of the shoreline. Aggradational geometries occur when sediment supply and rate of creation of topset accommodation volume are roughly balanced. Facies belts stack vertically and the offlap break does not migrate landward or basinward. Retrogradational geometries occur when sediment supply is less then the rate of creation of topset accommodation volume. Facies belts migrate landward and the former depositional offlap break becomes a relict feature. Transgression is a term used to refer specifically to the landward movement of the shoreline. These different types of clinoform profiles are shown in Figure 3.3. 


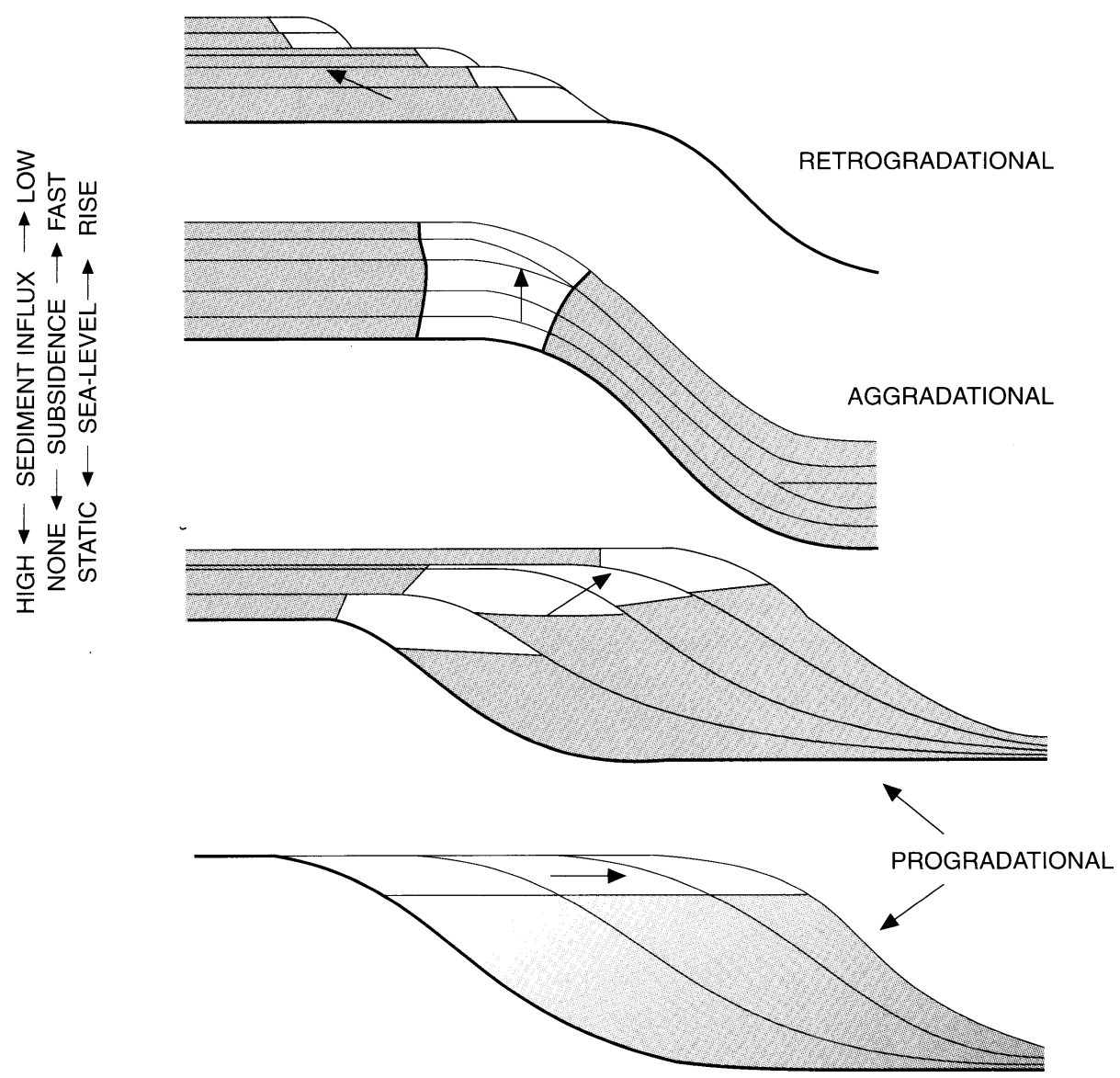

Figure 3.3: Depositional architecture as a function of accommodation volume and sediment supply (Emery and Myers, 2005).

\subsubsection{The log response in clinoform systems}

Trends in log response at any scale reflect trends in depositional energy, and thus with patterns of sedimentary infill. Typical log trends are 
illustrated in Figure 3.4.

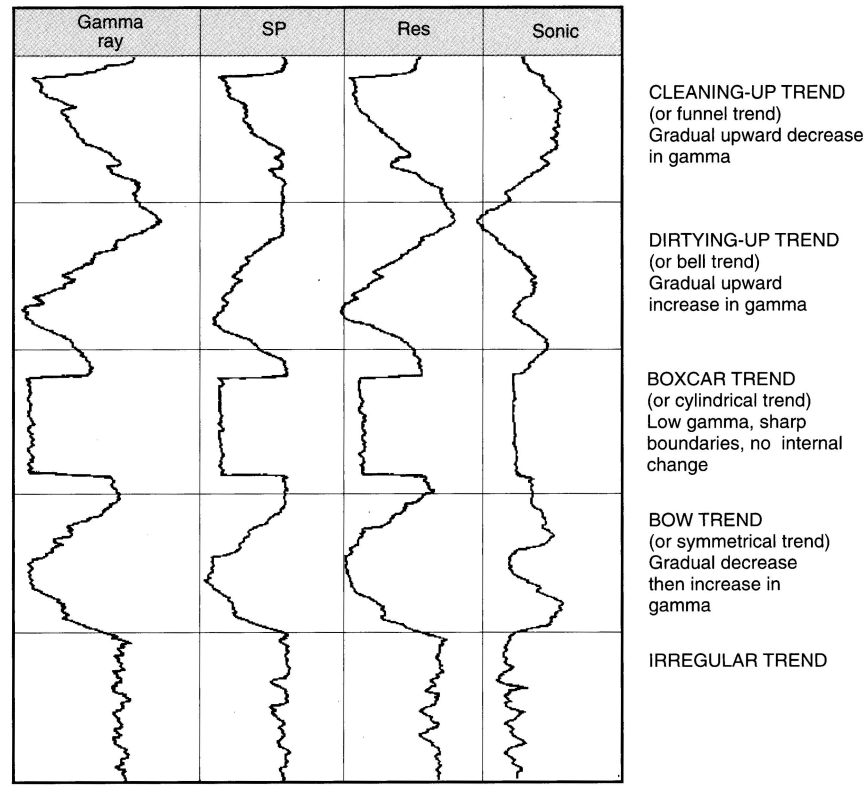

Figure 3.4: Idealized log trends, assuming saltwater-filled porosity. The sonic assumes no cementation. Porous sands are slower than shales in this example (Emery and Myers, 2005).

The cleaning-up trend shows a progressive upward decrease in the gamma ray reading, representing a gradual upward decrease in clay content. In shallow marine settings the cleaning-up motif is usually related to an upward transition from shale-rich to sand-rich lithologies, owing to an upward increase in depositional energy, upward shallowing and upward coarsening.

The dirtying-up trend shows a progressive upward increase in the gamma ray reading related to a gradual upward increase in the clay- 
content. This could be a lithology change, for example from sand to shale, or an upward-thinning of sand beds in a thinly interbedded sand-shale sequence. Both imply a decrease in depositional energy. In shallow marine settings the dirtying-up trend often reflects the retreat or abandonment of shoreline-shelf systems, resulting in upward deepening and a decrease in depositional energy.

Boxcar log trends (also known as cylindrical motifs) are sharp-based low-gamma ray units with an internally relatively constant gamma ray reading, set within a higher gamma ray background. The boundaries with the overlying and underlying shales are abrupt. The sonic reading from the sand may be either a higher or a lower transit time than in the shales, depending on cementation and compaction.

The bow trend (also known as barrel trend or symmetrical trend) consists of a cleaning-up, overlain by dirtying-up trend of similar thickness and with no sharp break between the two. It is generally the result of a waxing and waning of clastic sedimentation rate in a basinal settings, where the sedimentation is unconstrained by base level.

Irregular trends have no systematic change in either base line, and lack the clean character of the boxcar trend. They represent aggradation of a shaley or silty lithology, and may be typical of shelfal or deep water settings, a lacustrine succession, or muddy alluvial overbank facies.

The log response of a system tract thus can vary significantly, depending on where the well passes through the clinoform system. A clinoform unit can be inferred from a cleaning-upwards pattern that reflects upward shallowing. The base of the cleaning-up pattern can be equivalent to a downlap surface. Confirmation that this log response represents a prograding clinoform pattern could come from core or biostratigraphic data supporting an upward-shallowing trend, because 
in clastic systems this can occur only through progradation, except in special circumstances. Additional confirmation is best sought from seismic data, with subsidiary data from dipmeter studies, and the inferred setting within the basin and the stratigraphy (e.g., between basinal facies below and basin-margin facies above).

The base of a clinoform unit is a downlap horizon. This may be recognized as a distinct base to the cleaning-up unit, often with log facies diagnostic of marine condensation, such as a high-gamma ray shale, or a cemented horizon. In other cases the downlap surface is more difficult to identify. The top of a cleaning-up clinoform trend may be marked either by an abrupt increase in shale content (gamma ray reading), resulting from abrupt deepening across the transgressive surface, or it may be overlain by topsets. The log responses of basinal units tend to be more symmetrical than the log response of clinoforms or topsets. The exact nature of the log response depends on the nature of the sediments; mud-rich basinal units tend to show a symmetrical bow response, whereas sand-prone systems tend to show a box-car or cylindrical log trend. In units thick enough to be resolvable on seismic data, breaks in the cleaning-up log profile generally tie with breaks within the clinoforms. An abrupt increase in shale content within the clinoform trend implies an abrupt upward jump to a deeper facies, resulting from lobe switching or transgression during relative sea-level rise. Similarly an abrupt decrease in the gamma ray response may imply an abrupt jump to a shallower facies, and thus a sequence boundary, a normal fault or a slump. The thickness of the clinoform interval on the logs gives an approximate measure of the clinoform height, and thus the basin water depth (modified by compaction, the effect of syn-depositional subsidence and other factors).

Topset units are formed by repeated cycles of filling of accommodation space between the offlap break and the coastal onlap point, and they 
are seen as small-scale cycles on logs. Most common is the cleaning-up motif, widely recognized in parasequences from marine settings. In the cleaning-up motif the shale content decreases upwards, whereas primary porosity and bed thickness (as determined from microresistivity logs) may increase upwards. On the gamma ray log the parasequences are small-scale cleaning-up units, and the marine flooding surfaces are abrupt upwards increases in gamma reading. Progradation, aggradation and retrogradation of the basin-margin system may be recognizable from the way units are stacked into sets.

\subsection{Geological Framework}

The data set used in this study is from the F3 block in the Dutch sector of the Southern North Sea. The entire North Sea region in the Cenozoic era (Figure 3.5) is characterized by a thermally subsiding epicontinental basin that was confined by land-masses apart from a narrow seaway connecting the Norwegian-Greenland Sea in the North ${ }^{2}$. At the end of the early Paleocene mainly chalky sediments were deposited in the region, but following it a sudden increase in supply of siliciclastics occurred because of thermal uplift of the British Isles and the compression of the central European Alpine foreland (Laramide tectonic phase). Hence, the deposition of chalky sediments ceased (Ziegler, 1990).

The Ringkobing-Fyn High had acted as a structural high since Permian times and influenced the depositional patterns in the Cenozoic (Figure 3.6). It separates the central and the southeastern North Sea into two parts as the northern and the southern embayments respectively.

\footnotetext{
${ }^{2}$ This section contains excerpts from two papers on Cenozoic deposits in the North Sea by Sørensen et al. (1997) and Steeghs et al. (2000).
} 


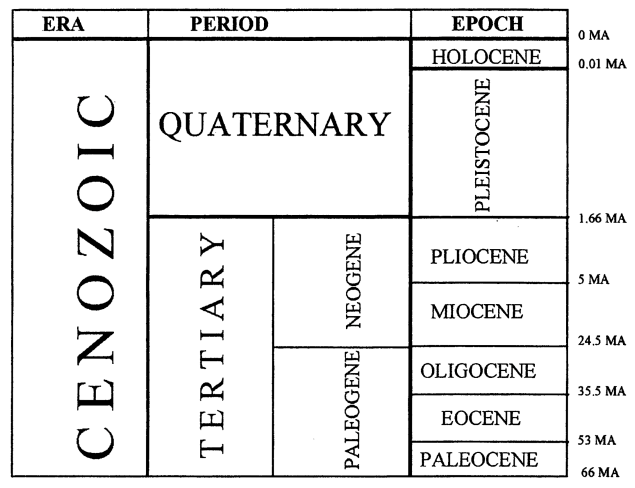

Figure 3.5: The Cenozoic time period.

These embayments were connected towards the West by a deeper seaway located in the Central Graben area. The northern embayment comprises the Norwegian-Danish Basin and the northern part of the central Graben area. The southern embayment comprises the North German Basin and the southern part of the Central Graben area. In the northern embayment, the maximum depth to the Mid-Miocene unconformity (MMU) in the Central Graben is approximately $1500 \mathrm{~m}$; these depths decrease considerably towards the east in the NorwegianDanish Basin and above the Ringkobing-Fyn High, where the Upper Cenozoic deposits are truncated by younger strata of Quaternary age. In the central part of the southern embayment, the depth to the Mid-Miocene unconformity is approximately $1000 \mathrm{~m}$, from which it decreases towards the present Dutch and German coasts and towards the southern rim of the Ringkobing-Fyn High. During the Middle Pleistocene the Ringkobing-Fyn high lost its influence on sedimentation.

The Paleocene in the North Sea Basin reflects a deep-water stage resulting from crustal subsidence combined with a general rise in the eustatic sea level. However, the southern embayment was only lit- 


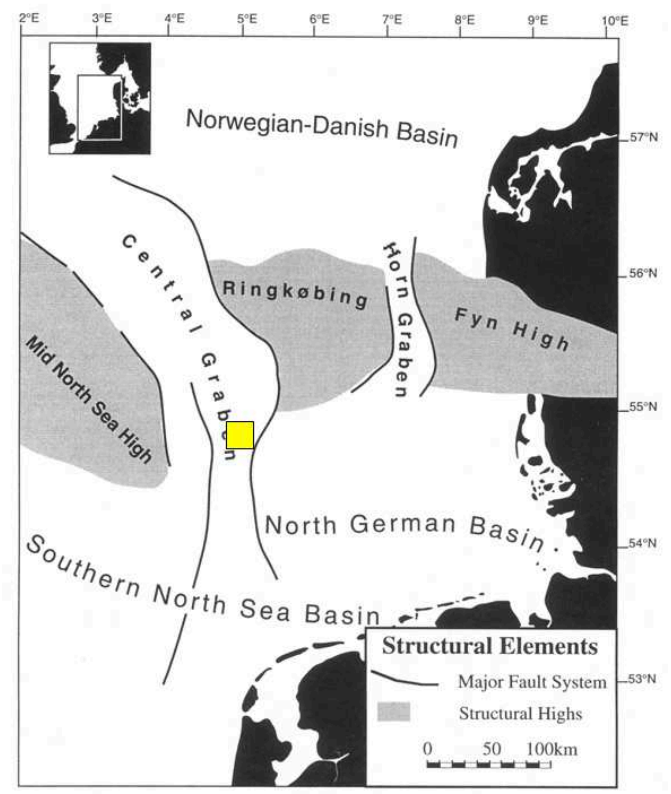

Figure 3.6: Mesozoic structures of the North Sea Basin, modified from Ziegler (1988). The location of the study area is schematically indicated by the square (F3 block coordinates: N 54 52'0.86 / E $\left.4^{\circ} 48^{\prime} 47.07\right)$.

tle influenced by regional subsidence. The transition from Paleocene to Eocene was characterized by explosive volcanic activity that was related to the northern North Atlantic-Norwegian-Greenland rifting. This extensive volcanism resulted in well-correlatable ash falls in the North Sea Basin. During the Late Eocene and Oligocene the North Sea Basin was cut off from the Tethys ocean by the uplift of the British Isles. This activity resulted in further regional uplift and relative sea level fall, resulting in a local break on sedimentation. The shallow, marine period continued until the Mid-Miocene. The Mid-Oligocene 
and Mid-Miocene sea level low-stands are reflected by truncational and onlap surfaces. The Miocene succession is generally condensed away from the coast.

During the Neogene, sedimentation rates exceeded considerably the subsidence rate and the North Sea Basin was characterized by a period of rapid deposition and shallowing of the basin (Van Boogaert and Kouwe, 1993). The most important geological event in that period was the developing of deltaic systems from the Fennoscandian Borderline prograding towards the south and southwest into the basin (Ziegler, 1990). The delta systems in the Southern North Sea region can be categorized into two groups according to their sediment source. Until the Early Pliocene, the main transport agent was the Baltic river system that eroded the Fennoscandian High, transported it into the present Baltic Sea and deposited coarse fluvial sediments (Kay, 1993). Thereafter German rivers became the main transport agents in the southern North Sea.

In the Quaternary period, the first glacial stage (Pretiglian) caused a lowstand and consequently an important sedimentary hiatus at the Plio-Pleistocene boundary. The deltaic deposition continued into the Pleistocene with shifting depocentres that were influenced by sea level fluctuations due to repeated glaciation and deglaciation cycles.

The structural development of the region was controlled mainly by inversion tectonism in the Paleogene due to compression exerted from the collision of the Apulia and the European plates (Laramide Phase). On the other hand the local structural features are mainly gravitydriven which caused syn-depositional halokinetic movements of the Zechstein evaporates and salt-induced faulting (Clausen and Korstgard, 1993). 


\subsection{Seismic Data}

The seismic data available for this study are from the Dutch block F3 and were acquired to explore for oil and gas in Upper Jurassic-Lower Cretaceous strata (Figures 3.7 and 3.8).

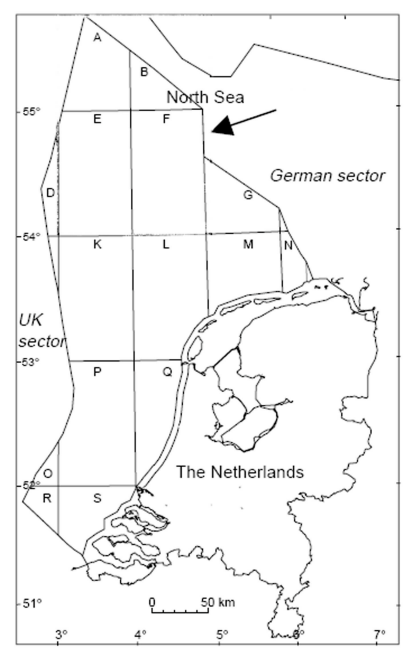

Figure 3.7: The Netherlands North Sea sector showing the location of the F3 block. Modified from Schroot et al. (2005).

The 3D seismic survey is covering an area of approximately $16 \times 24 \mathrm{~km}^{2}$. The data set of this study covers only the younger sequences and has become publicly available, accompanied by a monograph of Aminzadeh and de Groot (2006). The seismic data are post-stack timemigrated data and therefore a function of two-way travel time. A standard seismic data processing sequence was applied to the data. The data volume consists of 646 in-lines and 947 cross-lines. The inline length is $23678.35 \mathrm{~m}$ and the in-line interval is $25 \mathrm{~m}$. The cross-line length is $16124.43 \mathrm{~m}$ and the cross-line interval is $25.03 \mathrm{~m}$. The sampling rate is $4 \mathrm{~ms}$ and the number of samples per trace is 462 . The 


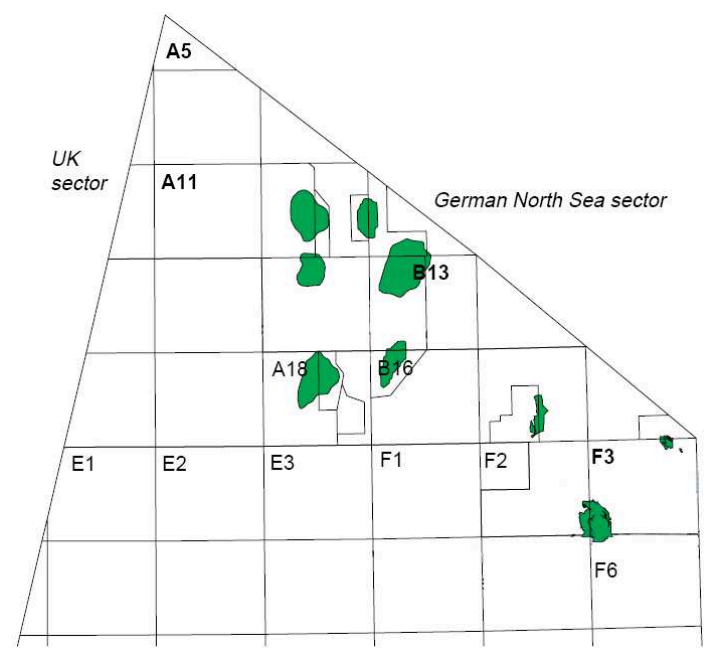

Figure 3.8: The northern part of the Dutch sector of the North Sea. Block F3 is seen to the right. Locations of oil, gas and condensate fields are indicated in gray. Modified from Schroot et al. (2005).

data was resampled with sampling rate $1 \mathrm{~ms}$. The survey basemap is shown in Figure 3.9.

\subsection{Well Data}

Well logs from four wells are available in the area. Their locations are shown on the basemap of the seismic survey (Figure 3.9) and the well names and coordinates are summarized in Table 3.1.

All wells have sonic and gamma-ray logs and only two wells (F02-01 and F03-02) have density logs measured. These logs were used to train a neural network that was then applied to the wells F03-04 and F0601 to predict densities from sonic and gamma-ray logs (Figure 3.10). Porosity $(\phi)$ was in all cases calculated from density $(\rho)$ assuming a 


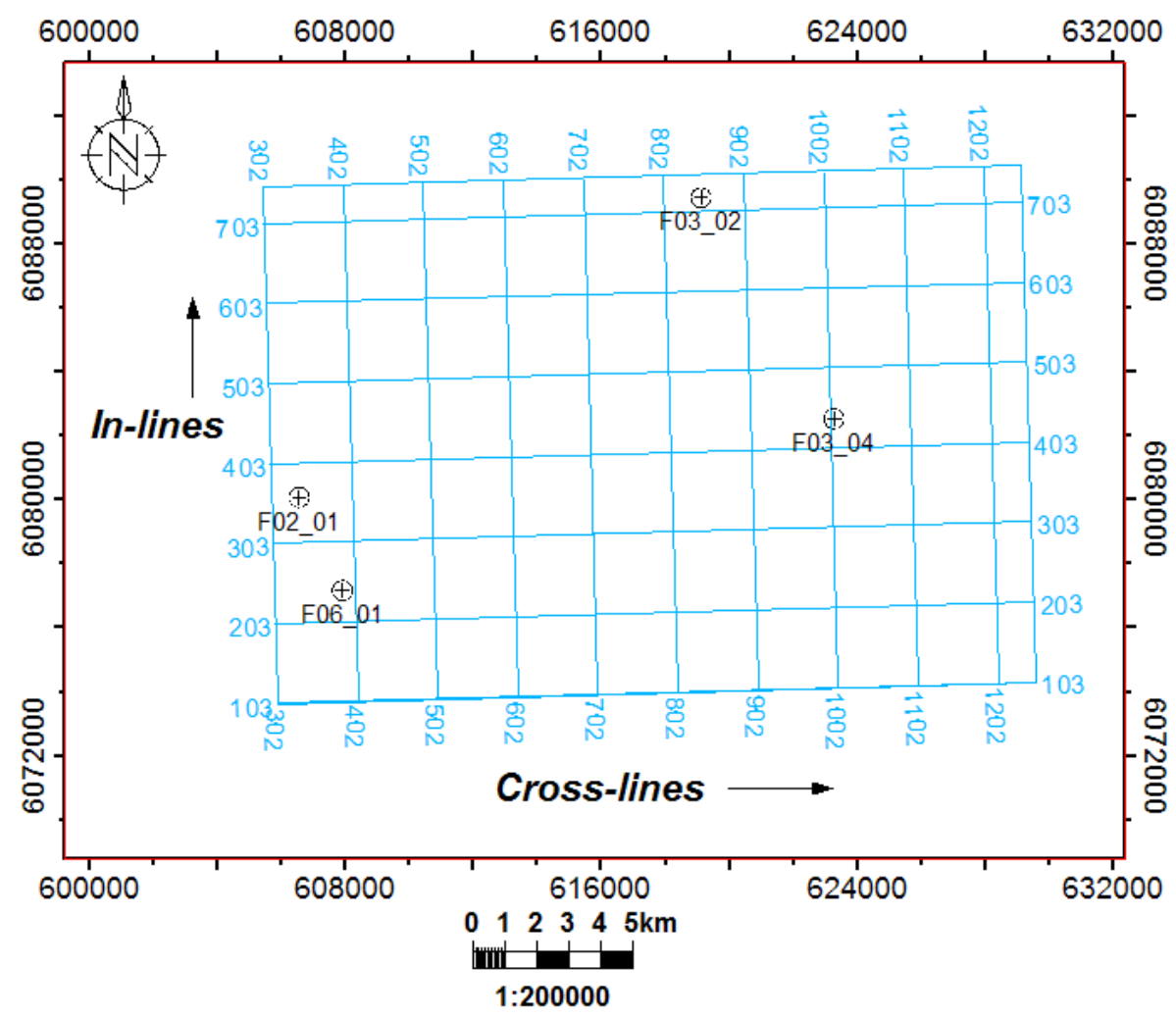

Figure 3.9: Basemap of the seismic survey.

\begin{tabular}{|c|c|c|c|c|}
\hline Well name & $\begin{array}{c}\text { Surface X } \\
\mathrm{m}\end{array}$ & $\begin{array}{c}\text { Surface Y } \\
\mathrm{m}\end{array}$ & $\begin{array}{c}\text { Start depth } \\
\mathrm{m}\end{array}$ & $\begin{array}{c}\text { End depth } \\
\mathrm{m}\end{array}$ \\
\hline \hline F03-04 & 623256.0000 & 6082586.0000 & 30.0000 & 1899 \\
\hline F06-01 & 607903.0000 & 6077213.0000 & 29.2608 & 1699 \\
\hline F03-02 & 619101.0000 & 6089491.0000 & 30.0270 & 2139 \\
\hline F02-01 & 606554.0000 & 6080126.0000 & 30.0000 & 3150 \\
\hline
\end{tabular}

Table 3.1: Location of the wells 
matrix density of $2.65 \mathrm{~g} / \mathrm{cc}$, using the relation:

$$
\phi=(2.65-\rho) /(2.65-1.05) .
$$

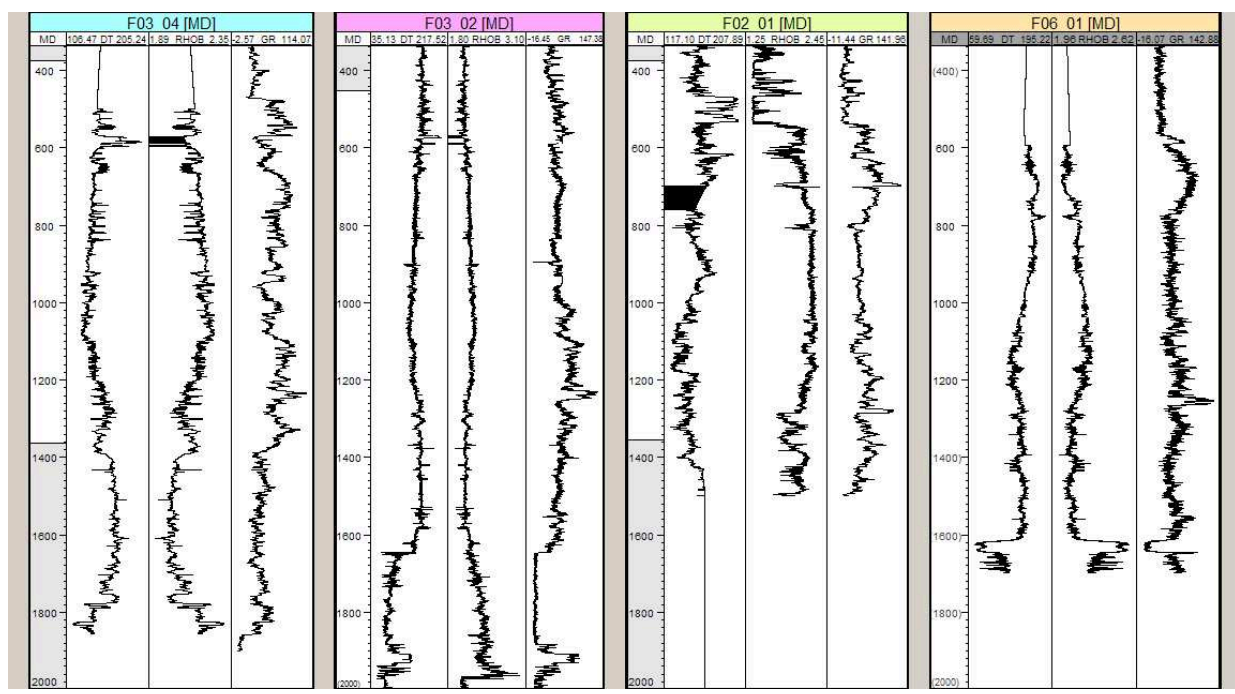

Figure 3.10: Data from the wells: F03-04, F03-02, F02-01, F06-01. From left to right for each well: Sonic, Density and Gamma ray logs.

\subsection{Data Interpretation}

The Cenozoic succession can be subdivided into two main packages, separated by the Mid-Miocene Unconformity MMU (Figure 3.11). They are distinct in their reflection characteristics, i.e., an indication for different environments of deposition ${ }^{3}$.

\footnotetext{
${ }^{3}$ This section contains excerpts from a paper on Cenozoic deposits in the North Sea by Steeghs et al. (2000).
} 

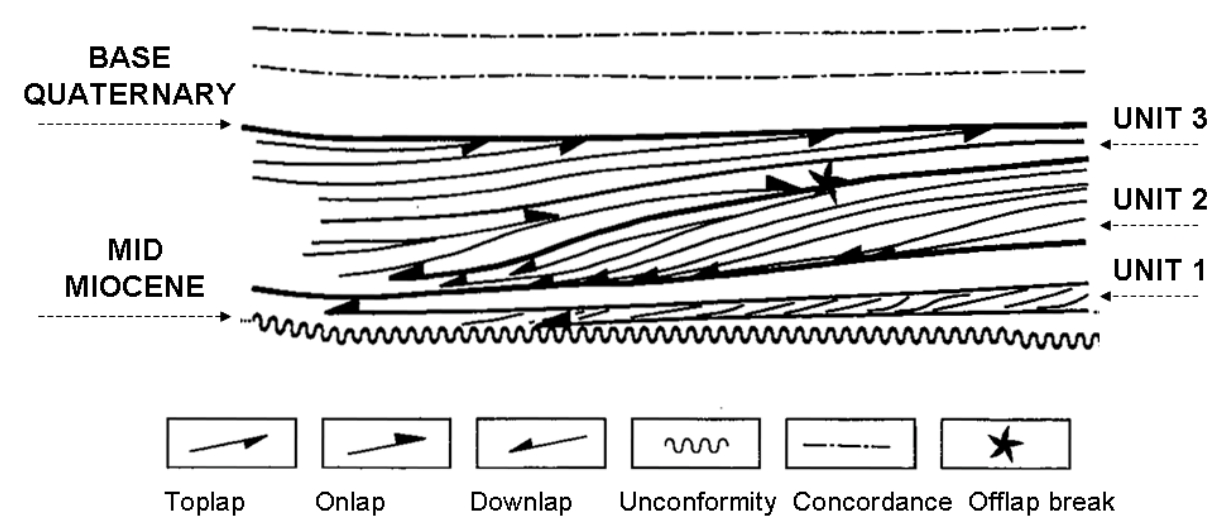

Figure 3.11: Summary of the main seismic sequence boundaries and seismic sequences in the data volume. Modified from Steeghs et al. (2000).

The seismic reflections between the base-Tertiary and the Mid-Miocene unconformities are classified as package one, which corresponds to the Lower Cenozoic succession. The reflections from the Mid-Miocene unconformity up to the sea bottom reflection form the Upper Cenozoic Succession. This was further subdivided into two main packages, as package two and package three. Package two consists of the reflections from the layers between the unconformity and the Plio-Pleistocene boundary. The Plio-Pleistocene boundary is the first parallel reflection to the unconformity after a thick zone of divergent reflections. Package three corresponds to the Quaternary succession. Package two is of most interest for us and therefore a more detailed seismic interpretation is given in the following.

Package two consist of Neogene sedimentary units of the Upper Cenozoic. The geometry of the reflection in it is much more complicated than that in the Lower Cenozoic Succession. The sequence boundaries 
of the package on the seismic sections are identified by onlaps in the up-dip direction and by downlaps in the down-dip direction. Package two is a deltaic sequence which is subdivided into three sub-sequences, subsequently termed Units 1, 2, and 3 and corresponding to three different stages of delta evolution in chronological order (Figure 3.12).

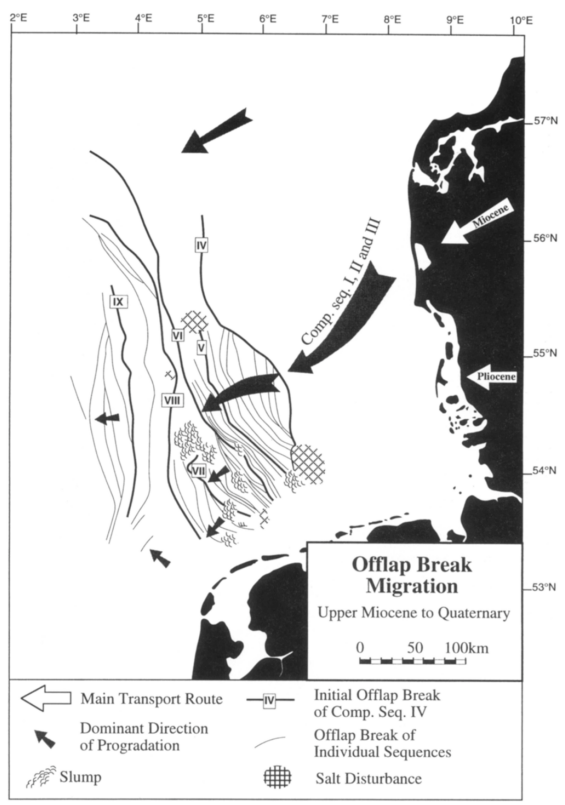

Figure 3.12: Map showing the position of the offlap break during Late Miocene to Quaternary. The offlap break migrated hundreds of kilometers into the southern embayment. From Sørensen et al. (1997).

Unit 1: This unit belongs to the first phase of the delta evolution above the unconformity. The lower part of it is considered to consist of an Upper Middle Miocene strata which onlaps onto the Mid-Miocene unconformity in the eastern part and downlaps to the west. The source of sediment supply is in the eastern part of the area. In the direc- 
tion of downlap there is an increasing nondepositional hiatus above the unconformity surface. This is determined by the successive terminations of downlapping strata towards the west. The Upper Middle Miocene strata fill a subtle depression caused by a salt diapir. Above the Late Middle Miocene onlapping and downlapping units, a prograding reflection configuration shows significant deposition due to lateral outbuilding. The middle segment of the Unit 1 has clinoforms, with a shingled reflection pattern. A shingled reflection configuration is a thin prograding seismic pattern, commonly with parallel upper and lower boundaries (topsets and bottom sets) and with gently dipping parallel internal reflectors, i.e., clinoforms that terminate by apparent toplap and downlap. The successive clinoforms within unit 1 show little overlap with each other and are evidence of progradation into shallow water. The angle and the height of the clinoforms provide an idea on the water depth during deposition. Measurements show that their height varies between 4 and $10 \mathrm{~ms}$ two-way travel time $(\approx 3-9 \mathrm{~m})$, which indicates a very shallow water depth during deposition. The reflection strength values do not vary considerably along the clinoforms, which is an indication for relatively homogeneous sedimentary material. The high values of the reflection strength can indicate highstand deposits (Gregersen, 1997).

Unit 2: This sequence belongs to the second phase of delta evolution. The direction of progradation is towards the southwest during the deposition (Sequence V in Figure 3.12) . The inclination of the reflection pattern is steeper than in other delta units and decreases towards the North. It consists of sigmoid progradational reflection configurations. This unit shows a prograding clinoform pattern formed by superimposed sigmoid reflections. Stratigraphically it is interpreted as thin, gently dipping strata with upper and lower segments-topsets and bottomsets, and more steeply dipping thicker middle segmentsclinoforms. The topsets have a concordant relation with the overlying 
surface. The clinoforms form a lens-shaped depositional unit prograding in the downdip direction. The bottomsets approach to the underlying surface at very low angles where the strata become very thin. The concordant relation of the topsets with the overlying unit shows that the aggradation of the topsets was coincident with the progradation of the clinoform. This configuration is evidence of the relatively low sediment supply and rapid basin subsidence to allow deposition and preservation of the topset units. It is also attributed to the relatively low energy sedimentation regime. The reflection strength values of Unit 2 increase upwards.

Unit 3: This unit belongs to the end phase of delta evolution. The reflection configurations of the unit are characterized by divergence. The areal extent of the unit has a wedge shape. The divergent characteristic of the unit is attributed to the lateral variation in the rate of the deposition. In the West and Southwest the strata are thinning and locally getting below the resolution of the seismic. The lower sequence boundary of the unit onlaps onto the upper sequence boundary of Unit 2 in the East, which is an indication for a relative sea level rise. This toplap is evidence of a nondepositional hiatus, sedimentary bypassing, or erosion. It is related to the sea level fall during the first glacial period at the Plio-Pleistocene boundary. The truncation of the reflections at this boundary is associated with the occurrence of channels.

Hummocky clinoforms are observed in the bottom segment of Unit 3, at the left side of the lower segment of the sigmoidal clinoforms from Unit 2. This configuration consists of subparallel reflection segments with non-systematic reflection terminations. This package represents a syn-sedimentary deformation that was caused by the reactivation of the Permian salt dome. The hummocky clinoforms also indicate a transitional phase from high-energy basin conditions to a much lower 
energy regime. The interpretation of the well data was taken from the monograph of Aminzadeh and de Groot (2006). The principal horizons of the Upper Cenozoic package were correlated between the wells. For the sake of convenience the names of the seismic horizons are the same as in the monograph.

\subsubsection{Clinoform Mapping}

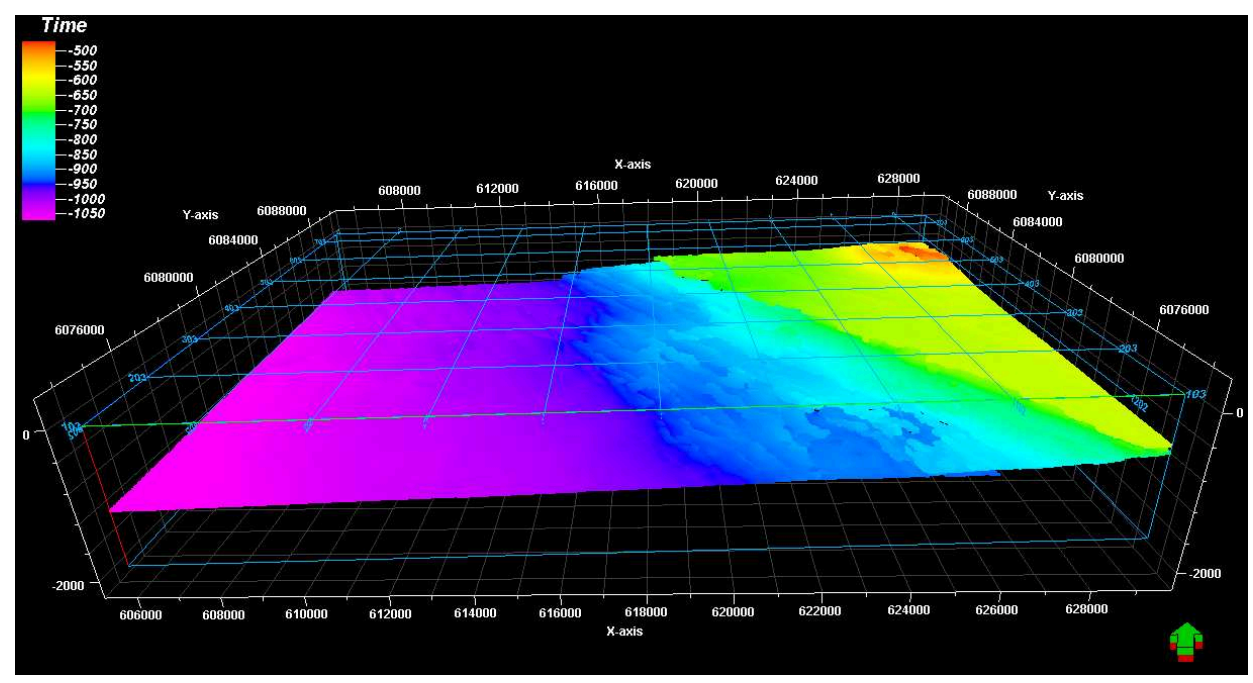

Figure 3.13: The two-way travel time values of the mapped clinoform surfaces.

The clinoforms of the Lower Pliocene (Unit 2) were first analyzed by visual inspection. For a systematic sequence stratigraphic interpretation several horizons were selected in the clinoforms to map the progradational direction and the patterns of the clinoforms. The mapped clinoform surfaces are displayed in Figure 3.13.

The two-way travel time values along the clinoforms are increasing 
towards North and Northwest and the clinoforms are prograding from East-Northeast towards West-Southwest. These results coincide well with the geological history of the region (see Figure 3.13).

\subsection{Seismic Data Selection for Inversion}

The selection of a seismic line from the total data volume is performed on the basis of several criteria:

- Unit 2 should be present.

- The seismic line should cross Unit 2 along the progradational direction, so that the sigmoidal shapes of deltaic sediments have an optimum expression.

- Internal structures of Unit 2 should clearly be visible, so that high-resolution inversion technique can be tested.

- The line should be close to at least one well so that a priori information for the inversion is available.

\subsubsection{Reference Reflections}

Reference reflections are required to clearly indicate the position of the inversion interval on the seismic data. A look at the seismic in-line section, which coincides with the progradational direction of the clinoform sequence, reveals the presence of two strong seismic reflections confining the target zone, i.e., Unit 2.

First, the older horizon 'MFS4' is highlighted on Figure 3.15 in white and forms the base of the clinoform package. The second, younger reflector 'Truncation 1' is the top of the clinoform package. 'Truncation 
1' together with 'MFS4', forms the bottom and the top of the target zone (Figure 3.15).

\subsubsection{Proximity of Wells}

The selected and interpreted 3D horizon of the prograding clinoforms of the Lower Pliocene and the locations of four available wells are displayed in Figure 3.14(a). A seismic line connecting the wells F0304, F03-02, F02-01, and F06-01, with the main horizons correlation applied is shown in Figure 3.14(b).

The wells F02-01 and F06-01 are located in the southwestern part of the F3 block, at the bottom set of the clinoform sequence. The well F03-02 is situated to the North and at the topset of the sequence. The well F03-04 is located in the Eastern part of the block, at the clinoform part of the sequence.

Based on the analysis of the well locations and the clinoform propagation direction the seismic section (in-line 441) displayed in Figure 3.15 was chosen for further analysis. Preference was given to this in-line section because it coincides quite well with the propagation direction of the clinoform sequence. The well F03-04 is located close to this line, and is the only well that penetrates the target Unit 2 completely. A deficiency of this well is that the density log was not acquired in situ but recomputed from the sonic log. Most of the seismic section is in the clinoform part of the sequence and therefore contains internal structures. Additionally, the section is of good quality.

The sonic, gamma ray and recomputed density logs of the Well F03-04 are rather spiky and required some smoothing. A box-shaped averaging filter was therefore applied with a length of $2 \mathrm{~m}$ and in which the central sample was replaced by the average of all samples within this window. The smoothening filter was applied to the entire length to re- 


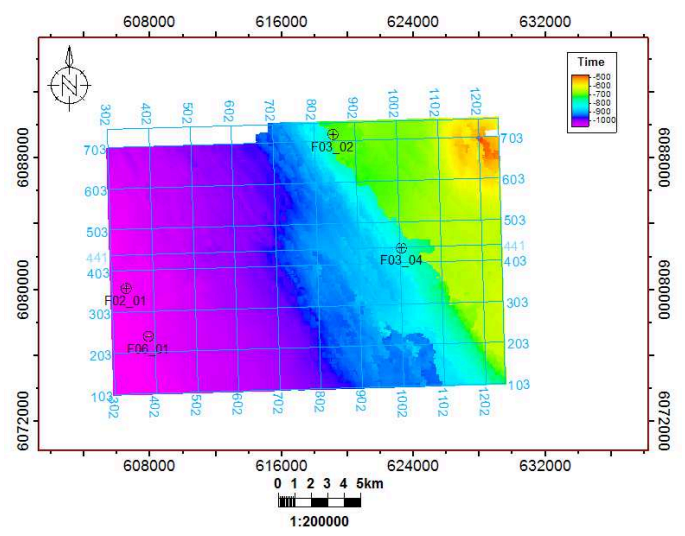

(a)

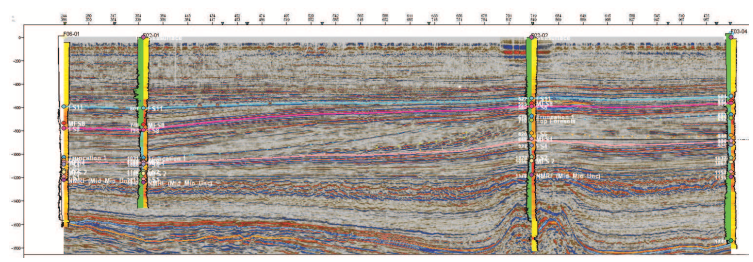

(b)

Figure 3.14: (a) The interpreted 3D horizon of the prograding clinoforms and the locations of four available wells (F06-01, F02-01, F03-02 and F03-04), together with the location of the seismic section selected for inversion (in-line 441); (b) a seismic line connecting these four wells, with the main horizons shown in colors (Aminzadeh and de Groot, 2006). 'Truncation 1' (blue dashed) and 'MFS4' (pink) indicate the top and bottom of the clinoform package (the target zone). The vertical axis is the two-way travel time in ms; the horizontal axis shows the in-line and cross-line numbers. Blue are negative and brown are positive polarities. 


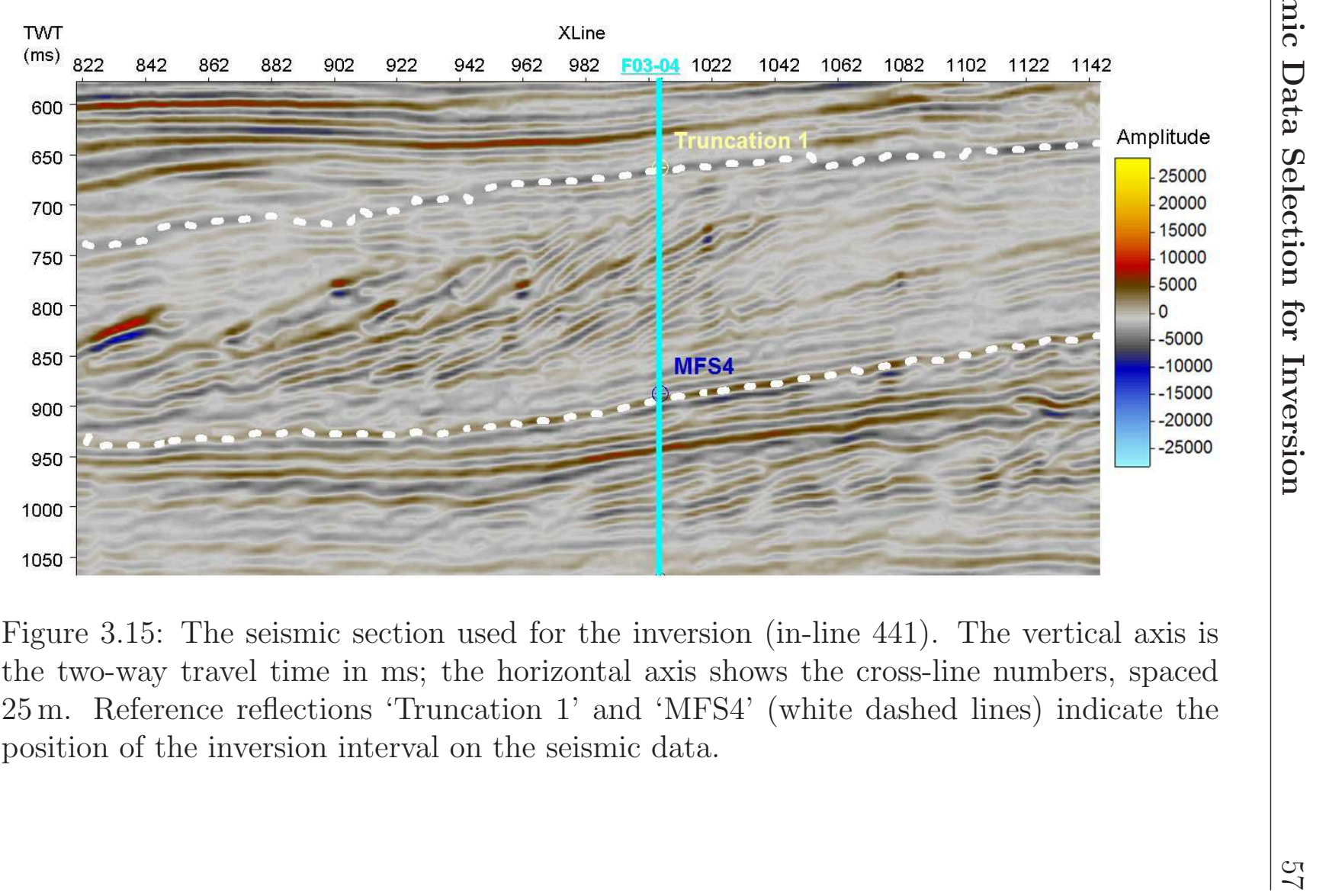




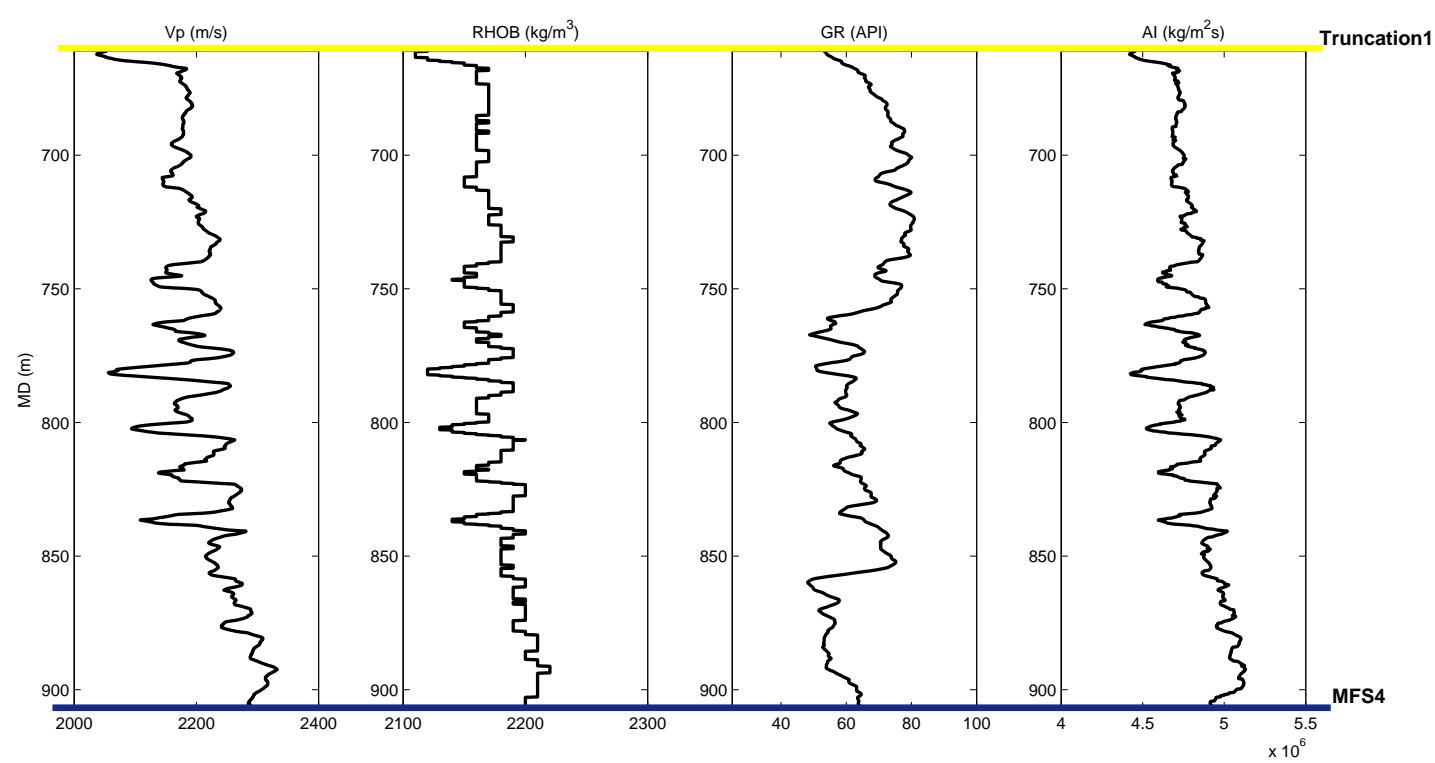

Figure 3.16: The P-wave velocity and density (recalculated from sonic logs), gamma-ray and acoustic impedance logs of the well F03-04. 'Truncation 1' and 'MFS4' indicate the top and the bottom of the clinoform package (target zone). 
duce noise and remove spikes. The smoothed logs are shown in Figure 3.16 .

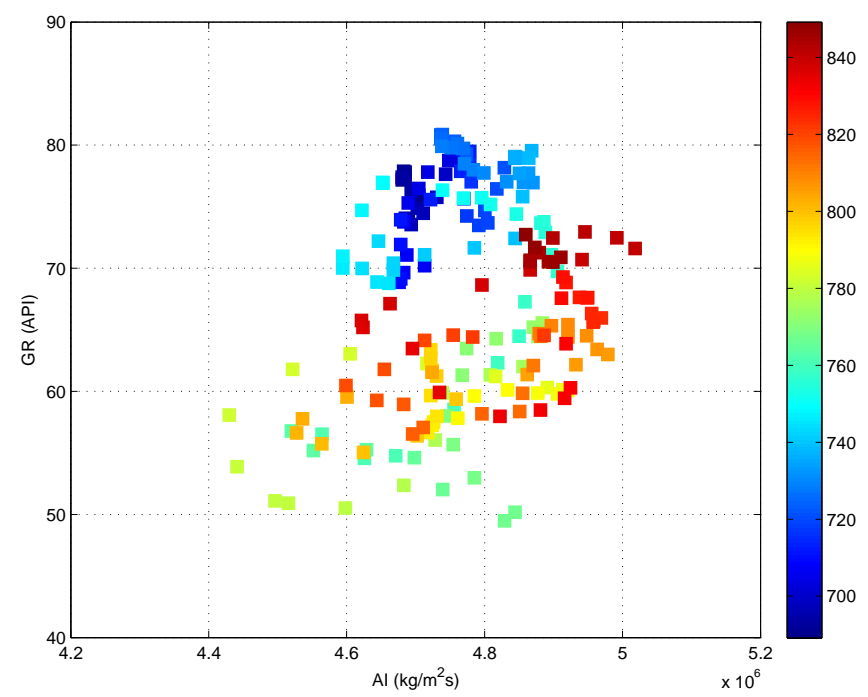

Figure 3.17: A crossplot of the gamma-ray values against acoustic impedances values within the target zone; the color scale is assigned to the points as a function of depth (in meters). For discussion see text.

Figure 3.17 shows a crossplot of the gamma-ray values against acoustic impedance values (computed from the velocity and density logs) within the target zone; the color scale is assigned to the points as a function of depth to demonstrate the correlation between the two logs. There are two types of sediments that can be clearly distinguished from the plot: shale-rich sediments with generally higher gamma-ray values (bluish cloud, correlation coefficient 0.53) that belong to the upper part of the target zone $(694-750 \mathrm{~m})$ and sand-rich sediments with generally low gamma-ray values (reddish cloud, correlation coefficient 0.56 ) that belong to the lower part of the target zone $(750-850 \mathrm{~m})$. In addition, 
the compaction effect of the sediments can be observed, because the deeper sediments on the plot are generally characterized by higher acoustic impedance values.

\subsection{Wavelet Extraction}

There are several methods to extract wavelets from seismic data when the source wavelet is not available. The statistical extraction method used in estimating the wavelet assumes that the autocorrelation of the wavelet is the same as the truncated autocorrelation of the seismic trace. There are many factors that affect the quality of a wavelet, but mainly the quality of the seismic and the used logs. The average autocorrelation from several seismic traces is used to provide a more representative estimate. As a result, the following steps are performed:

1. Compute the Fourier transform of the autocorrelation of all selected seismic traces,

2. Average the Fourier Spectra,

3. Compute the inverse of the averaged spectra as zero-phase estimate of the wavelet.

A seismic trace can be represented by the convolutional model (equation (2.4)), which can be written as:

$$
s(t)=w(t) * r(t)+n(t),
$$

where $s(t)$ is an observed seismic trace, $w(t)$ is a wavelet to be extracted, and $n(t)$ is the error of the noise. The assumption here is that the noise does not correlate with the reflectivity and therefore is an additive component. 
The equation can be rewritten in terms of power;

$$
\left|S^{2}(f)\right|=\left|W^{2}(f)\right|\left|R^{2}(f)\right|+\left|N^{2}(f)\right| .
$$

For a single trace, all elements that compose the seismic trace are present in the wavelet, the reflectivity series and the noise. By averaging several traces together over a sufficiently large (statistical) sum, the following conditions may occur: $\left|R^{2}(f)\right|$ tends roughly towards a white noise spectrum caused by the random occurrence of $r(t)$ distributed in space. The closer $r(t)$ is to a random (i.e., 'white') series, the more $\left|R^{2}(f)\right|$ becomes constant and affects less the multiplicative operations on the average spectrum. $\left|N^{2}(f)\right|$ tends to some average noise spectrum. Its effect will mainly depend on the signal/noise ratio of the input $s(t)$. In good, strong signal areas, noise will be a negligible factor and have a minor additive component, which can be ignored. Given these conditions, $\left|S^{2}(f)\right|$ is a good representation of the average power spectrum of the seismic wavelet $\left|W^{2}(f)\right|$. The wavelet $w(t)$ can be computed in the time domain using the inverse Fourier transform of the square root of $\left|W^{2}(f)\right|$ under the assumption that the wavelet is zero phase. It has been tested that this assumption is valid for the post-stack time-migrated seismic data set from the North Sea, Dutch block F3.

The adoption of the convolutional model implies the assumption that the wavelet is constant in time, which is only reasonable for a small target zone. Therefore, the seismic data cube was cropped to a subvolume of 100 in-lines by 100 cross-lines around the well in the lateral directions and from 670 to $900 \mathrm{~ms}$ (the target zone Unit 2) in the vertical direction. The sampling rate of the points describing the wavelet is $1 \mathrm{~ms}$. The overall operator length of the wavelet in time is $70 \mathrm{~ms}$. The extracted wavelet is a zero-phase, symmetrical wavelet with a central frequency of $55 \mathrm{~Hz}$ and is shown in Figure 3.18(a) together 


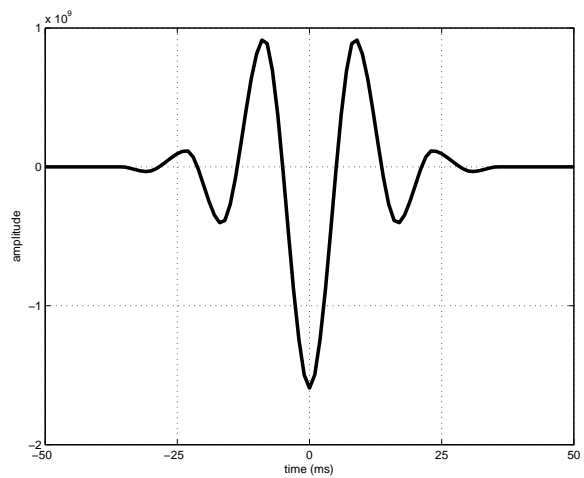

(a)

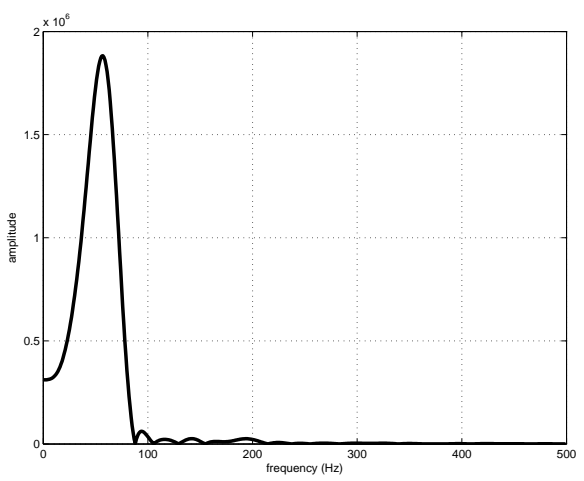

(b)

Figure 3.18: (a) The extracted wavelet and (b) its spectrum.

with its spectrum in Figure 3.18(b). 


\section{Chapter 4}

\section{Stratigraphic model-based, low-parametrization seismic inversion}

\subsection{Introduction}

For this method, three major steps are performed (Figure 4.1). First, the thickness and acoustic impedance of the layers that compose the clinoform package are estimated from well logs with a resolution in the order of tens of $\mathrm{cm}$. Second, the entire clinoform package is geometrically modeled based on the seismic data in the area of interest. Third, the clinoform models will guide how the a priori knowledge derived from the well logs propagates from trace to trace away from the well. The geometric clinoform model is also used to map the solution of the last processed trace to the initial guess of the next trace. This way the complete clinoform sequence is characterized at a sub-seismic scale. 
Stratigraphic model-based, low-parametrization seismic inversion

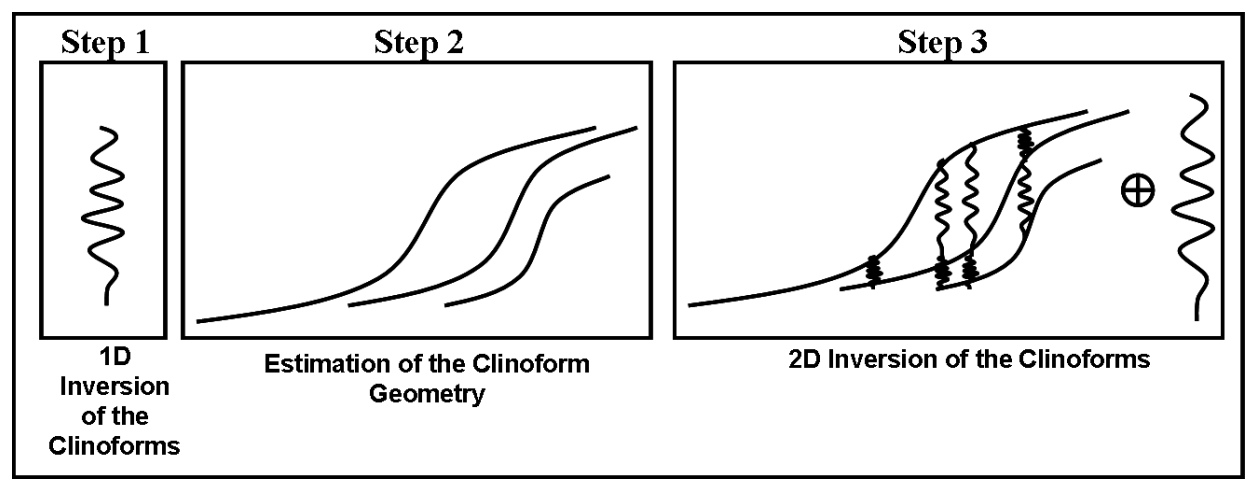

Figure 4.1: The workflow of the stratigraphy-based, lowparameterization seismic inversion method.

\subsection{D Inversion}

Getting a structural image of the main layer structure from which the properties of the clinoform sequence can be retrieved is the primary focus in this step of single trace inversion.

\subsubsection{Formulation of the inverse problem}

The inversion method described in Chapter 2 is used as a basis for single trace inversion. We use a one-dimensional convolution between a layered model and the wavelet as forward model (equation (2.4)). The wavelet extracted from the measured data set (Chapter 3) serves as the source wavelet. The subsurface is assumed to consist of a stack of locally planar, homogeneous layers. The time thickness of the layers $T_{j}$, is derived from the two way travel time $\tau_{j}$, as follows

$$
T_{j}=0.5\left(\tau_{j}-\tau_{j-1}\right) .
$$

The parameter vector $\boldsymbol{x}$ consists of the acoustic impedances $Z_{j}$ and 
the corresponding time thicknesses of the layers $T_{j}$.

$$
\boldsymbol{x}=\left[Z_{1}, \ldots, Z_{N}, T_{1}, \ldots, T_{N}\right],
$$

where $N$ is the number of layers.

\subsubsection{A priori Information}

\section{A prior mean}

We use the sonic and density logs (recalculated from the sonic log) of the well F03-04 as source of a priori information. The sonic log and check shots are used to convert the well data from depth to time in order to relate the well data to the seismic. In order to extract as much a priori information as possible from the well data an impedance log computed from the velocity and density logs serves as the a priori mean vector $\boldsymbol{x}_{i}$ (see equation (2.8)).

$$
\boldsymbol{x}_{i}=\left[Z_{1}^{i}, \ldots, Z_{n}^{i}\right]
$$

where $n=1200$ is the number of samples in the log.

\section{A priori uncertainties}

The expected uncertainties in the parameters to be estimated can be represented by the a priori covariance matrix. In case when only a single well is available in the area of investigation, we use a histogram of a well log obtained from the target zone only. We use the standard deviation of the best-fit Gaussian distribution to the histogram of the acoustic impedances as an estimate of the uncertainty. Based on equation (2.11), the a priori covariance matrix is:

$$
\boldsymbol{C}_{\boldsymbol{x}}=\sigma_{\boldsymbol{x}_{\boldsymbol{i}}}^{2} \mathbf{I}
$$




\section{Stratigraphic model-based, low-parametrization seismic}

with $\mathbf{I}$ the identity matrix. The noise covariance matrix is estimated from the seismic trace $\boldsymbol{y}$,

$$
\boldsymbol{C}_{\boldsymbol{n}}=\sigma_{\boldsymbol{y}}^{2} \mathbf{I}
$$

\subsubsection{Initial Model}

As explained in Chapter 2, the optimization scheme starts with an initial guess $\boldsymbol{x}_{0}$ of the model parameters, from which an initial model response can be computed. The initial model $\boldsymbol{x}_{\mathbf{0}}$ is a layer model at the location of the well and is therefore called 'Well Model'.

$$
\boldsymbol{x}_{0}=\left[Z_{1}^{0}, \ldots, Z_{N}^{0}, T_{1}^{0}, \ldots, T_{N}^{0}\right] .
$$

This model is a 'blocked log' in which each interval with constant properties represents a layer with a particular lithofacies.

\section{Lithofacies}

The log interpretation is conducted by traditional comparison of the gamma-ray and compressional velocity logs, because an in-situ measured density log was absent. A basic rule for gamma-ray log interpretation is that the lower log values correspond to more sand-rich layers and higher values correspond to more shale-rich layers (Luthi, 2001). The layer identification was complicated because shale and sand had comparable acoustic properties. In addition, the vertical resolution of the seismic data is in the order of tens of meters, whereas the resolution of the well data is in the order of tens of centimeters. As a result, the 'Well Model' was derived consisting of $N=27$ individual layers with typical thicknesses of 3-14 $\mathrm{m}$ and with layer-lithologies alternating between different proportions of shale and sand. The acoustic impedances of the 'Well Model' were then averaged (arithmetic mean) within each layer. Figure 4.2 shows the log data and a schematic 


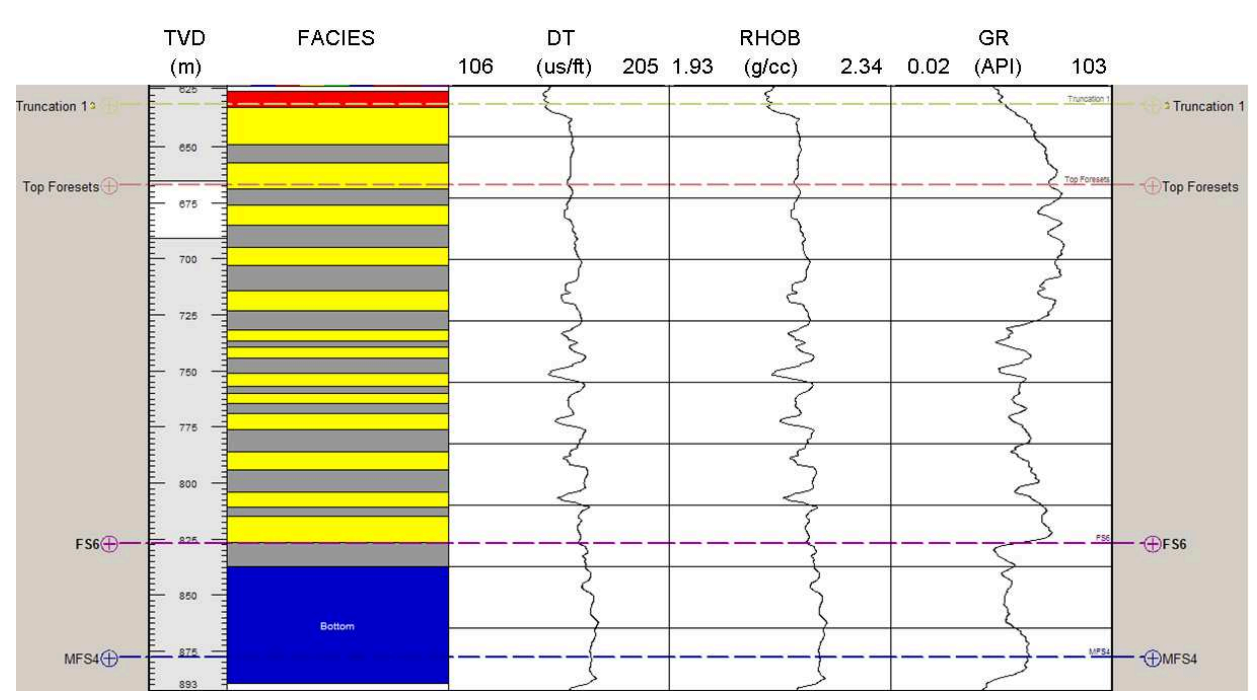

Figure 4.2: The 'Well Model', with yellow denoting sand-rich and gray denoting shale-rich layers. 'Truncation 1' (yellow) and 'MFS4' (blue) indicate the top and the bottom of the clinoform package (target zone). The curves on the right are the compressional velocity, bulk density and gamma ray logs.

representation of the 'Well Model'. In the seismic traces, only layers situated within half the wavelength upwards and downwards from the target zone have an impact on the measured seismic reflection in the target zone. Therefore the transitions just below and just above the target zone were included in the 'Well Model'. The 'Well Model' properties are summarized in Table 4.1. The initial 'Well Model' superimposed on the impedance log is shown in Figure 4.3. It shows not only the layering but also the increase in acoustic impedance with depth (time) due to compaction. 
Stratigraphic model-based, low-parametrization seismic

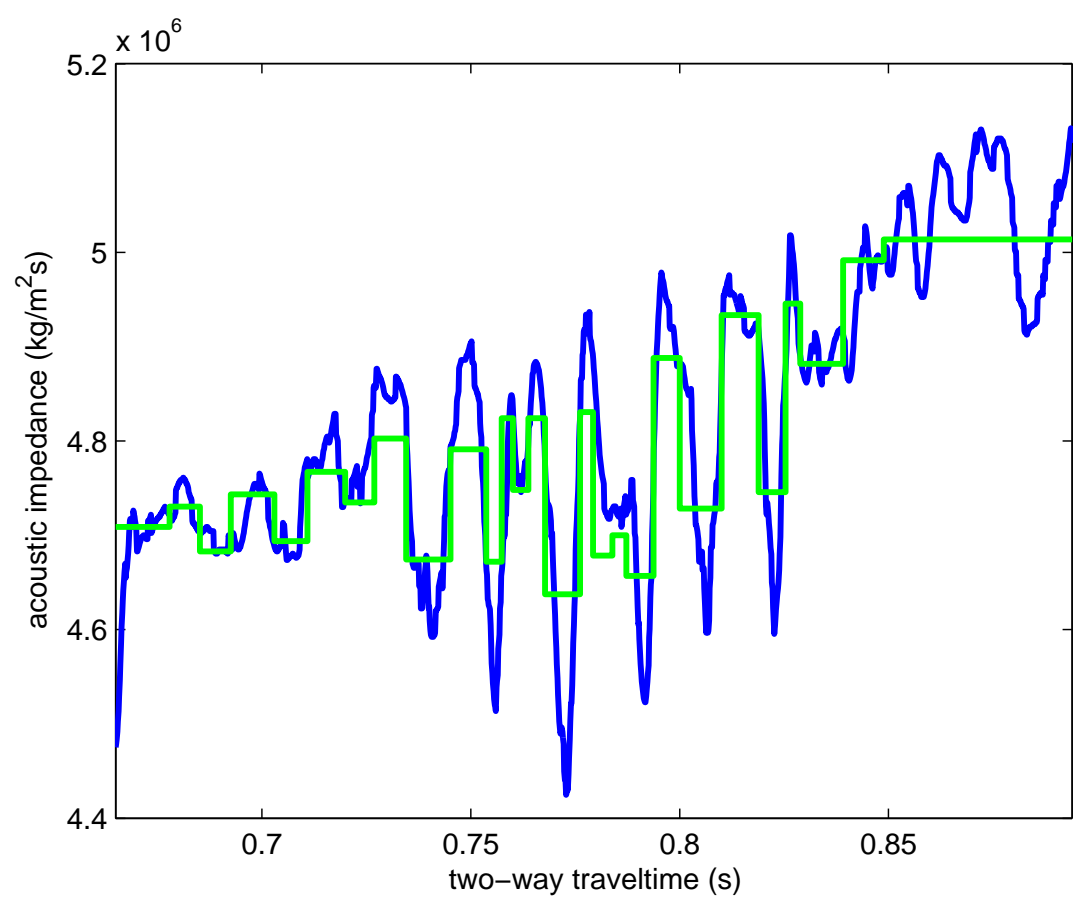

Figure 4.3: The initial 'Well Model' (based on well data) superimposed on to the impedance log (blue).

\section{Seismic validation}

The initial 'Well Model' was based on the interpretation of well logs and is independent of the seismic data. To validate the model, the lithofacies log was superimposed on in-line 441 of the seismic data (Figure 4.4(a)) as well as to the closest cross-section 1006 (Figure 4.4(b)). Figure 4.4 shows a good correlation between the measured seismic data and the lithofacies log; thereby supporting the choice of the 'Well Model'. 


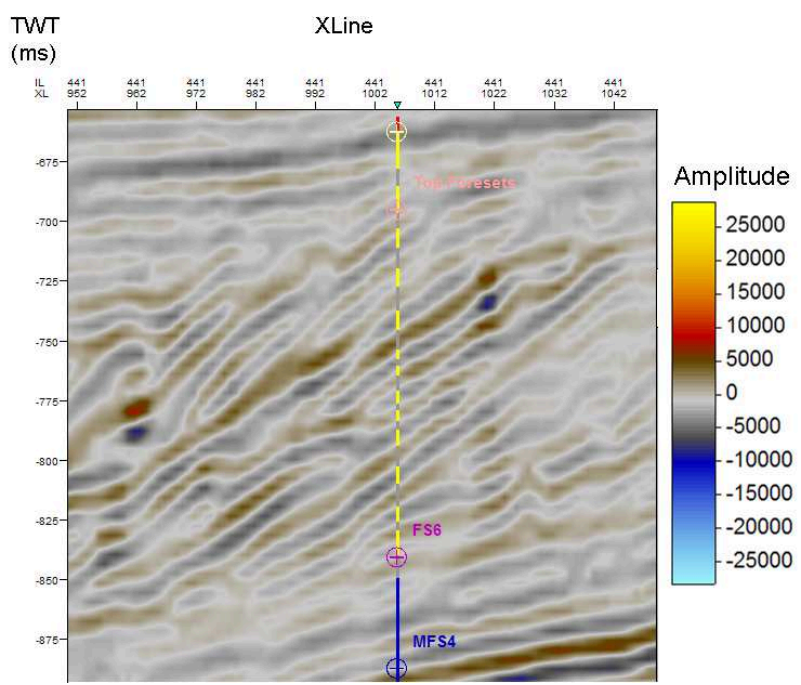

(a) inline section 441

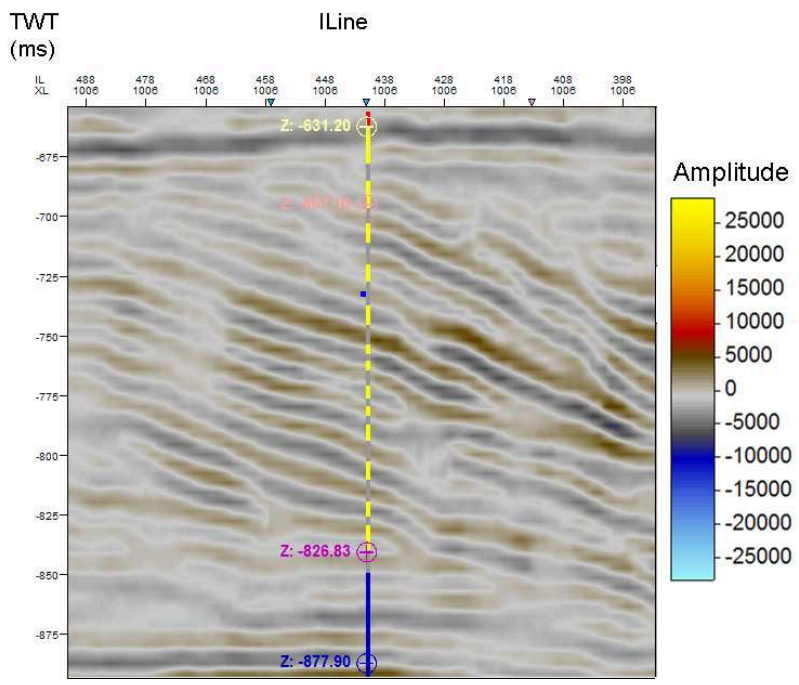

(b) cross-section 1006

Figure 4.4: The 'Well Model' lithofacies log (with yellow denoting sand-rich and gray shale-rich layers) superimposed (a) on in-line section 441 of the seismic data as well as (b) on the closest cross-line 1006(b) to validate the choice of the model. Scales and axes as in similar previous figures. 
Stratigraphic model-based, low-parametrization seismic 70 inversion

\begin{tabular}{|c|c|c|c|c|}
\hline $\begin{array}{c}\text { TVD } \\
\mathrm{m}\end{array}$ & $\begin{array}{c}\text { Thickness } \\
\mathrm{m}\end{array}$ & $\begin{array}{c}\text { Thickness } \\
\mathrm{ms}\end{array}$ & $\begin{array}{c}\mathrm{AI} \\
\left(\mathrm{kg} / \mathrm{m}^{2} \mathrm{~s}\right)\end{array}$ & $\begin{array}{c}\text { Reference } \\
\text { Horizon }\end{array}$ \\
\hline \hline 626 & 9 & 5 & 4419941 & \\
\hline 635 & 14 & 6 & 4708836 & Truncation 1 \\
\hline 649 & 8 & 4 & 4730456 & \\
\hline 657 & 8 & 4 & 4683224 & \\
\hline 665 & 11 & 5 & 4743428 & Top foresets \\
\hline 676 & 9 & 4 & 4693192 & \\
\hline 685 & 10 & 5 & 4767017 & \\
\hline 695 & 8 & 3 & 4733769 & \\
\hline 703 & 11 & 5 & 4801555 & \\
\hline 714 & 9 & 4 & 4675278 & \\
\hline 723 & 9 & 4 & 4791077 & \\
\hline 732 & 4 & 2 & 4672978 & \\
\hline 736 & 3 & 1 & 4825122 & \\
\hline 739 & 4 & 2 & 4749050 & \\
\hline 743 & 8 & 3 & 4824060 & \\
\hline 751 & 6 & 3 & 4638937 & \\
\hline 757 & 5 & 2 & 4830608 & \\
\hline 762 & 3 & 1 & 4721810 & \\
\hline 765 & 4 & 2 & 4765329 & \\
\hline 769 & 7 & 3 & 4656473 & \\
\hline 776 & 10 & 5 & 4887751 & \\
\hline 786 & 8 & 4 & 4728999 & \\
\hline 794 & 10 & 4 & 4934651 & \\
\hline 804 & 7 & 3 & 4746176 & \\
\hline 811 & 4 & 2 & 4946307 & \\
\hline 815 & 11 & 5 & 4882398 & \\
\hline 826 & 11 & 5 & 4992362 & FS6 \\
\hline 837 & 52 & 23 & 5013720 & \\
\hline 889 & 13 & 6 & 4719054 & MFS4 \\
\hline & & & & \\
\hline
\end{tabular}

Table 4.1: The properties of 'Well Model'. 


\subsubsection{Optimization}

The position of internal boundaries of the clinoform sequence can be quite ambiguous. Therefore, after the 'Well Model' is established, the model parameters are iteratively refined by minimizing equation (2.17). Due to the absence of gradient information we used a simplex optimization method as described in Section 2.4.1.

The absolute scale of the wavelet is often unknown due to the fact that the source wavelet is estimated from the seismic signal and that arbitrary scaling factors may have been used in data processing. Therefore the resulting amplitude of the wavelet extracted from the seismic data (Chapter 3) was scaled in such a way that it minimizes the difference between the processed seismic data $\boldsymbol{y}$ and synthetic data $\boldsymbol{g}(\boldsymbol{x})$.

\subsubsection{Regularization}

As was mentioned in Chapter 2, for a linear inverse problem the optimal value for the regularization parameter $\lambda$ can be found by constructing and analyzing a so-called 'L-curve'. Although we are handling a non-linear inverse problem, a similar approach was applied. Taking into account the fact that the data mismatch term and a priori mismatch term are of the same order of magnitude, we expect that the optimal $\lambda$ value will lie around 1 . This led to a choice of $\lambda$ values for the 'L-curve' test as described below:

- $\lambda$ from 0.0 to 0.1 with a step size of 0.01 ;

- $\lambda$ from 0.1 to 2 with a step size of 0.1 ;

- $\lambda$ from 2 to 10 with a step size of 1 ;

- $\lambda$ from 10 to 100 with a step size of 10 ; 
Stratigraphic model-based, low-parametrization seismic inversion

Very small and very large values of $\lambda$ were chosen to illustrate the effects when the data are fitted well but the reconstructed image is not smooth and vice versa. For the given range of $\lambda$ values $\log \|\boldsymbol{y}-\boldsymbol{g}(\boldsymbol{x})\|_{2}^{2}$ and $\log \left\|\boldsymbol{x}-\boldsymbol{x}_{i}\right\|_{2}^{2}$ were computed. The results are plotted on a double logarithmic scale, $\lambda$ values are increasing from the top-left to the bottom-right in Figure 4.5. As can be seen, the bend in the curve is not sharp, and therefore it is not easy to determine an optimal solution. Instead, a group of solutions inside the circle of Figure 4.5 was selected. These solutions lie slightly to the right of the position of largest upwards-pointing curvature. This set of solutions corresponds to $\lambda$ values ranging from 0.3 to 0.9 .

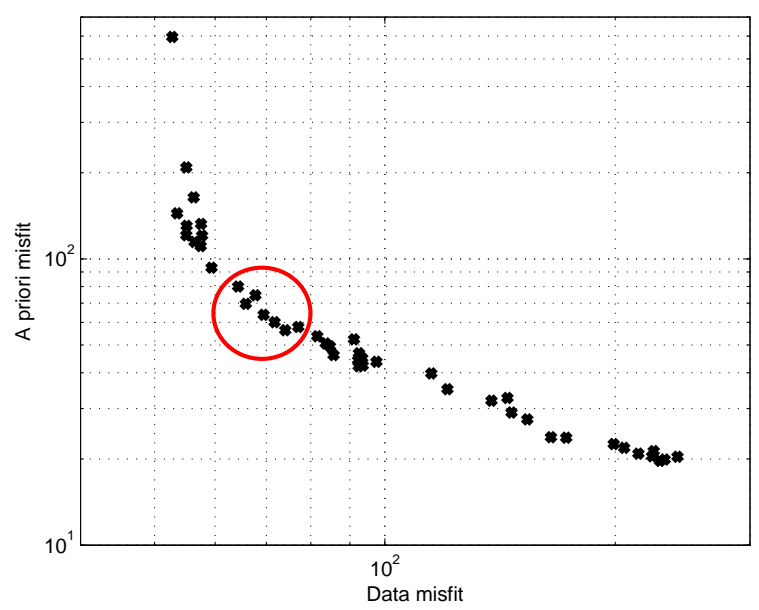

Figure 4.5: $\log \left\|\boldsymbol{x}-\boldsymbol{x}_{i}\right\|_{2}^{2}$ versus $\log \|\boldsymbol{y}-\boldsymbol{g}(\boldsymbol{x})\|_{2}^{2}$ for $\lambda$ values in the range from 0.001 to 100 (increasing from the top-left to the bottomright). A group of possible optimal solutions are indicated inside the circle.

We extended the range for the 1D inversion results of the seismic trace closest to the well to values in the range from 0.1 to 1.1 . The results 
for all tests are shown in Figure 4.6 in two columns. The left column of Figure 4.6 illustrates the difference between the 'Well Model' (green) and the estimated model (red) superimposed on the actual impedance log from the well (blue). The right column of Figure 4.6 shows the synthetic seismic based on the 'Well Model' (green) and the synthetic seismic (red), respectively, superimposed on the measured seismic data (blue).

\subsubsection{Results}

The estimated impedances and layer thicknesses for different values of $\lambda$ within the chosen range of optimal regularization parameters yield, in general, a good correlation with the well data (the left column of Figure 4.6). The exceptions are solutions for $\lambda=0.1$ and $\lambda=0.2$, that are situated outside the red zone in Figure 4.6 and were included only for the sake of completeness. A good correlation was obtained even though the impedance models have blocky structures. The only exception was the part in the range of TWT 849-894 ms which was not modeled explicitly. We allow this error to occur since those layers are outside the clinoform sequence. In addition, the results for the given range of $\lambda$ yield a good match between the lithofacies log and the seismic trace, except for the above-mentioned part (the right column of Figure 4.6).

The resulting vertical resolution of this method is quite high and is better than the seismic resolution. From Figure 4.6 we can observe that layers with thicknesses down to $1 / 10^{\text {th }}$ of the source wavelength $(\approx 40 \mathrm{~m})$ are well resolved. This is substantially smaller than the classical resolution of one-quarter of the wavelength (Badley, 1985).

From the output of the inversion procedure we have enough confidence that the optimal regularization parameter is situated within the 
Stratigraphic model-based, low-parametrization seismic inversion

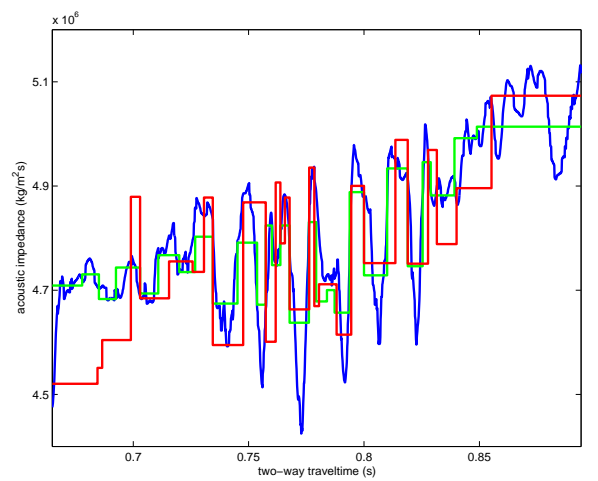

(a) $\lambda=0.1$

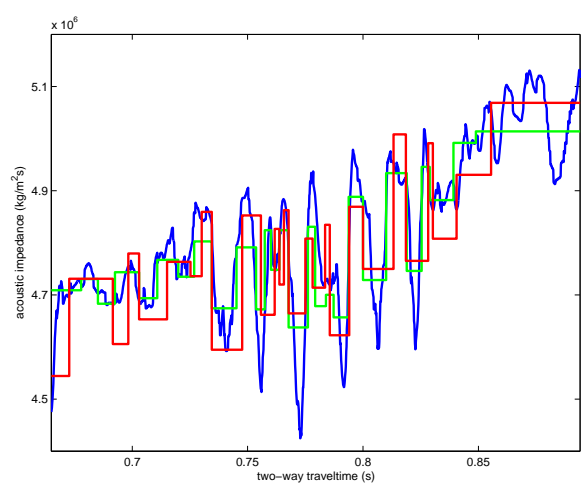

(c) $\lambda=0.2$

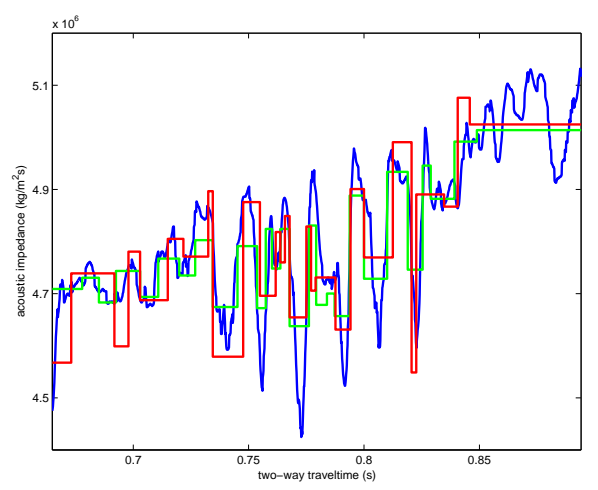

(e) $\lambda=0.3$

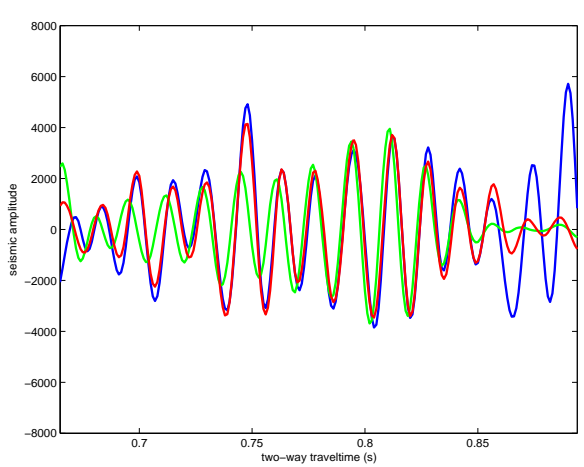

(b) $\lambda=0.1$

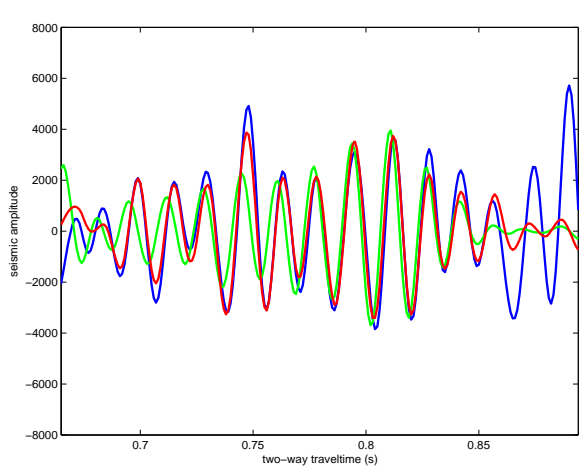

(d) $\lambda=0.2$

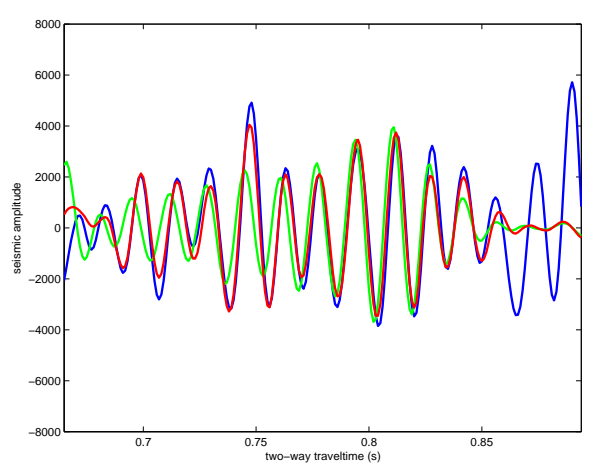

(f) $\lambda=0.3$ 


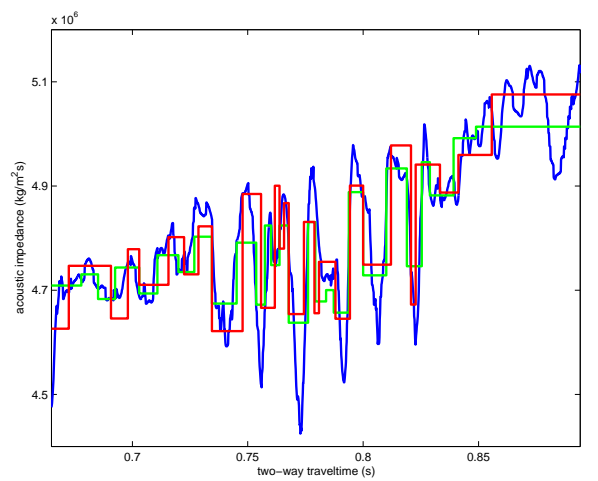

(g) $\lambda=0.4$

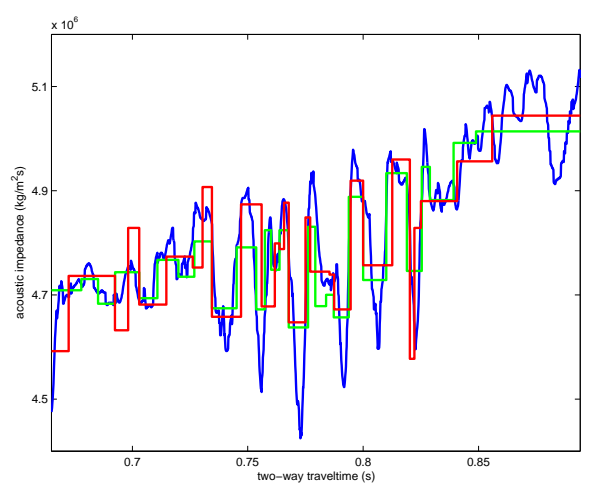

(i) $\lambda=0.5$

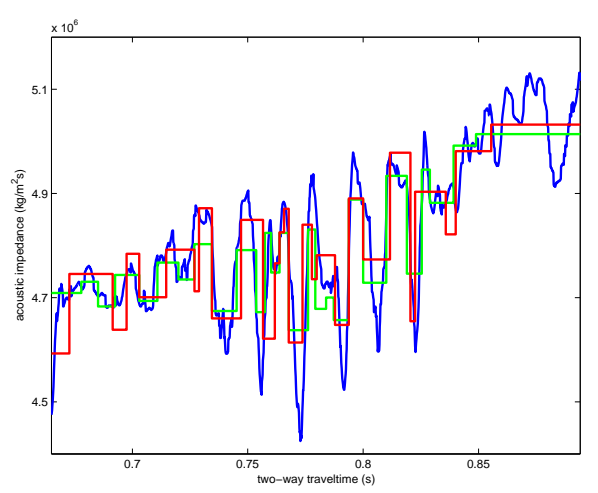

(k) $\lambda=0.6$

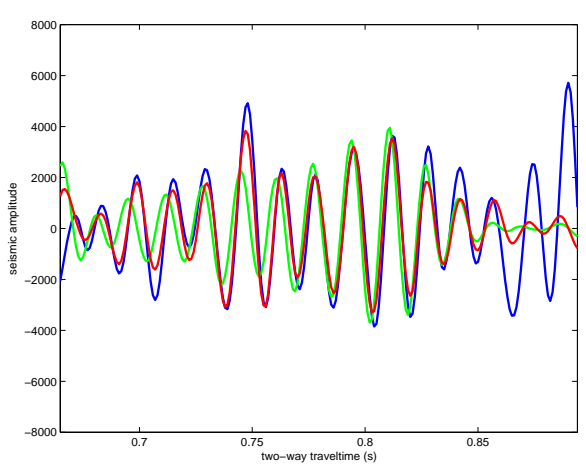

(h) $\lambda=0.4$

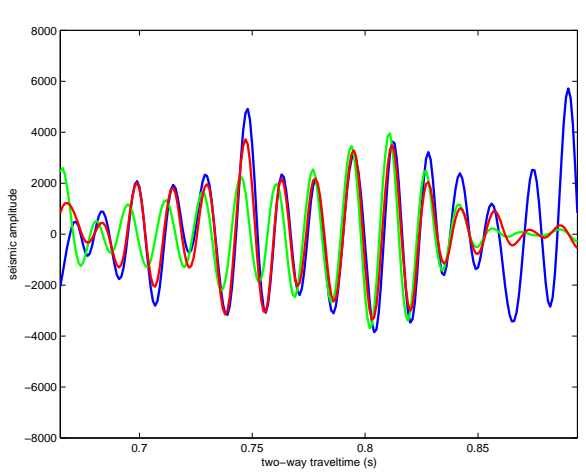

(j) $\lambda=0.5$

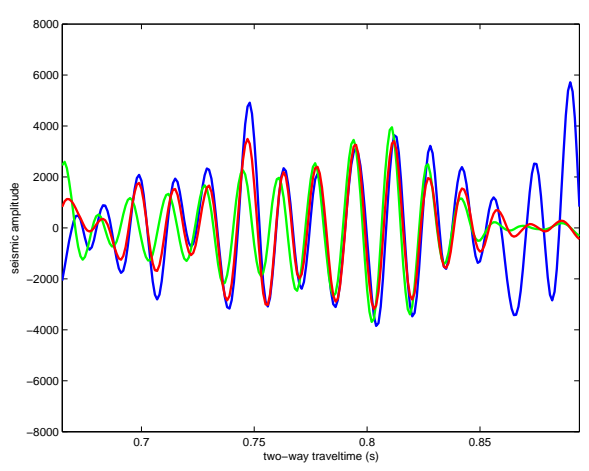

(l) $\lambda=0.6$ 
Stratigraphic model-based, low-parametrization seismic inversion

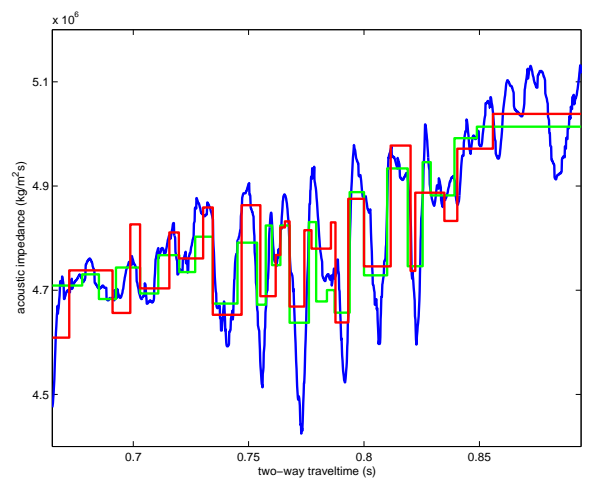

(m) $\lambda=0.7$

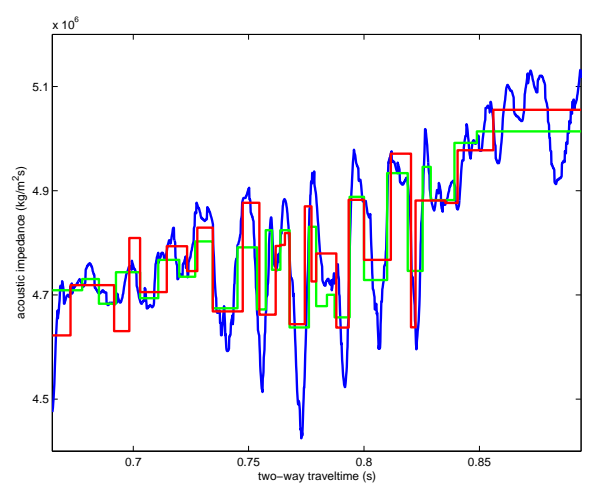

(o) $\lambda=0.8$

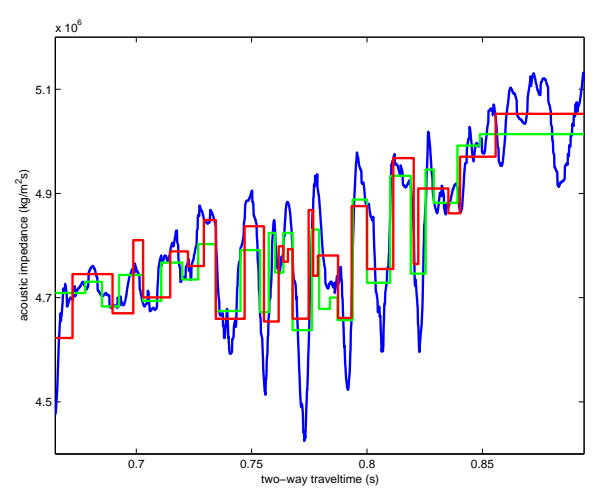

(q) $\lambda=0.9$

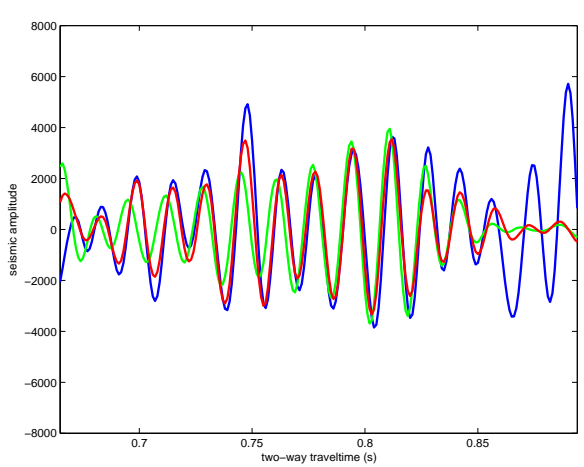

(n) $\lambda=0.7$

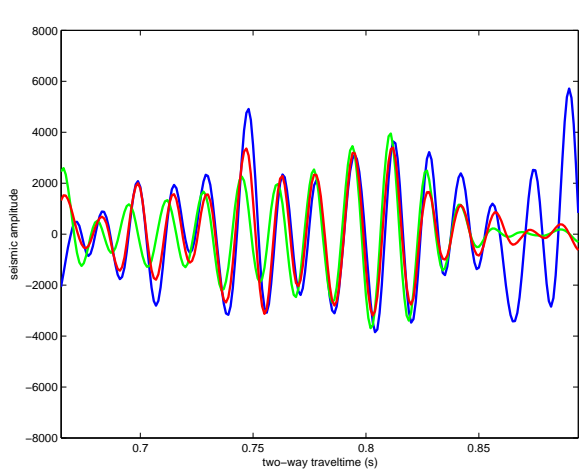

(p) $\lambda=0.8$

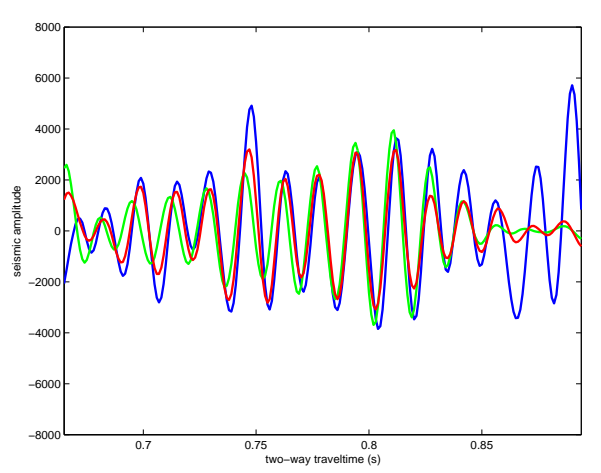

(r) $\lambda=0.9$ 


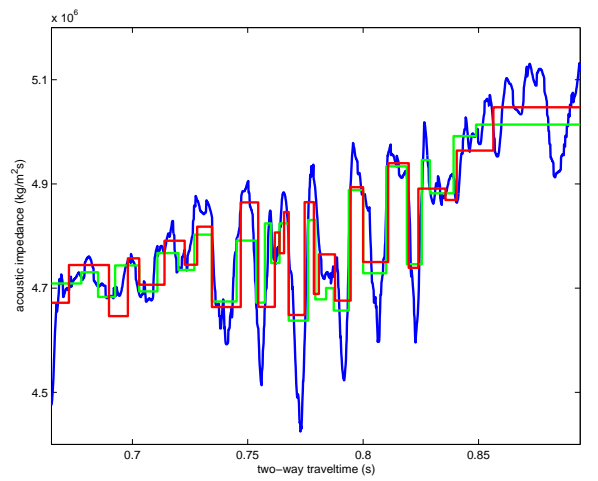

(s) $\lambda=1.0$

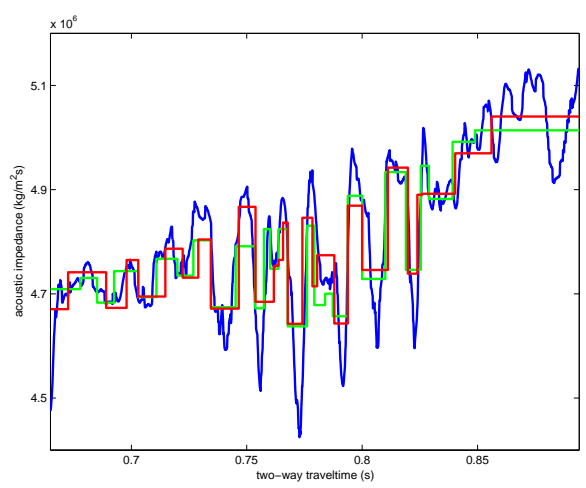

(u) $\lambda=1.1$

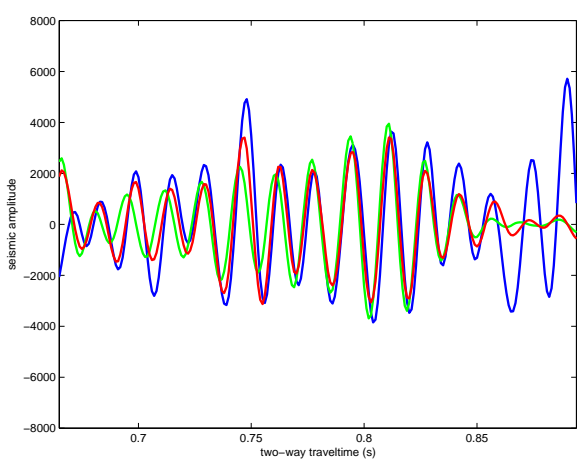

(t) $\lambda=1.0$

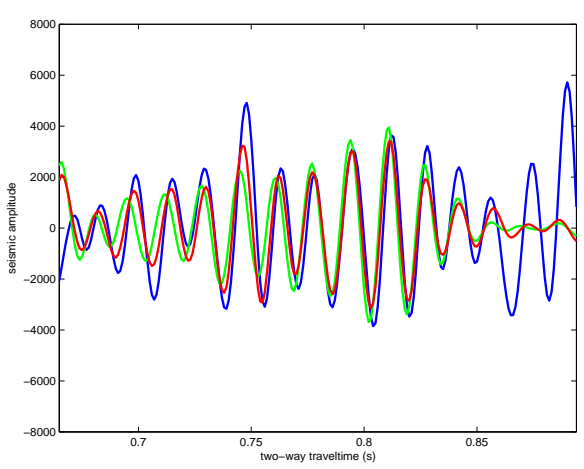

(v) $\lambda=1.1$

Figure 4.6: Results of $1 \mathrm{D}$ inversion for values of $\lambda$ in the interval [0.1 - 1.1]. The 'Well Model' (green), the estimated model (red), aligned on the actual impedance log (blue) (the left column) are displayed next to the synthetic seismic traces based on the 'Well Model' (green), the synthetic seismic based on the estimated model (red) and the measured seismic data (blue). 
Stratigraphic model-based, low-parametrization seismic

selected zone. The choice of the best $\lambda$ value based only on 1D inversion results remains difficult. In view of the fact that our main focus is the $2 \mathrm{D}$ clinoform sequence characterization we base the choice for the optimal value of the regularization parameter on the complex analysis of the $1 \mathrm{D}$ inversion results in combination with the pseudo $2 \mathrm{D}$ results (for the selected range of regularization parameters).

\subsection{Clinoform Geometry Estimation}

Once the parameters of the layer model (the acoustic impedance and layer time thickness) are estimated at the well location, the next step is to extrapolate this knowledge in the lateral direction along the clinoform package. For this, we first have to determine the exact clinoform shapes with the goal to parameterize them using a limited number of parameters. The set of parameters extracted can then be used to predict the presence of similar objects in this environment.

The advantage of using this method above a standard approach is the significantly smaller number of parameters that needs to be estimated. We use a small number of parameters to model the entire clinoform sequence that are later estimated in the inversion procedure instead of the standard way in which every point of the clinoform is shuffled. In addition, the outcome may serve as a predictive tool for the areas where seismic resolution is too low to distinguish objects in the seismic image.

\subsubsection{Geological Model}

The F3 data set offers a good opportunity to develop models for highresolution characterization of clinoform sequences because it contains a large-scale bedding in the clinoform parts of the sequence that converge 
to sub-seismic scale in the top- and bottom-set parts (Figure 3.15 and Figure 3.16).

\subsubsection{Mathematical Model}

Our goal is to describe a clinoform by a limited number of parameters. From a geometrical point of view a clinoform sequence can be roughly approximated by a set of translated sigmoidal curves. A sigmoid function $f_{j}(X)$ can be described by four parameters:

$$
f_{j}(X)=c_{j}+\frac{b_{j}}{1+e^{-\left(X-d_{j}\right) / a_{j}}},
$$

where $a_{j}$ is a lateral scaling, $b_{j}$ is a depth scaling, $c_{j}$ is a depth offset, $d_{j}$ is a lateral translation and the index $j$ identifies the clinoform number in a clinoform sequence.

Figure 4.7 illustrates the sigmoid curve transformation for two successive curves.

\subsubsection{Formulation of the inverse problem}

A next step is to relate the mathematical parameters of the sigmoid with relevant geological processes. This relationship will serve as a basis for clinoform system modeling. Based on an examination of the clinoform sequence, progradation can be associated with the lateral translation parameter and aggradation with the depth offset (Figure 3.3). We assume that geological objects are not random structures, but that they follow certain typical patterns caused by the depositional processes that formed them. Therefore, we can presume that for every subsequent clinoform within a fluvio-deltaic system, the lateral scaling and the depth scaling parameters, stay approximately constant, whereas the depth offset (aggradation) fluctuate a bit and the lateral 
Stratigraphic model-based, low-parametrization seismic

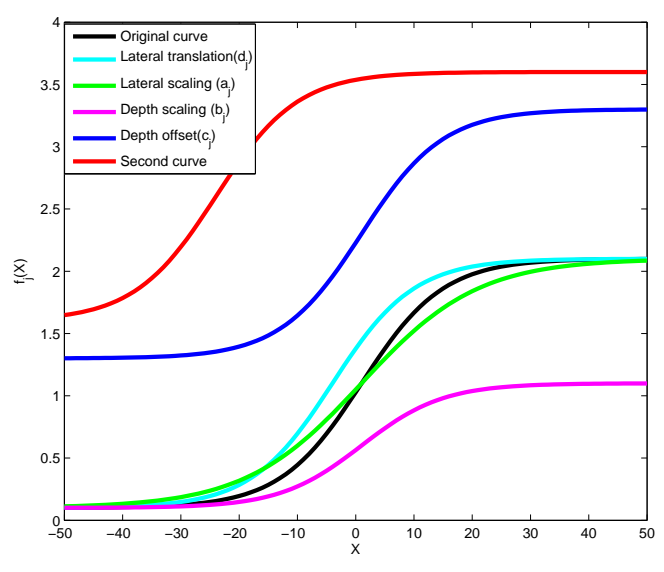

Figure 4.7: The dependence of the sigmoid curves on the scaling parameters.

translation (progradation) is strongly varying. As a result, the whole clinoform sequence can be modeled by means of just a few parameters. The parameter vector to be estimated is constructed by consecutive series of constant parameters ( $a$ and $b$ ) followed by varying parameters $\left(c_{j}\right.$ and $\left.d_{j}\right)$ for every curve.

$$
\boldsymbol{x}=\left[a, b, c_{1}, \ldots, c_{J}, d_{1}, \ldots, d_{J}\right],
$$

where $J$ is a number of clinoforms.

\subsubsection{Optimization}

The seismic objects extracted from the Unit 2 data set are partially eroded or otherwise affected by post-depositional processes. In addition, at the topsets and bottomsets the layers are thin and often poorly resolved on seismic images, leading to tuning effects. Refining the cli- 
noform structures is performed by model fitting. The objective (cost) function to be minimized is represented by the sum of the individual objective functions for every clinoform in the package. Each objective function is represented by a likelihood function only, due to absence of data independent a priori information.

\subsubsection{Initial Model}

An initial model of the parameters describing the sigmoid functions of the sequence was estimated from the seismic section by visual inspection with regard to the geological model of the sequence. Special attention was paid to the lateral translation parameter $d_{j}$ which shows the largest variations from one clinoform to the next. We introduced a lateral shift $\Delta d_{j}$ to the initial model $\boldsymbol{x}_{\mathbf{0}}$ for every subsequent curve, where $\Delta d_{j}$ is equal to a small fraction of the length of the clinoform

$$
\boldsymbol{x}_{\mathbf{0}}=[a^{0}, b^{0}, \underbrace{c^{0}, \ldots, c^{0}}_{J}, d^{0}, d^{0}+\Delta d_{1}, \ldots, d^{0}+\Delta d_{J-1}] .
$$

\subsubsection{Results}

The sigmoid model was fitted to four clinoforms $(J=4)$ within the entire sequence. The results are depicted in Figure 4.8 (dashed line), together with a semi-automated pick from the seismic section (solid lines). It can be seen that the clinoform shapes were estimated quite accurately. This supports our choice to model the clinoforms by a sigmoid function.

The results were superimposed on the seismic image in Figure 4.9 (the color scheme is kept the same) where the two main geological processes (progradation and aggradation) are very well observed. The quantative measurements for these processes are summarized in the Table 4.2. It can be seen that progradation is by far the most dominant 
Stratigraphic model-based, low-parametrization seismic

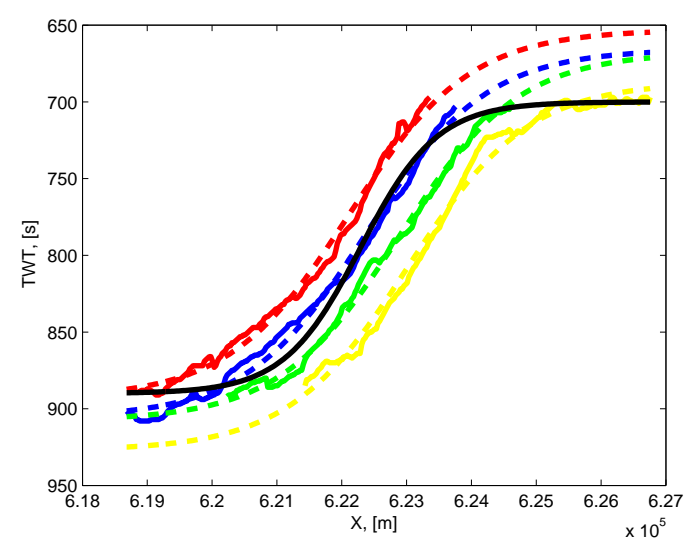

Figure 4.8: The estimated sigmoids of the four (partly eroded) clinoform curves (dashed lines), together with semi-automated picks from the seismic section (solid lines). The black line denotes the initial model.

\begin{tabular}{|c|c|c|c|}
\hline $\begin{array}{l}\text { Lateral } \\
\text { scaling }\end{array}$ & $\begin{array}{c}\text { Value } \\
(\mathrm{m})\end{array}$ & $\begin{array}{c}\text { Depth } \\
\text { scaling }\end{array}$ & $\begin{array}{c}\text { Value } \\
(\mathrm{m})\end{array}$ \\
\hline$a$ & $9 \overline{930}$ & $b$ & 240 \\
\hline \multicolumn{2}{|c|}{ Aggradation } & \multicolumn{2}{|c|}{ Progradation } \\
\hline $\begin{array}{l}\text { Depth } \\
\text { offset }\end{array}$ & $\begin{array}{c}\text { Value } \\
(\mathrm{m})\end{array}$ & $\begin{array}{c}\text { Lateral } \\
\text { translation }\end{array}$ & $\begin{array}{l}\text { Value } \\
(\mathrm{m})\end{array}$ \\
\hline$c_{1}$ (red curve) & 650 & $d_{1}$ & 0 \\
\hline$c_{2}$ (blue curve) & 670 & $d_{2}$ & 260 \\
\hline$c_{3}$ (green curve) & 670 & $d_{3}$ & 770 \\
\hline$c_{4}$ (orange curve) & 690 & $d_{4}$ & 910 \\
\hline
\end{tabular}

Table 4.2: Measurement of the progradation and the aggradation rates resulting from the fitting of sigmoids to the clinoforms. 


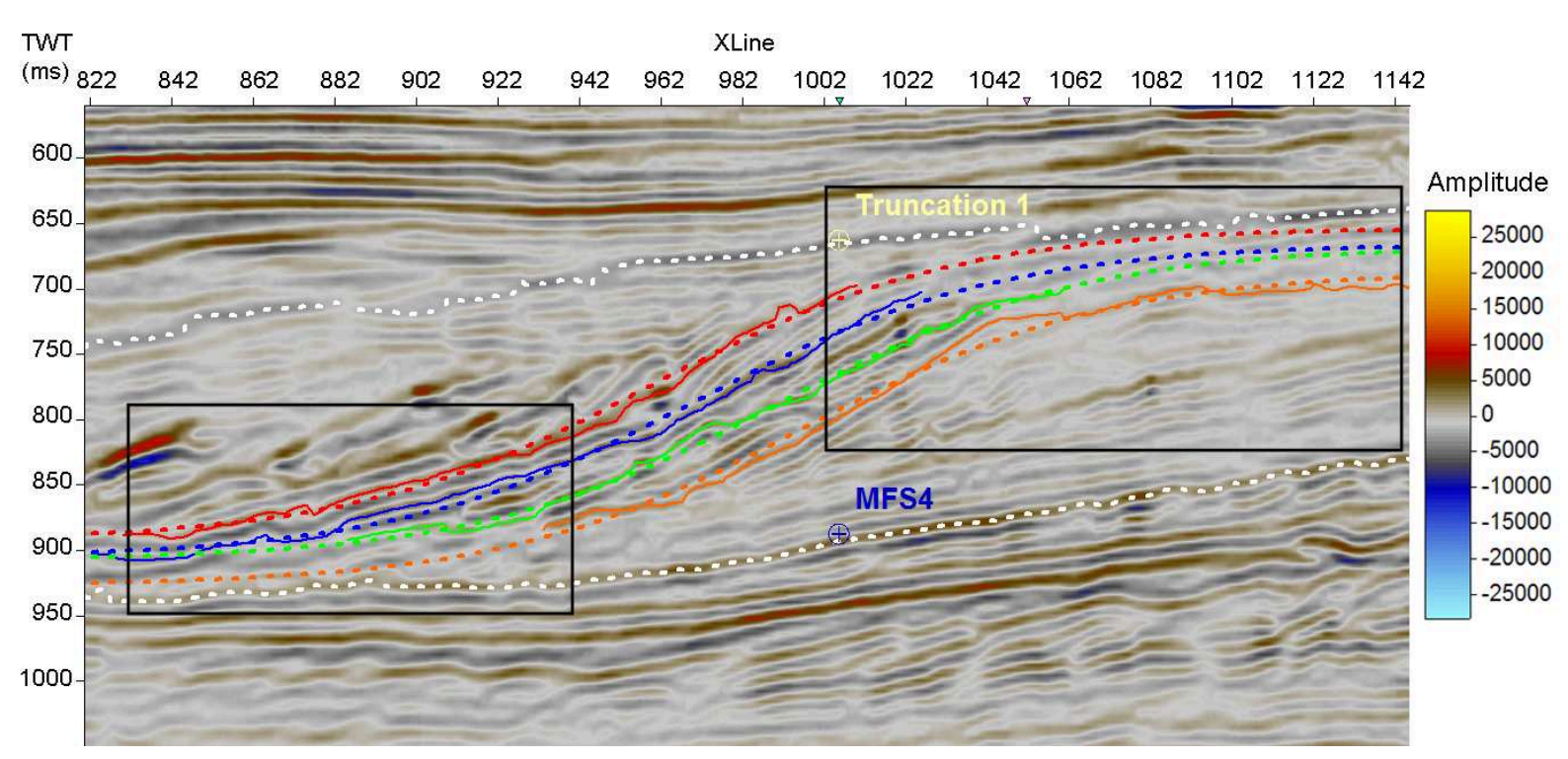

Figure 4.9: The same curves as in Figure 4.8, superimposed onto the seismic section. Scales and axes as in similar previous figures. Black rectangles refer to Figures 4.11 and 4.10. 
Stratigraphic model-based, low-parametrization seismic

process in this sequence, with aggradation proceeding at a very steady rate.

There are seismic events of parts of the geological layers that are at subseismic scale, particularly at the topset and bottomset of the sequence, and cannot be tracked on the seismic image. The acoustic impedance contrast is very low between the layers in those areas, making boundary identification nearly impossible from the seismic image. The modelled sigmoids can trace the layers from the clinoform part to these regions and serve as guides to characterize these sub-seismic layers.

Enlarged versions of the bottomset and the topset of the clinoform layers are depicted on Figure 4.10 and Figure 4.11, respectively. The black arrows indicate areas where the clinoform layers converge to an extent such that inversion is only possible through guidance by the sigmoidal model (dashed lines).

In addition, the estimated depths of the four clinoform curves (for which the colors are kept the same as in previous figures) are superimposed onto well F03-04 (Figure 4.12).

\subsection{Inversion of the Clinoforms}

\subsubsection{Formulation of the inverse problem}

In our approach, the inversion of a seismic section is done trace by trace. The technique is the same for each trace and is described in the previous section. The parameters to be estimated for every trace are the acoustic impedances and the time thicknesses of the layers

$$
\boldsymbol{x}_{k}=\left[Z_{k, 1}, \ldots, Z_{k, N}, T_{k, 1}, \ldots, T_{k, N}\right]
$$




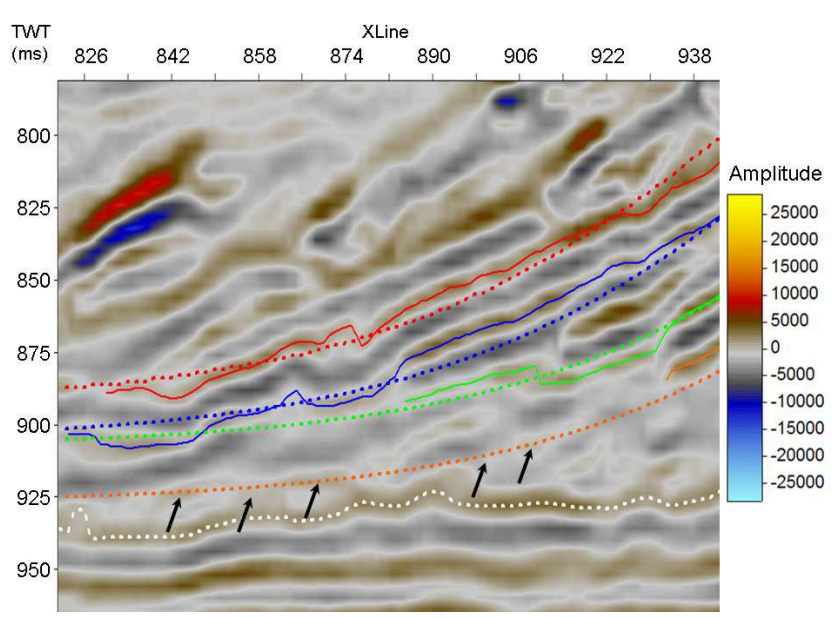

Figure 4.10: Enlargement of the left black rectangle in Figure 4.9, with the black arrows indicating areas of strong convergence of the reflectors. For discussion see text.

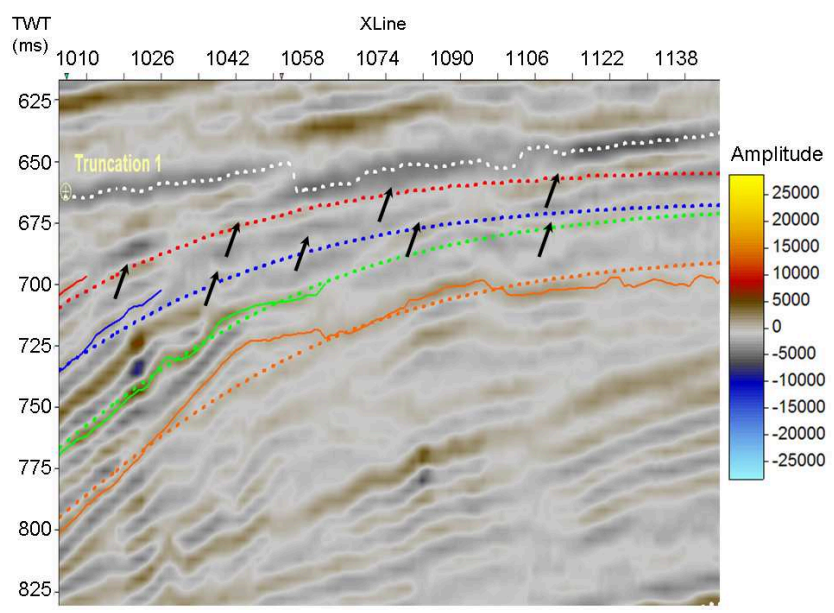

Figure 4.11: Enlargement of the right black rectangle in Figure 4.9, with the black arrows indicating areas of strong convergence of the reflectors. For discussion see text. 


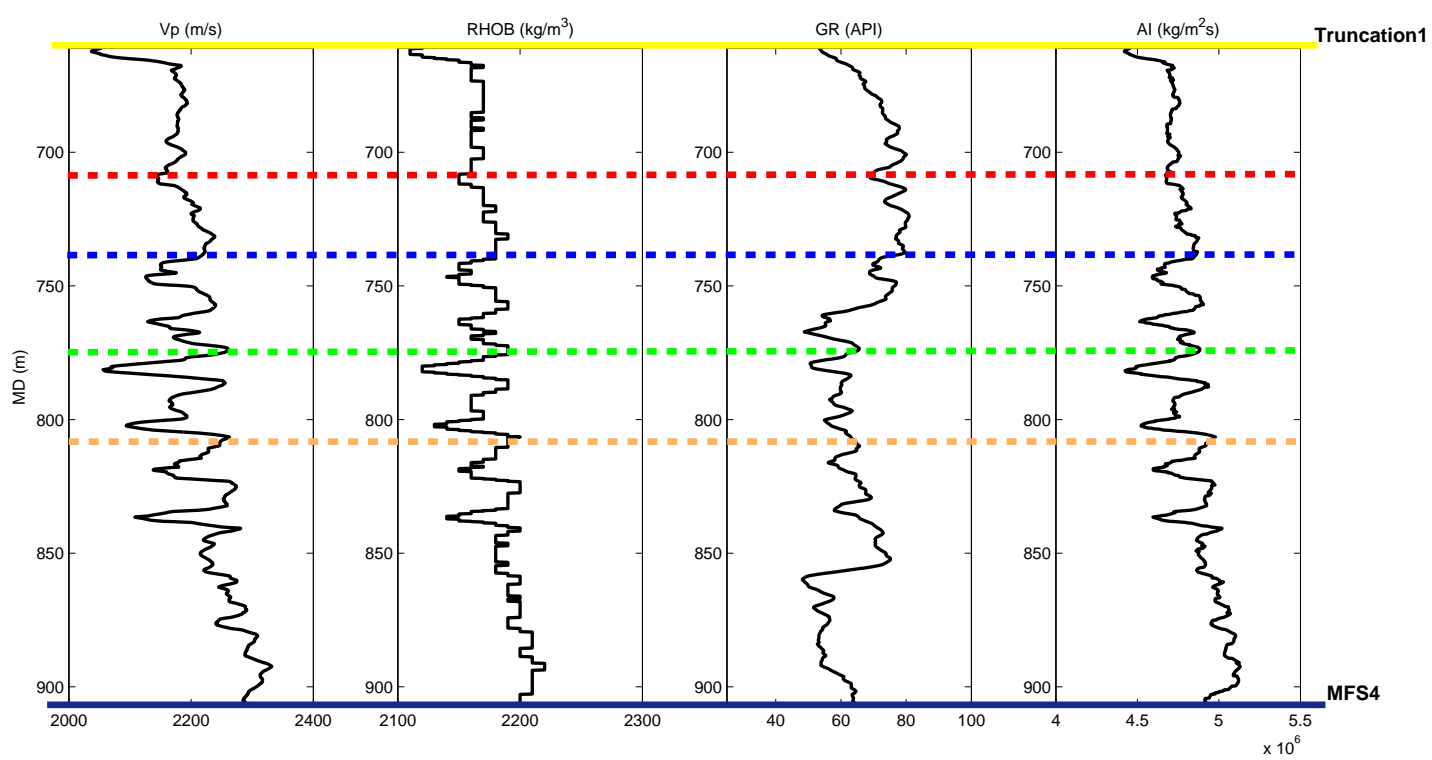

Figure 4.12: The three well logs and the computed acoustic impedance log in well F03-04, together with the depths at which the computed sigmoidal clinforms occur. Same colors as in Figure 4.9. 'Truncation 1' (yellow) and 'MFS4' (blue) indicate the top and bottom of the clinoform package. 
where $N=27$ is the number of layers, $k=1, \ldots, K$, where $K$ is the number of traces in the sequence to be inverted. A seismic section containing $K=70$ traces, situated on the left-hand side of the well, was chosen for the test (Figures 3.14 and 3.15). Analogously to the 1D case, the well log data are used as a priori information.

\subsubsection{Structural Information Incorporation}

A central issue of this method is the incorporation of geological information into the inversion process. The structural information which we integrate is a mathematical description of the clinoform shapes. These shapes are used to steer the trace inversion along the sequence, i.e., they serve as hard constraints (two-way travel time horizons vs $X$-coordinates).

The inversion process starts from the trace closest to the well. In this case the 'Well Model' can again serve as an initial model.

For the sake of convenience we assume that the number of layers (defined in the 'Well Model') between two hard boundaries (estimated clinoform horizons) is kept constant throughout the entire sequence. Although this may be a questionable assumption we can vary the number of layers using the technique presented in Chapter 5 .

Once the first trace is inverted, then the result (estimated parameters) can be used as an initial model for the next trace. In addition, structural information (retrieved from the estimated clinoform shapes) provides knowledge about the trace-to-trace variation. Since the time thickness between estimated clinoform shapes - the hard constraints - varies from trace to trace, a normalization (stretch or compress) factor is applied to adjust the initial model to the next trace. The normalization factor is defined by the ratio of the time lapse between the estimated shapes (hard constraint) of the current 


\section{Stratigraphic model-based, low-parametrization seismic}

seismic trace (to be inverted) and the previous seismic trace (just inverted).

\subsubsection{Regularization}

The range of the possible values for the regularization parameters $\lambda$ was selected based on the analysis of the 'L-curve' for the 1D inversion. In Figure 4.13 we present the pseudo 2D inversion results for the selected zone. The left column demonstrates the estimated acoustic impedance models of the clinoform sequence, while the right column, provides the synthetic seismic responses of the impedance models.

\subsubsection{Results}

Unfortunately, in practice, when data always contain noise and the forward model is oversimplified, there is no technique which is capable to find the optimal value of the regularization parameter for a non-linear inverse problem. The aforementioned 'L-curve' method only helps to isolate a set of possible optimal solutions. Since there is no objective way of determining the optimal regularization parameter from the selected group, a subjective approach can be applied afterwards. A subjective approach can for example be based on the expertise of a geologist, who can choose the most realistic solution from a geological perspective. Of course, it is important to analyze both the $1 \mathrm{D}$ and pseudo $2 \mathrm{D}$ results, since the influence of a priori information on the inversion process decreases with the distance to the well. 1D inversion results for a selected set of the regularization values $\lambda$ are shown in Figure 4.6; Figure 4.13 shows pseudo 2D inversion results.

From a geological point of view, the 1D inversion as well as the pseudo $2 \mathrm{D}$ results for the value of $\lambda=0.3$ appear to be the most realistic. The estimated impedance model for $\lambda=0.3$ is shown in Figure 4.14(a). 


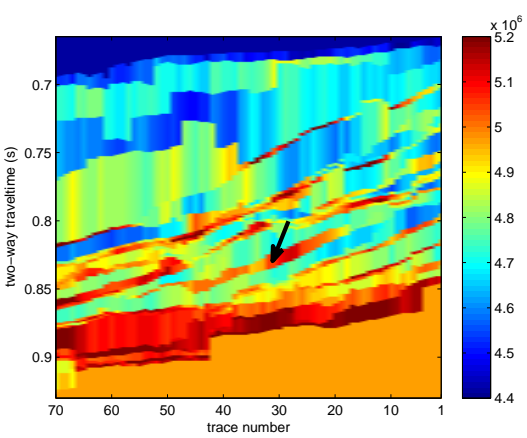

(a) $\lambda=0.1$

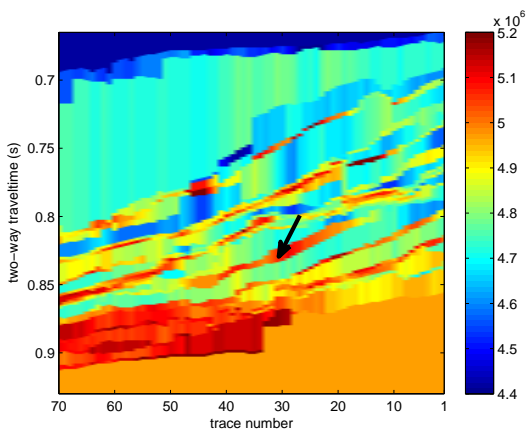

(c) $\lambda=0.2$

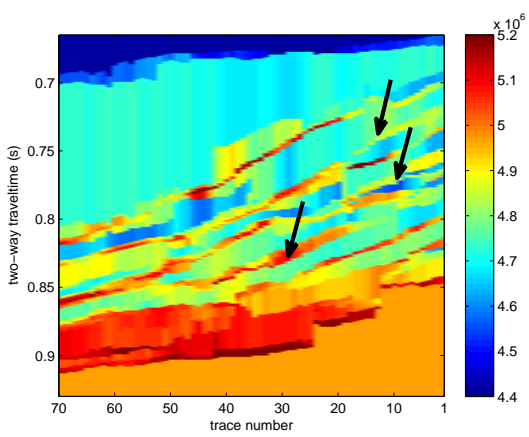

(e) $\lambda=0.3$

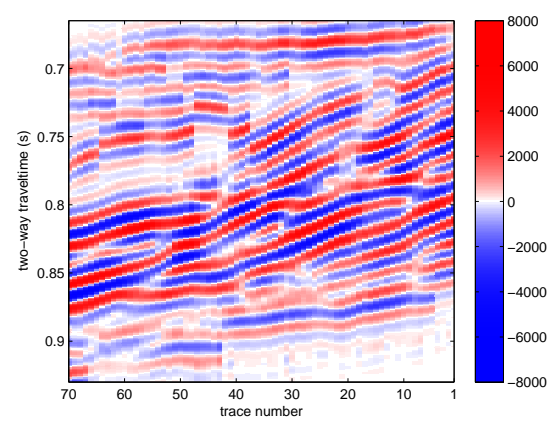

(b) $\lambda=0.1$

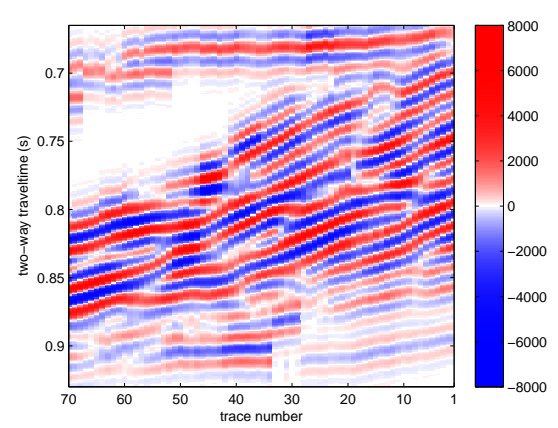

(d) $\lambda=0.2$

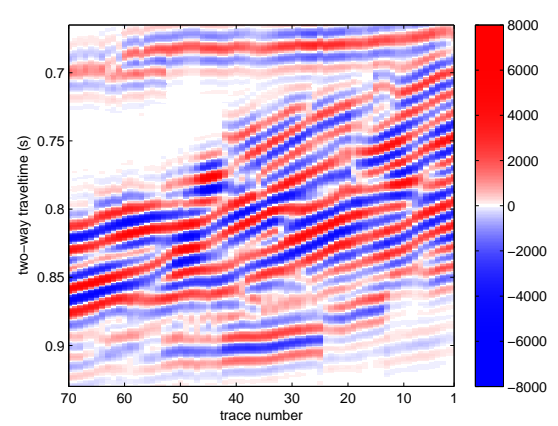

(f) $\lambda=0.3$ 
Stratigraphic model-based, low-parametrization seismic

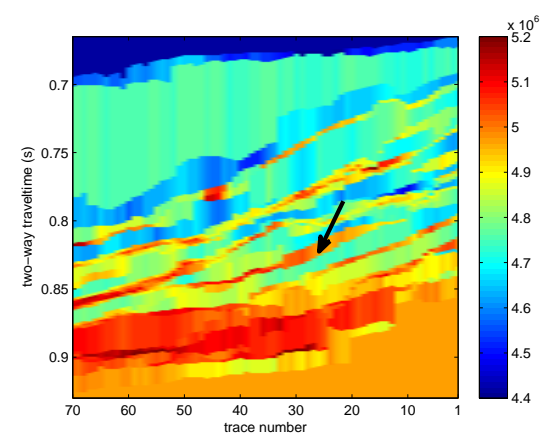

(g) $\lambda=0.4$

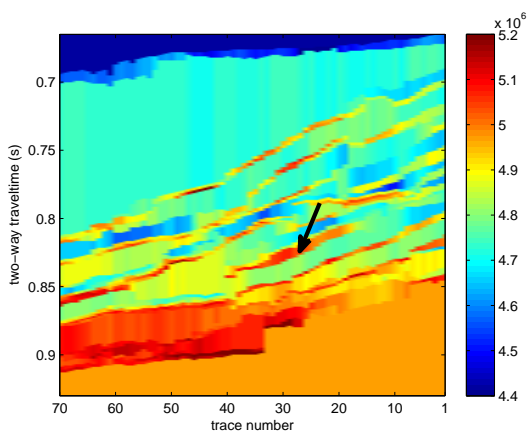

(i) $\lambda=0.5$

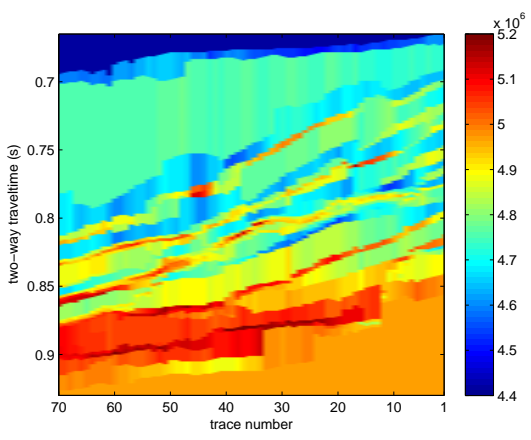

(k) $\lambda=0.6$

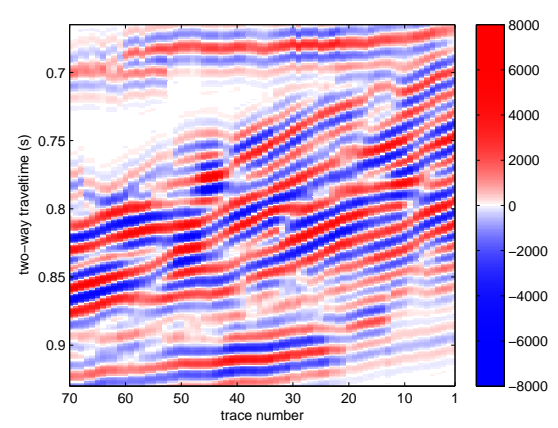

(h) $\lambda=0.4$

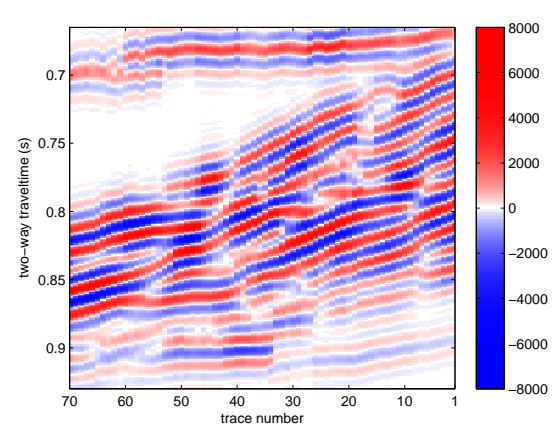

(j) $\lambda=0.5$

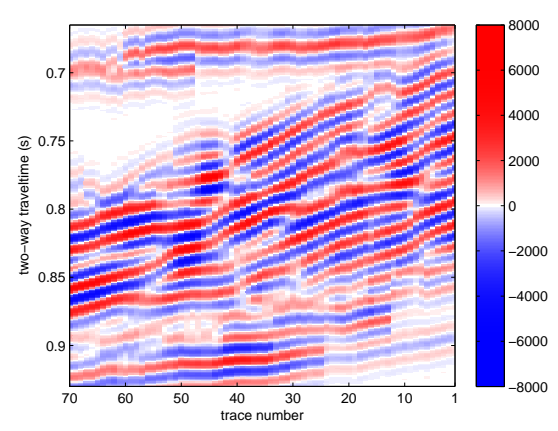

(l) $\lambda=0.6$ 


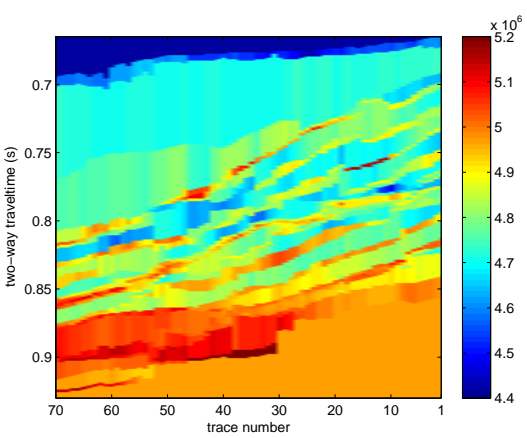

(m) $\lambda=0.7$

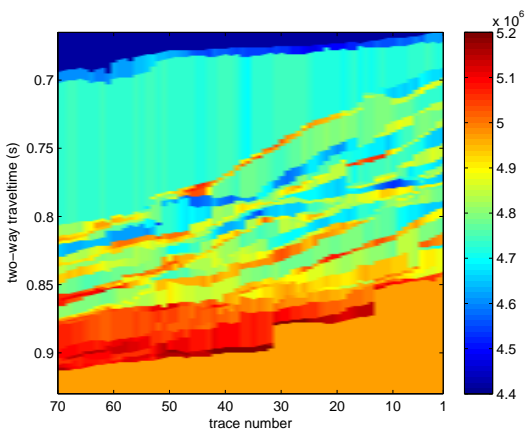

(o) $\lambda=0.8$

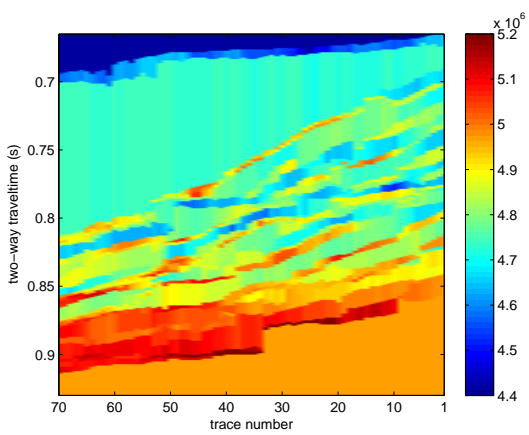

(q) $\lambda=0.9$

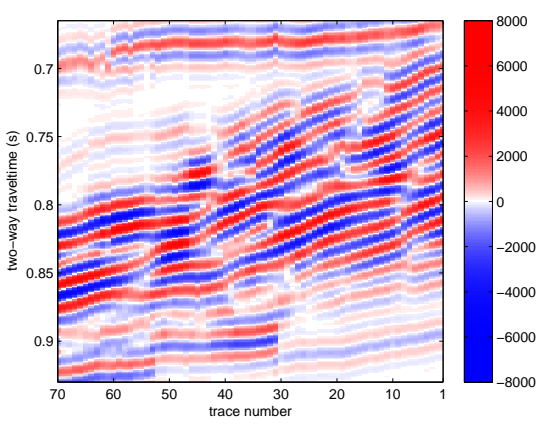

(n) $\lambda=0.7$

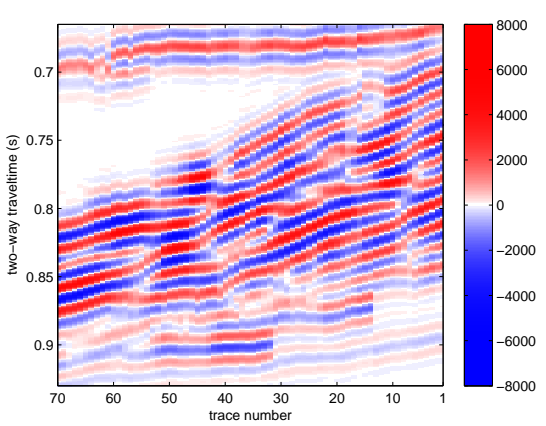

(p) $\lambda=0.8$

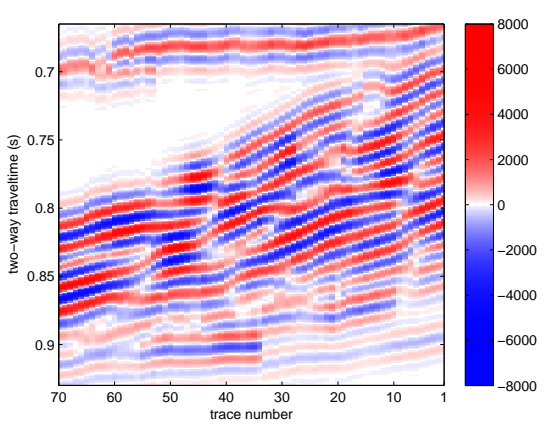

(r) $\lambda=0.9$ 
Stratigraphic model-based, low-parametrization seismic

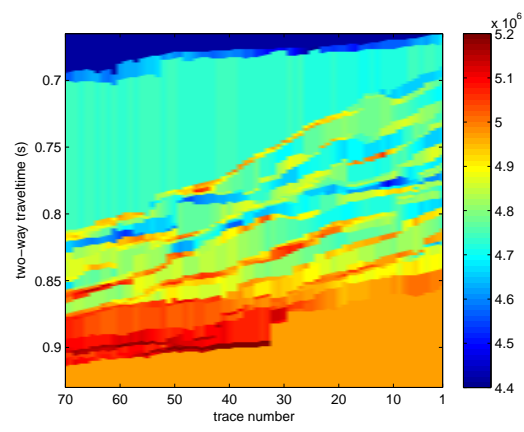

(s) $\lambda=1.0$

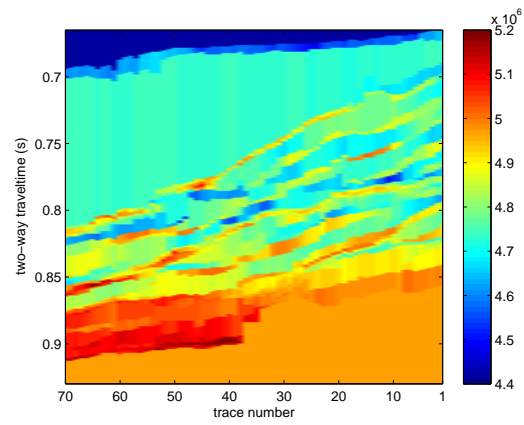

(u) $\lambda=1.1$

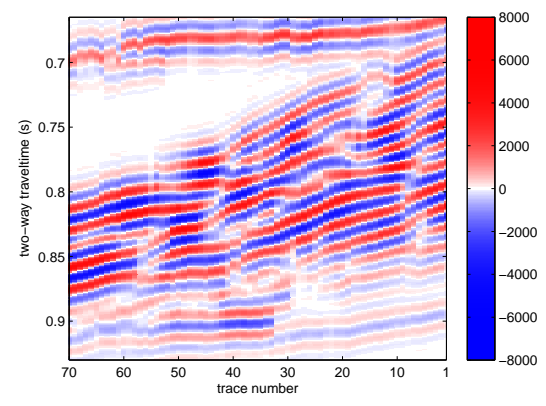

(t) $\lambda=1.0$

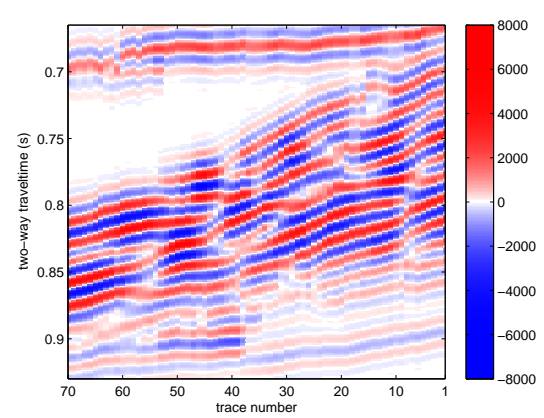

(v) $\lambda=1.1$

Figure 4.13: Results of pseudo 2D inversion of the clinoform section for values of $\lambda$ in the interval $[0.1-1.1]$. The estimated impedance models (left column) are displayed together with the synthetic seismic (right column). The black arrows indicate those areas where the layers seen on the seismic image (Figure 4.14(c)) are well observed on the impedance model. 
The model reveals that the internal layers of the clinoform sequence gently follow the estimated clinoform shapes. Some layers appear or disappear in the middle of the section and follow the sequence while on the right hand side layers overlap each other. At the same time, the properties of the layers are slowly changing in the lateral direction, driven by seismic data. The black arrows indicate the areas of interest, where the details(layers) that are present on the actual seismic image (Figure 4.14(c)) are well observed on the impedance model.

The simulated seismic sections computed from the impedance model of Figure 4.14(a) is displayed in 4.14(b). The figures illustrate that the method gives a good match with the field seismic, but it has to be kept in mind that the blank areas were not modeled explicitly since they fall outside the clinoform sequence (the integration of the structural information was performed between clinoform shapes only). For comparison, the field seismic is displayed in Figure 4.14(d) and Figure 4.14(c).

The resulting impedance models for higher values of $\lambda$ were found to reveal too much influence of the a priori information. High $\lambda$ values do not allow the properties (acoustic impedances as well as time thicknesses) to deviate much from the well data. As a consequence the impedance models expose that properties do not change much in the lateral directions. On the contrary, low values of $\lambda$ give too much freedom for properties to change in the lateral direction but deviate significantly from the well data. As a result, the lateral continuity of the impedance models for those $\lambda$ values is quite poor. In conclusion, the synthetic seismic for both the lower and the higher $\lambda$ values show a slightly worse correspondence to the actual seismic than the selected optimal one. 
Stratigraphic model-based, low-parametrization seismic

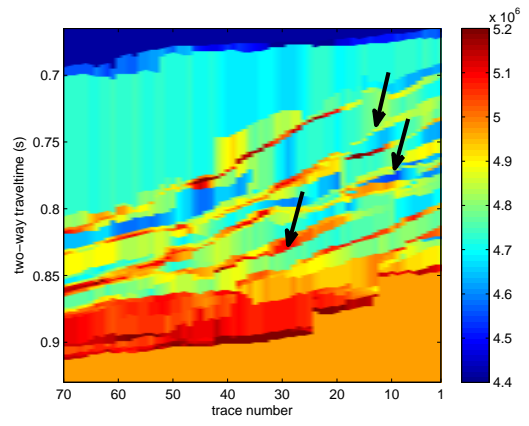

(a)

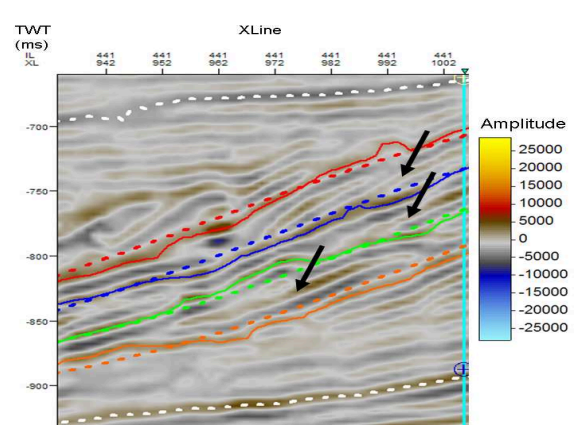

(c)

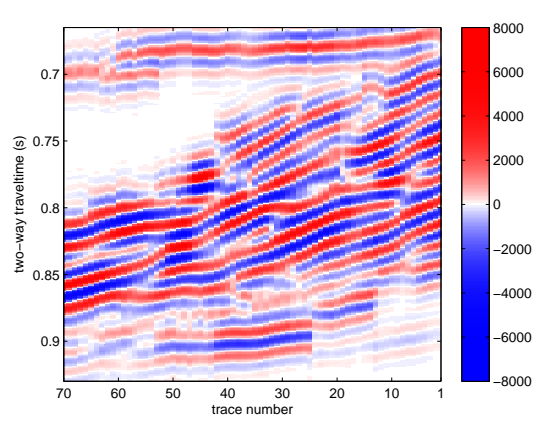

(b)

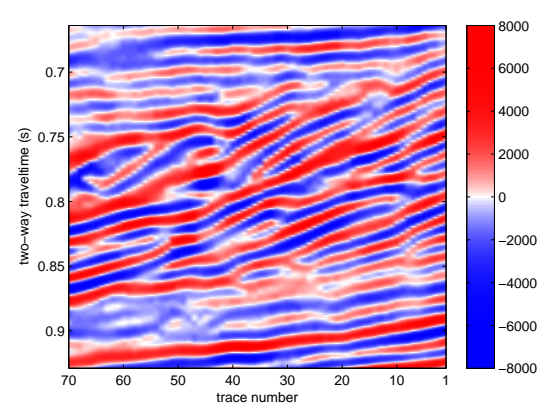

(d)

Figure 4.14: The pseudo-2D inversion results for the optimum regularization with $\lambda=0.3$ : (a) the impedance model of the clinoform sequence; (b) the simulated seismic section based on the estimated impedance model; (c) the actual seismic section in the target zone with the interpreted clinoform layers; and (d) the actual seismic section in the same color scheme as (b). Scale and axes as in similar previous figures. 


\section{Chapter 5}

\section{Grid-based Seismic Inversion}

\section{$5.1 \quad$ Introduction}

The assumption made in the model-based inversion of the previous chapter regarding a constant number of layers in the 'Well Model' is a serious limitation because that approach does not support layers to (re)appear/disappear along the clinoform sequence. Observation of the seismic data set showed that this assumption is valid in some parts of the sequence but not in others. The method introduced in this chapter overcomes this problem by abandoning the explicit layer model of the previous chapter. Again a trace-by-trace inversion is applied, starting from the well, to characterize the 2D clinoform sequence. All acquisition parameters (including the extracted wavelet) are kept constant w.r.t. the method in Chapter 4. The well data remains as a source of a priori information, but in a completely different representation.

To enforce a sparse solution we adopted a method by Van Eekeren et al. (2008) that was successfully applied to obtain 'super-resolution' 
reconstruction of small moving objects from a low-resolution undersampled image sequence.

This method differs from the well-known sparse spike acoustic inversion algorithms (Pendrel and van Riel, 1997; Debeye and van Riel, 1999) in the employed sparsity promoting factor. Instead of commonly used techniques such as $L_{p}$ deconvolution (Debeye and van Riel, 1999), mixed norms methods (Alliney and Ruzinsky, 1994), Cauchy criterion (Amundsen, 1991), Sech criterion (Crase et al., 1990), Huber criterion (Huber, 1981), etc, we employed a Total Variation (TV) criterion, which is a modified $L_{1}$-norm (Rudin et al., 1992; Li and Santosa, 1996; Farsiu et al., 2004). The norm provides excellent constraints (a priori knowledge) for problems with sparse solutions.

This method uses the same data misfit term as in equation (2.17), but employs regularization terms that favor sparse solutions, i.e., solutions for which $\|\nabla \boldsymbol{x}\| \cong 0$ holds for the majority of samples. The regularization within one trace is performed by the so-called vertical operator. This vertical operator favors a sparse inversion solution within the trace. In addition to the vertical operator we also introduce a so-called horizontal operator that favors continuity of the pseudo $2 \mathrm{D}$ inversion results and supports propagation of the well data along the sequence.

\subsection{Mathematical Background}

\subsubsection{Super Resolution Reconstruction of Small Moving Objects}

Recently, Van Eekeren et al. (2008) introduced a method for multiframe super-resolution (SR) reconstruction of small moving objects 
(i.e., objects that consist solely of boundary pixels). This method is used to improve the visibility, detection as well as automatic recognition of small moving objects. This approach simultaneously estimates a sub-pixel precise polygon boundary as well as a high-resolution intensity description of a small moving object. The method is split into three parts:

1. Constructing a background model and detecting the moving object,

2. Fitting a model-based trajectory for object registration,

3. Obtaining a high-resolution object description, containing a subpixel accurate boundary and a high-resolution intensity description, by solving an inverse problem.

The method attracted our attention since the last step (3.) shows some resemblance to our inverse problem. The cost function of the inversion method, apart from the data misfit, includes a term which regularizes the amount of intensity variation within the object according to a criterion similar to the Total Variation (TV) criterion (Li and Santosa, 1996).

\subsubsection{Total Variation Criterion}

One of the most successful regularization methods for denoising and deblurring is the total variation (TV) method (Rudin et al., 1992; Li and Santosa, 1996; Farsiu et al., 2004). The TV criterion penalizes the total amount of change in the image as measured by the $L_{1}$-norm of the magnitude of the gradient and is defined as

$$
\Upsilon_{T V}(x)=\|\nabla x\|_{1}
$$


where $\nabla$ is the gradient operator. The most useful property of the TV criterion is that it tends to preserve edges but suppresses numerous small fluctuations in the reconstruction. The small fluctuations are often a result of the ill-posedness and noise. The use of an $L_{1}$-norm for this purpose is crucial. An $L_{2}$-norm as in Tikhonov regularization does not work, because relatively small fluctuations in $x$ are not penalized sufficiently. Following this argument would lead to the use of an $L_{\alpha^{-}}$ norm with $\alpha$ in the interval $[0,1]$. However, choosing $\alpha$ smaller than 1 , yields a non-convex problem which cannot be solved by the current state-of-the-art optimization techniques.

\subsubsection{Optimization}

The minimization is done in an iterative way using the LevenbergMarquardt method. The cost function in the framework of this method has a form like $\min _{\beta} \sum_{i}^{N}\left(\chi-\chi_{i}(\beta)\right)^{2}$, where $\chi$ is the measurement and $\chi_{i}(\beta)$ is the estimate depending on parameter $\beta$. The method assumes that the cost function has first and second order derivatives that exist everywhere. However, the $L_{1}$-norm does not satisfy this assumption.

\section{Hyperbolic Norm}

To be able to apply the Levenberg-Marquardt method in combination with an $L_{1}$-norm we use the hyperbolic norm $\left(\|\bullet\|_{H}\right)$ introduced by Van Eekeren et al. (2008). This norm is defined as

$$
\|x\|_{H}=\sum\left(\sqrt{x_{i}^{2}+\alpha^{2}}-\alpha\right) .
$$

The hyperbolic norm has the same properties as the $L_{1}$-norm for large values $\left(x_{i} \gg \alpha\right)$ and it has first and second derivatives that exist everywhere. 


\subsection{D Inversion of the Clinoforms}

The goal of the method is similar to that of the previous chapter, namely to get a structural image of the clinoform sequence by identifying its main layer structure and properties.

\subsubsection{Formulation of the Inverse Problem}

There are several approaches to the subject of forward modeling and a priori information incorporation that were successfully implemented in the previous chapter and thus are employed here as well:

- The basic one-dimensional convolution given in equation (2.4) is used as forward model.

- The wavelet extracted from the real data set (Chapter 3, Figure 3.18) serves as a source wavelet.

- The well data serves as a source of priori information.

A significant difference between this method and the previous one is that instead of creating an initial well model for 1D inversion based on lithofacies analysis of the well logs (method described in the previous chapter), the logs (impedance in our case) are resampled with a constant step and used as an initial model instead.

\section{Sampling}

The sampling of the well data can be performed either in the spatial domain or in the time domain. Sampling in the spatial domain may appear more straightforward since the log data are measured in the spatial domain (depth) as well. However, sampling in the time domain is more convenient in case the post-stack seismic data are the 
time-migrated data. The convenience comes from the fact that the sampling step can be related to the desired details that can be detected from the resulting estimated impedance models.

The current inversion method is tested on the North Sea seismic data set, which is sampled as a function of two-way travel time. Hence the vertical resolution is defined in the time domain. As a result, the log data were re-sampled in the time domain, with a constant time step $T$ (equation (4.1)). This procedure performed by averaging the log values (in our case, the values of the impedances) within each time sample $T_{j}$ in the target zone. Then, the initial model $\boldsymbol{x}_{0}$ based on the log sampling is as follows:

$$
\boldsymbol{x}_{0}=\left[Z_{1}^{0}, \ldots, Z_{M}^{0}\right],
$$

where $M$ is the number of samples per trace. Consequently, the parameter vector $\boldsymbol{x}$ to be estimated is constructed by the acoustic impedances $Z$ only, since $T_{j}=T$ is set constant

$$
\boldsymbol{x}=\left[Z_{1}, \ldots, Z_{M}\right] .
$$

The vertical time window to which the inversion of the previous chapter was applied, is bounded by two fixed boundaries, namely the top and the bottom of the target Unit 2. The current method is different in the sense that the inversion is carried out in a predetermined time window. Taking into account the propagation of the clinoform in the horizontal direction, the vertical time window for the analysis was set from 650 to $950 \mathrm{~ms}$.

To decide on a proper sampling, the impedance log (only the part which belongs to the predetermined time window) was resampled with a range of different time steps $T$. By proper sampling we understand 
a sampling which sets a balance between keeping the number of parameters to be estimated $(M)$ relatively low (equation (5.4)) while maintaining a good resemblance between the initial model and the original log.

To keep the number of parameters to be estimated relatively low, $T=4 \mathrm{~ms}$ was chosen to be the maximum time step. On the other hand, $T=0.5 \mathrm{~ms}$ was chosen as the minimum time step to achieve more accurate sampling of the log. Two intermediate values, $T=2 \mathrm{~ms}$ and $T=1 \mathrm{~ms}$, were included in the range.

Figure 5.1 illustrates the sampled impedance log (green) of the initial model superimposed on the original impedance log (blue) for the selected range of time steps.

The initial model for a time step $T=4 \mathrm{~ms}(M=75)$ does not offer sufficient level of detail to faithfully represent the lithofacies. The log becomes too smooth and their layers are suppressed. On the other hand, the initial model for a time step $T=0.5 \mathrm{~ms}(M=600)$ samples the $\log$ with too much detail. Note that every detail remains visible. Both the initial models with time steps $T=2 \mathrm{~ms}(M=150)$ and $T=$ $1 \mathrm{~ms}(M=300)$ provide a good trade-off between the two conflicting requirements: a small number of parameters and a good resemblance with the original log.

\subsubsection{Vertical Operator}

As stated in the introduction, in the present method, the original cost function $F(\boldsymbol{x})$ is modified by including two additional terms. The first additional term is the sum of absolute differences between adjacent samples (gradient magnitudes) of the solution vector of the trace. For the sake of convenience, this is further referred to as the "vertical operator'. This term favors solutions in which the sum of gradient 


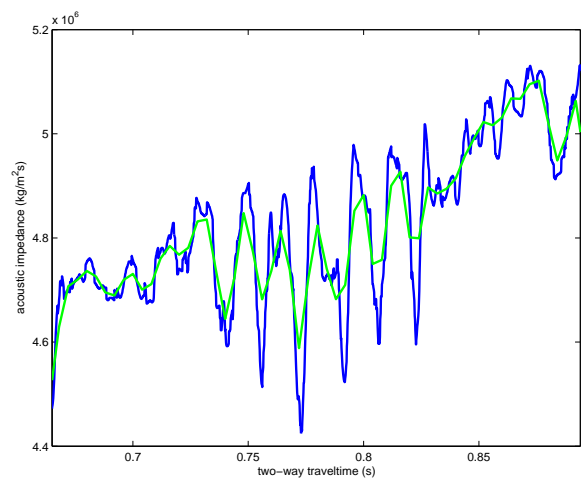

(a) $T=4 \mathrm{~ms}$

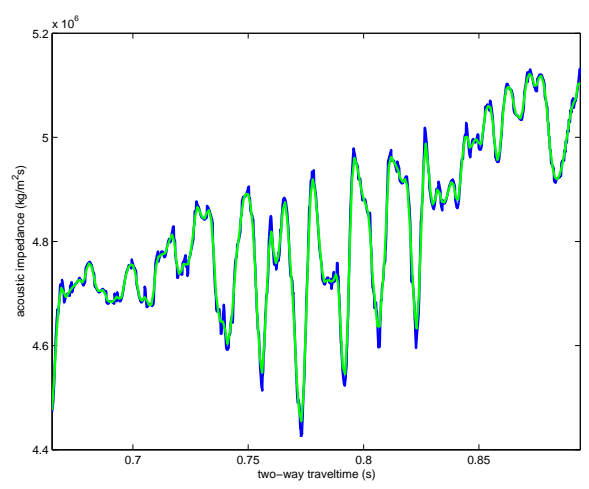

(c) $T=1 \mathrm{~ms}$

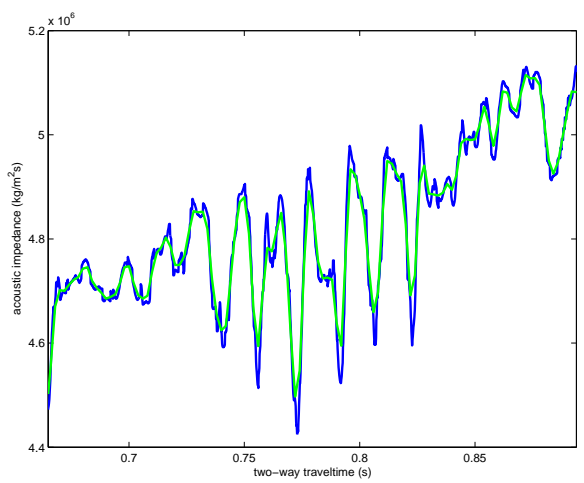

(b) $T=2 \mathrm{~ms}$

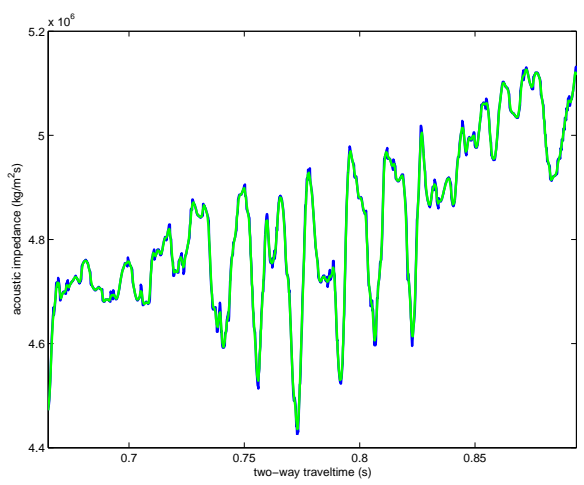

(d) $T=0.5 \mathrm{~ms}$

Figure 5.1: The sampled impedance log (green) of the initial model, superimposed on the original impedance log (blue) for time sampling with time steps of $T=4 \mathrm{~ms}, 2 \mathrm{~ms}, 1 \mathrm{~ms}$ and $0.5 \mathrm{~ms}$.

magnitudes is small; these are called 'sparse solutions'. It is well known that the $L_{1}$-norm is favoring a sparse solution. For our application the hyperbolic norm has a preference since it allows the LevenbergMarquardt optimization method to be used for minimization of our 
function. The values of acoustic impedances to be estimated $(\boldsymbol{x})$ are much larger than 1 , hence, by setting $\alpha$ equal to 1 the hyperbolic norm has the same properties as the $L_{1}$ norm. The hyperbolic norm also tries to minimize the number of inverted layers by penalizing absolute differences between adjacent samples. Layers are no longer modeled explicitly, but must show up in the resulting trace as a result of the fact that the output of adjacent samples should be similar, except at layer boundaries. Based on the TV criterion, the trace (the log and the acoustic impedance model) sampling should be such that we have many samples inside a single layer, i.e., the number of layers is much smaller than the number of samples $M$ per trace. The model parameters are found by minimizing

$$
\begin{aligned}
F(\boldsymbol{x})=(\boldsymbol{y}-\boldsymbol{g}(\boldsymbol{x}))^{T} \boldsymbol{C}_{n}^{-1}(\boldsymbol{y}-\boldsymbol{g}(\boldsymbol{x}))+ \\
+\lambda\left(\boldsymbol{x}-\boldsymbol{x}_{i}\right)^{T} \boldsymbol{C}_{x}^{-1}\left(\boldsymbol{x}-\boldsymbol{x}_{i}\right)+ \\
\quad+\mu \sum_{j}\left(\sqrt{\left(x_{j}-x_{j-1}\right)^{2}+1}-1\right),
\end{aligned}
$$

where $j$ is a sample index and $\boldsymbol{x}$ are the acoustic impedances (stored in a vector) we are looking for. It was shown in Chapter 4, that when applied to our case, the covariance matrix from equation (2.11) transforms to a scalar (equation (4.4)). For the sake of convenience, the last term in equation (5.5) is normalized with the same factor $\sigma_{\boldsymbol{x}_{\boldsymbol{i}}}^{2}$ (equation (4.4)).

\section{Number of Identified Layers}

To get an idea of the number of layers in a trace after minimizing $F(\boldsymbol{x})$ (equation (5.5)), the following method was proposed. As described above, the layering is controlled by the vertical operator that favors sparse solutions, meaning that acoustic impedances that have 
similar properties are clustered together, thereby forming a layer. Consequently, the layering shows up as series of maxima and minima of the estimated impedance curve. Estimating the amount of layers therefore comes down to counting the number of maxima and minima within the desired time interval (Figure 5.2). This can easily be counted by counting the number of sign changes in the first derivative of the estimated impedance curve. However, the method is quite sensitive to small layer fluctuations. A well-known method to regularize the differentiation operator is to apply it in scale space. Effectively this corresponds to replacing the derivative operator by a convolution with the derivative of a Gaussian function. The scale of the Gaussian (i.e., its standard deviation) can now be used to suppress small fluctuations.

\subsubsection{Regularization}

Minimization of a cost function, including the modified version in this chapter, is an ill-posed problem, and therefore regularization needs to be applied. In the original cost function (equation (2.17)), the parameter $\lambda$ balances the data misfit and the difference between the a priori information and solution misfit. In the modified version of the cost function (Equation 5.5), the parameter $\lambda$ preserves its original meaning but does not regulate the balance anymore, as the function $F(\boldsymbol{x})$ contains the additional term, the so-called 'vertical operator'. The weighting coefficient $\mu$ in front of the vertical operator term restricts the amount of layers in the inverted trace. Moreover, it penalizes fluctuations inside each layer.

A central issue in this section is how to select the weighting coefficients $\lambda$ and $\mu$ in front of the terms. As indicated in Chapter 4, there is no technique which is capable to find the optimal value of the regularization parameter for non-linear inverse problems in practice. Instead, the tool called 'L-curve' method was proposed, that helped to isolate a 


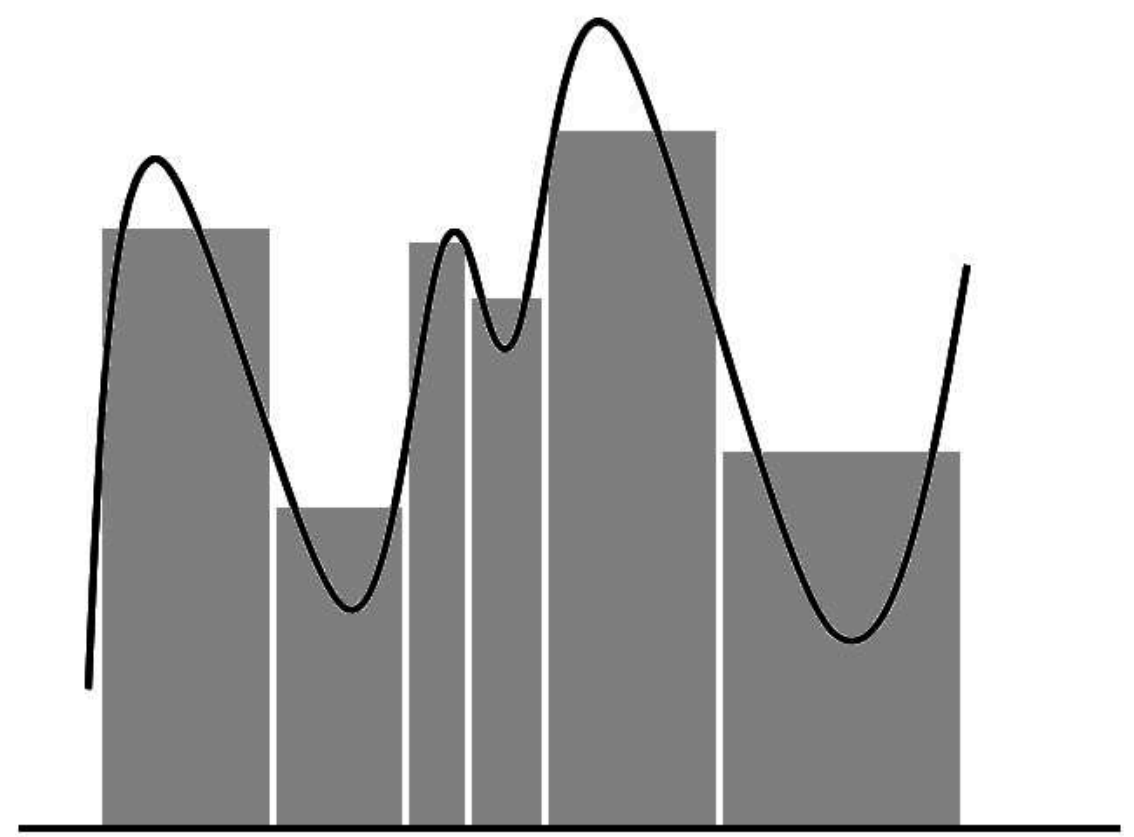

Figure 5.2: The sketch showing how the number of layers (the gray blocks) in the resulting acoustic impedance model (solid black line) is estimated.

set of possible optimal solutions. Clearly, in case of the modified cost function (equation (5.5)), even the 'L-curve' method does not seem to work. In this case, the only potential way to select an optimal regularization is empirical.

The understanding of the physical background of the weighting coefficients and their relationships helps steering the search. Before the 
optimal regularization can be found, tests on the sensitivity of the parameters are necessary. The tests are expected to provide information regarding dependence between the parameters.

Taking into account the role of the covariance matrices $\boldsymbol{C}_{\boldsymbol{x}}$ (equation (4.4)) and $\boldsymbol{C}_{\boldsymbol{n}}$ (equation (4.5)), the selection of the $\lambda$ values for the test was driven by common sense. The values $\lambda=0.5, \lambda=1$ and $\lambda=2$ were selected and are expected to exhibit the low and the relatively high influence of the a priori term.

Due to its different nature, a broader range of values form $\mu$ were tested: $\mu=0.5, \mu=1, \mu=2, \mu=4, \mu=8$. We expect that this range of $\mu$ values is able to demonstrate the effect of restricting the number of layers in an acoustic impedance model.

The last parameter, that does not show up explicitly in equation (5.5) but which is playing an important role, is the sampling step $T$ (equation (4.1)) and associated with it, the number of samples $M$ (parameters to be estimated, equation (5.4)). Based on the outcome of the analysis of the sampling rates (Figure 5.1), $T=2 \mathrm{~ms}$ and $T=1 \mathrm{~ms}$ values were selected for the test.

For convenience, the values of the all parameters for the sensitivity tests are summarized in Table 5.1. The sensitivity tests for each combination of parameters $(\lambda, \mu, T)$ were performed separately.

The outcome of the sensitivity test is a set of $1 \mathrm{D}$ inversion results of the seismic trace closest to the well. For convenience, the results for all tests are organized in a similar manner as Table 5.1, they are put in figures consisting of two columns. The left columns show the initial models (resampled impedance log) in green, the estimated models in red, superimposed on the actual impedance log in blue. The right column permits the comparison of several traces: the synthetic seismics 


\begin{tabular}{|c|c|c|c|c|c|c|c|c|c|c|c|}
\hline \multicolumn{6}{|c|}{ Sampling rate $T=2 \mathrm{~ms}$} & \multicolumn{6}{|c|}{ Sampling rate $T=1 \mathrm{~ms}$} \\
\hline$\lambda$ & \multicolumn{5}{|c|}{$\mu$} & $\lambda$ & \multicolumn{5}{|c|}{$\mu$} \\
\hline 0.5 & 0.5 & 1 & 2 & 4 & 8 & 0.5 & 0.5 & 1 & 2 & 4 & 8 \\
\hline 1 & 0.5 & 1 & 2 & 4 & 8 & 1 & 0.5 & 1 & 2 & 4 & 8 \\
\hline 2 & 0.5 & 1 & 2 & 4 & 8 & 2 & 0.5 & 1 & 2 & 4 & 8 \\
\hline
\end{tabular}

Table 5.1: Parameters in the 1D sensitivity tests.

based on the initial model (resampled impedance log) in green, the synthetic seismics in red and the actual seismic data in blue. For a systematic approach, the analysis of the test results is performed accordingly, i.e., first, the influence of the regularization parameters on the estimated acoustic impedance models is described followed by an analysis of the synthetic seismic traces.

$\lambda$ Sensitivity The influence of $\lambda$ on the result of $1 \mathrm{D}$ inversion of the clinoform package was examined for the given range of $\lambda=0.5, \lambda=1$ and $\lambda=2$. For the sake of consistency, the other two parameters were kept constant i.e., $\mu=2, T=2 \mathrm{~ms}$.

Figure 5.3 shows the outcome of the test. The left column illustrates that the estimation of the impedance model, with $\lambda$ increasing from 0.5 to 2 , is not much affected, except for the intervals of two way travel time (TWT) $0.65-0.7 \mathrm{~s}$ and $0.83-0.9 \mathrm{~s}$. The increase of $\lambda$ values gives slightly better results in these regions. These intervals are characterized by an alternation of thin layers with similar acoustic properties. A close look at the estimated impedance model at TWT $0.83 \mathrm{~s}$ in Figure 5.3(a) $(\lambda=0.5)$ reveals the presence of a single layer, while the same layer is being split into two separate thin layers for $\lambda=2$ (Figure $5.3(\mathrm{e}))$. This indicates that thin layers are mostly affected by the $\lambda$ parameter. 
The effect of $\lambda$ on the estimation of the corresponding seismic traces from the right column is relatively low. The comparison of Figure 5.3(b) and Figure 5.3(f) reveals that the mismatch between the synthetic seismic trace and the actual one is slightly higher when $\lambda$ increases.

What can be concluded from this test is that, although an effect of $\lambda$ regularization is observed, especially on thin layers, the influence of the $\lambda$ parameter, within the given range, on the results of the $1 \mathrm{D}$ inversion is not substantial.

$\boldsymbol{\mu}$ Sensitivity For the determined range of $\mu$ values $(\mu=0.5,1,2,4,8)$ a sensitivity test was performed. Following considerations similar to the previous case, the other two parameters were kept fixed $(\lambda=1$ and $T=2 \mathrm{~ms}$ ).

Figure 5.4 illustrates the results of the test. From the sequence of plots presented in Figure 5.4, an effect of the parameter $\mu$ on both the estimated impedance section and the synthetic seismic section can be clearly observed. As expected, increasing $\mu$ restricts the fluctuations

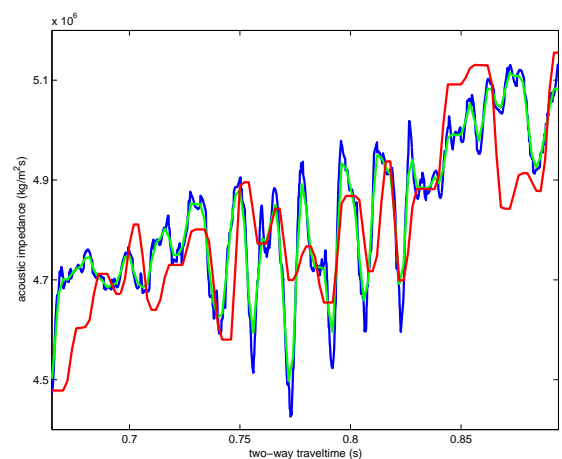

(a) $\lambda=0.5$

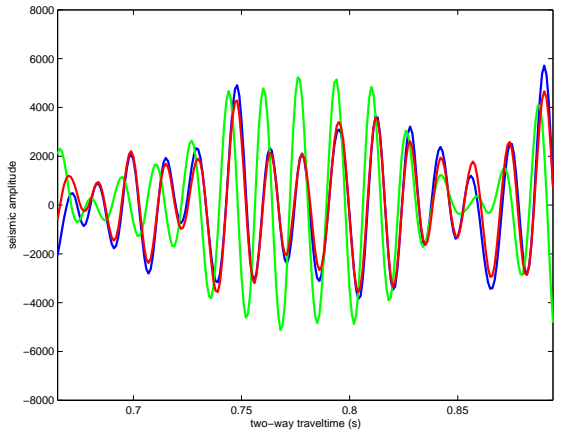

(b) $\lambda=0.5$ 


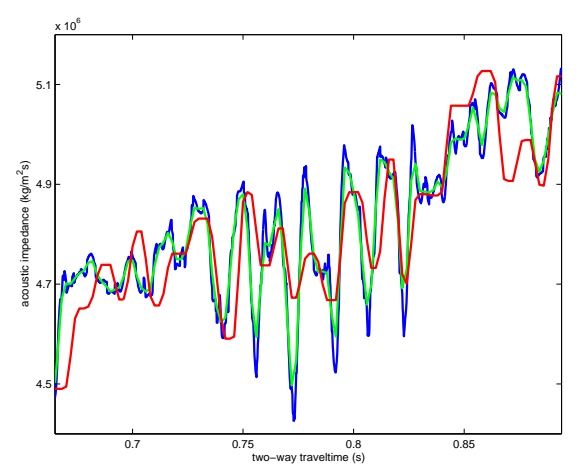

(c) $\lambda=1$

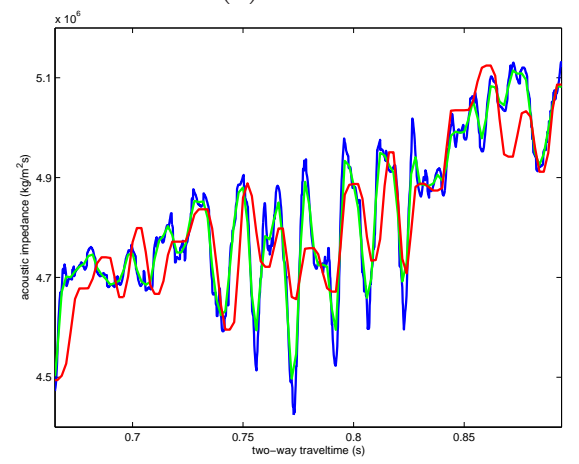

(e) $\lambda=2$

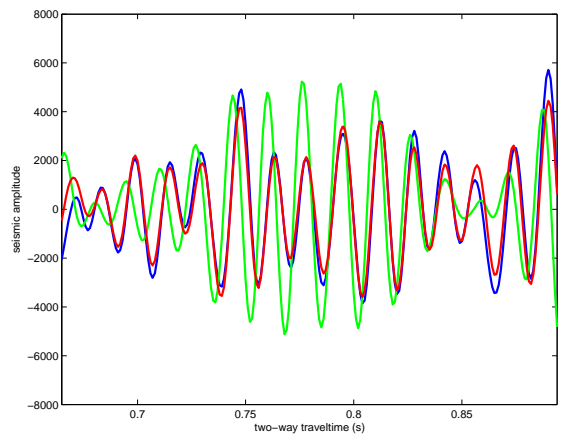

(d) $\lambda=1$

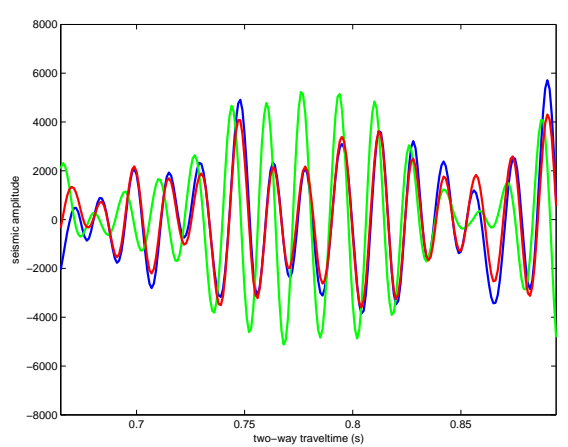

(f) $\lambda=2$

Figure 5.3: $\lambda$ sensitivity test $(\mu=2 ; \lambda=0.5,1,2 ; T=2 \mathrm{~ms}$; $M=150)$. Results of 1D Inversion of the Clinoforms. Left column: the initial model (resampled impedance log, green), the estimated model (red), superimposed on the actual impedance log (blue). Right column: seismic traces of the initial model (resampled impedance log) based synthetic seismic (green), the synthetic seismic (red) and the actual seismic data (blue). 


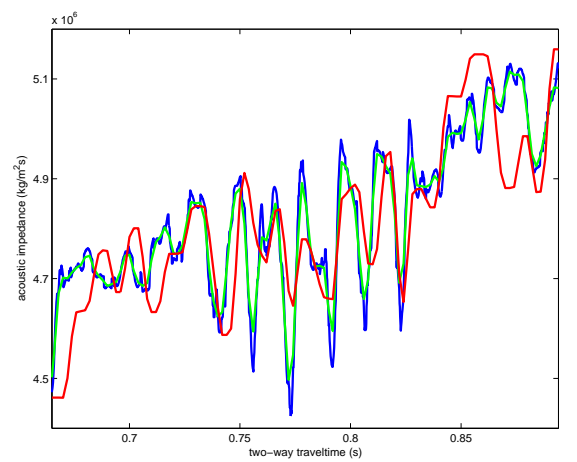

(a) $\mu=0.5$

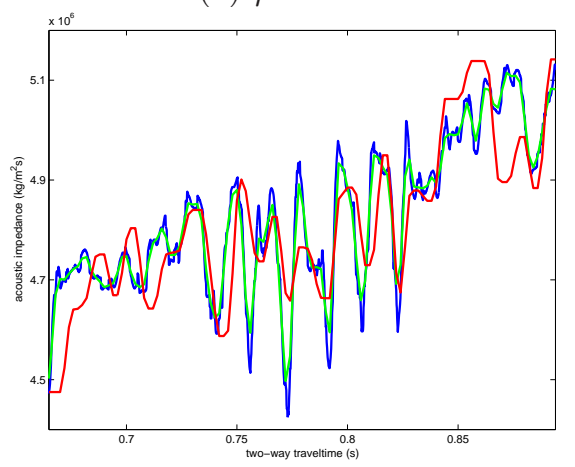

(c) $\mu=1$

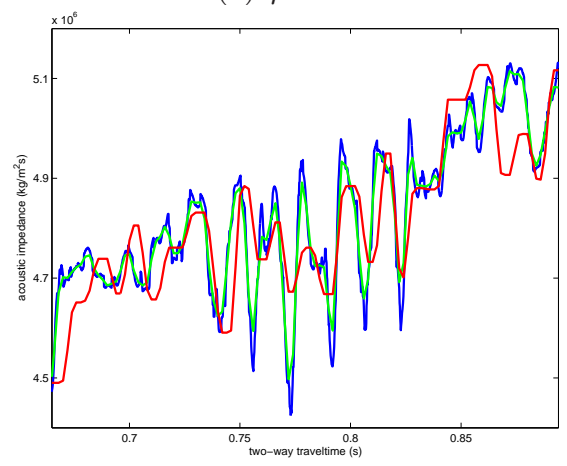

(e) $\mu=2$

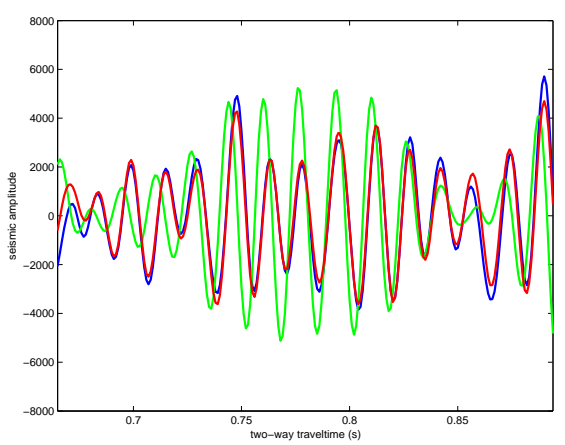

(b) $\mu=0.5$

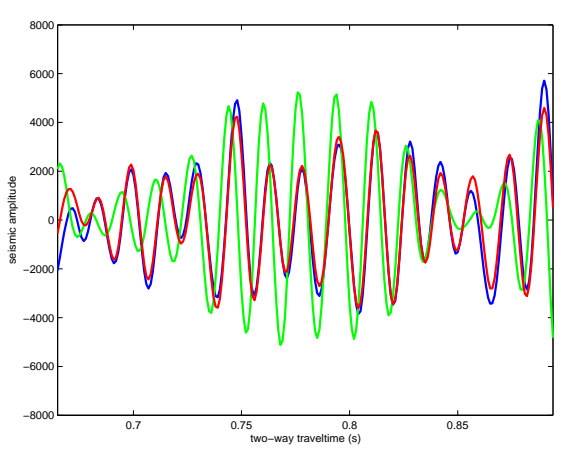

(d) $\mu=1$

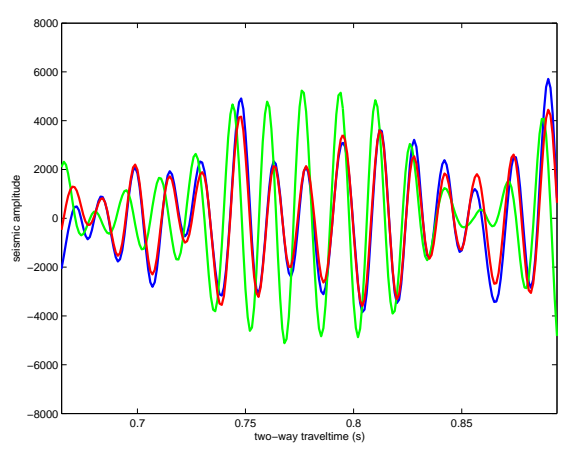

(f) $\mu=2$ 


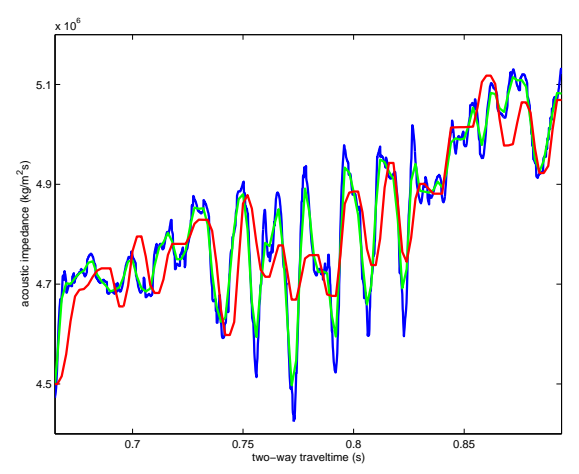

(g) $\mu=4$

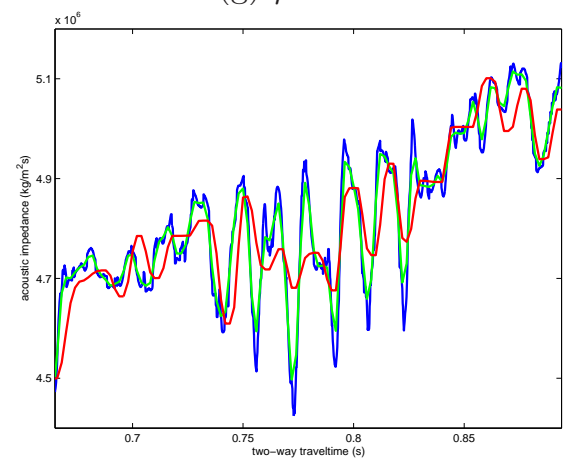

(i) $\mu=8$

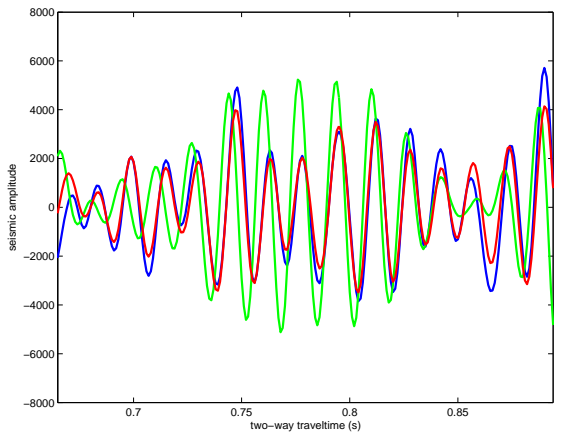

(h) $\mu=4$

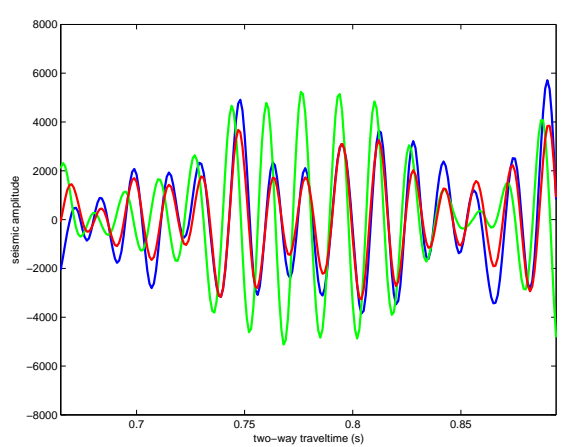

(j) $\mu=8$

Figure 5.4: $\mu$ sensitivity test $(\mu=0.5,1,2,4,8 ; \lambda=1 ; T=2 \mathrm{~ms}$; $M=150)$. Results of 1D inversion of the clinoforms. Left column: the initial model (resampled impedance log, green), the estimated model (red), superimposed on the actual impedance log (blue). Right column: seismic traces of the initial model (resampled impedance log) based synthetic seismic (green), the synthetic seismic (red) and the actual seismic data (blue). 
allowed inside the layers. Moreover, the estimated impedance models tend to exhibit a more distinct layer structure with an increase of the value for $\mu$. An increase of the $\mu$ value limits the number of layers in the estimated models. To be more precise, Figure 5.4(a) $(\mu=0.5)$ shows that two separate thin layers can be distinguished on the estimated impedance model at TWT $0.83 \mathrm{~s}$, while Figure 5.4(i) depicts that those two layers have been merged into a single thicker layer for a high value of $\mu=8$. The reason why this effect is only observed at one location can be easily explained. The properties of these two separate layers show the smallest difference from an acoustic point of view, for the entire model (Figure 5.4(a), TWT 0.83 s). Hence these two layers were the first to be affected by an increasing value for $\mu=8$, i.e., they were forced to be merged. Nevertheless, the same behavior is expected for the remaining layers in the acoustic impedance model but for much higher $\mu$ values.

In addition, the amplitude difference of the entire estimated impedance models decreases for the high $\mu$ values, i.e., the impedance models show a tendency to flatten, compare Figure 5.4(a) and Figure 5.4(i).

The analysis of the seismic section (right column) reveals that the overall mismatch between the actual seismic and the estimated synthetic traces increases with an increase of $\mu$ values, as was expected. The mismatch is mainly seen as a difference in the absolute values of the seismic amplitudes. This holds especially for the models with high $\mu=4$ and $\mu=8$ values (Figure 5.4(h) and Figure 5.4(j)). The output is consistent with the results of the estimated impedance models (left section), that show flattening of the estimated impedance model amplitudes for the mentioned $\mu$ values. In particular, the effect is clearly observed, when tracking the seismic response of the estimated impedance models for the different $\mu$ values of the aforementioned thin layers at TWT $0.83 \mathrm{~s}$. In case of a low $\mu=0.5$, two layers can 
be distinguished (Figure 5.4(b)) and the seismic amplitude mismatch between the actual trace and the estimated synthetic trace is much lower, compared to mismatch for the high $\mu=8$ value (Figure $5.4(\mathrm{j})$ ).

$T$ Sensitivity The regularization parameters $\lambda, \mu$ and the sampling step $T$ are believed to be dependent. Hence, the $T$ sensitivity test is divided into two parts. In the first test, the $\mu$ parameter is kept constant and $\lambda$ is kept fixed in a second test, respectively. These tests are expected to show the behavior of the estimated impedance models and the synthetic seismic traces depending on a finer sampling step $T=1 \mathrm{~ms}$.

Figure 5.5 shows the result of the first test. To depict the difference in behavior of the estimated impedance models and the synthetic seismic traces for the finer sampling $(T=1 \mathrm{~ms})$, the results should be compared to Figure 5.3 ( $T=2 \mathrm{~ms}$ ).

What can be observed is that the estimated impedance models have a somewhat more blocky structure. This outcome can be related to the condition of the TV criterion, that assumes that the trace sampling should be fine enough, i.e., there are many samples inside a single layer. Hence, the more successive samples in the impedance model that have similar acoustic properties, the more a distinct layer is formed. The parameter $\lambda$ enforces the a priori information on those layers. Again, the effect is especially well observed on the thin layers with similar acoustic properties. Unlike the inversion result for the moderate sampling $T=2 \mathrm{~ms}$ (Figure 5.3(a)), in case of the finer sampling $T=1 \mathrm{~ms}$, the above mentioned layer at TWT $0.83 \mathrm{~s}$ is split into two thin layers, even for the lowest $\lambda=0.5$ (Figure $5.5(\mathrm{a})$ ).

In addition, the combination of the finer $T=1 \mathrm{~ms}$ sampling step and high $\lambda=2$ values allows layers that have smaller time thickness to be 
estimated. For example, a thin layer at TWT $0.79 \mathrm{~s}$ was found (Figure 5.5(e)), whereas it remains indicated in Figure 5.3(e) $(T=2 \mathrm{~ms})$.

The amplitudes of the estimated impedance models are less flattened for the finer sampling step $T=1 \mathrm{~ms}$ compared to the same results for $T=2 \mathrm{~ms}$. The estimated amplitudes of the layer at TWT $0.77 \mathrm{~s}$ indicated on Figures 5.5(e) and 5.3(e) is a good example.

As expected, the results of the corresponding synthetic seismic traces for different values of $\lambda$ given in Figure 5.5 reveal that an increase of the sampling rate does not influence this estimation much, see Figure 5.3 for the comparison.

The results of the second $T$ sensitivity test are given in Figure 5.6 and are compared with results for the lower sampling step $T=2 \mathrm{~ms}$ (Figure 5.4). In agreement with the outcome of the first test, the layers on the estimated acoustic impedance models are more distinct for a finer $T=1 \mathrm{~ms}$ sampling step due to the TV criterion.

Analogous to the result of the $\mu$ sensitivity test for $T=2 \mathrm{~ms}$ (Fig-

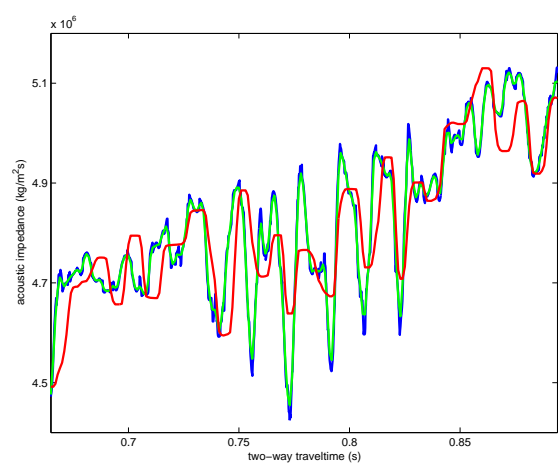

(a) $\lambda=0.5$

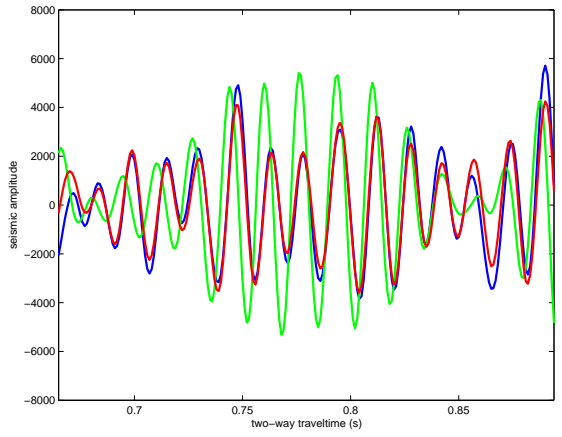

(b) $\lambda=0.5$ 


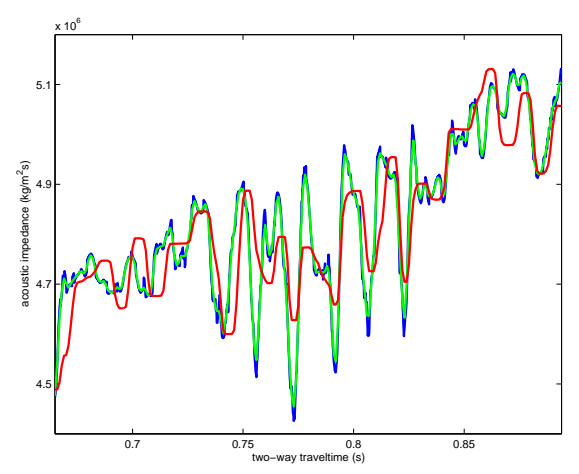

(c) $\lambda=1$

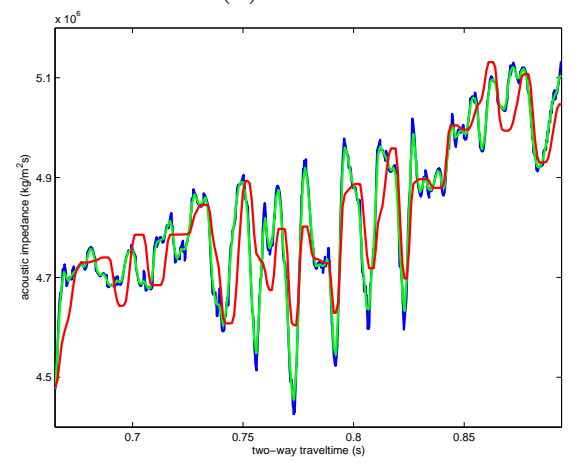

(e) $\lambda=2$

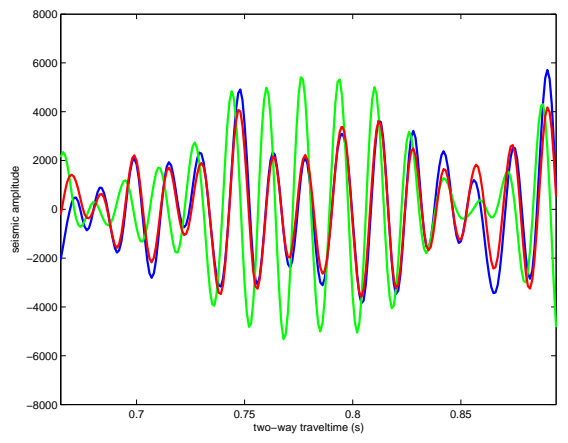

(d) $\lambda=1$

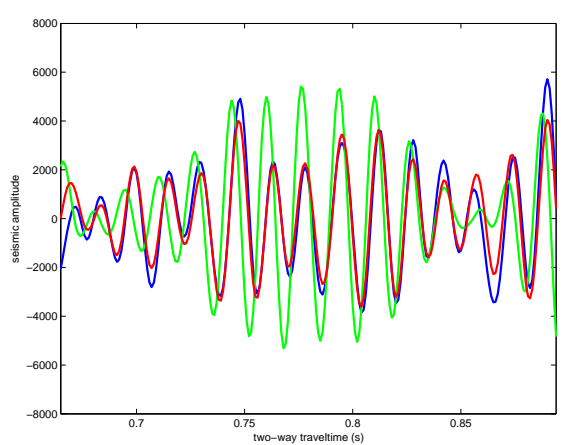

(f) $\lambda=2$

Figure 5.5: Sampling rate $T$ sensitivity test $(\mu=2 ; \lambda=0.5,1,2$; $T=1 \mathrm{~ms} ; M=300)$. Results of $1 \mathrm{D}$ Inversion of the clinoforms. Left column: the initial model (green), the estimated models (red), superimposed on the actual impedance log (blue). Right column: seismic traces of the initial model (resampled impedance log) based synthetic seismic (green), the synthetic seismic (red) and the actual seismic data (blue). 


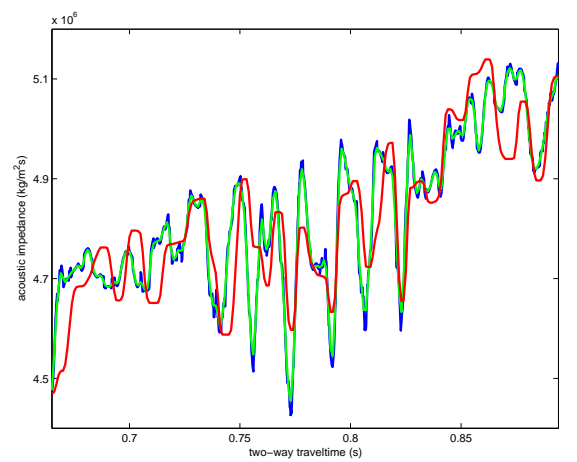

(a) $\mu=0.5$

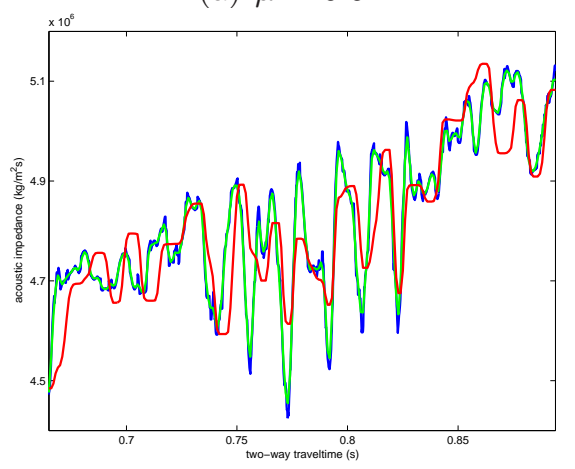

(c) $\mu=1$

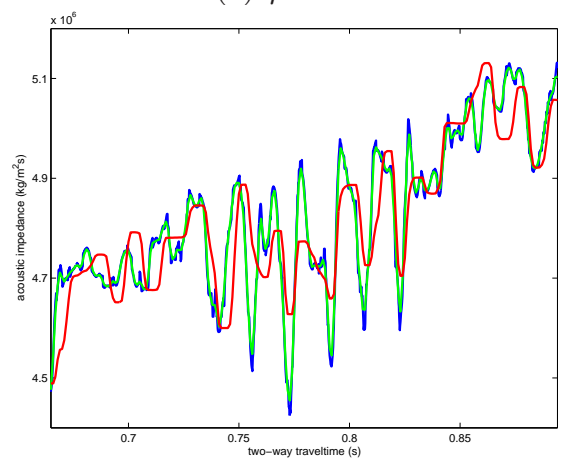

(e) $\mu=2$

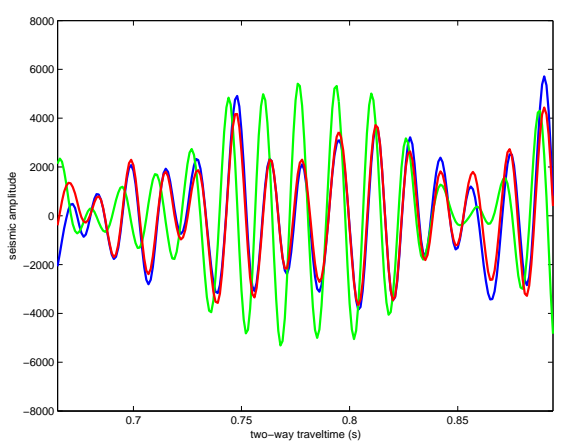

(b) $\mu=0.5$

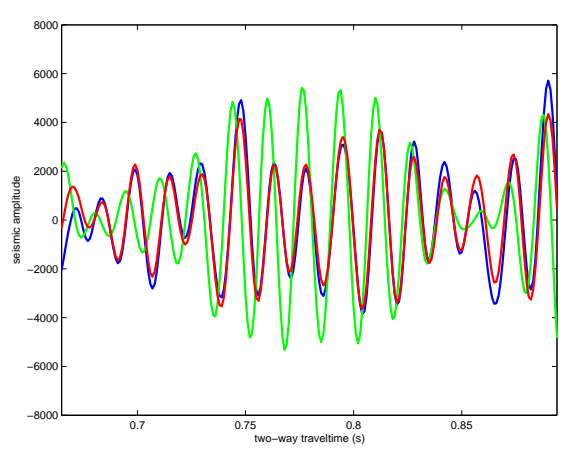

(d) $\mu=1$

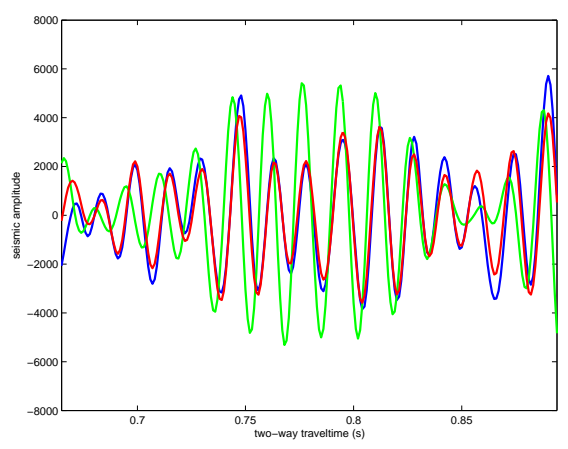

(f) $\mu=2$ 


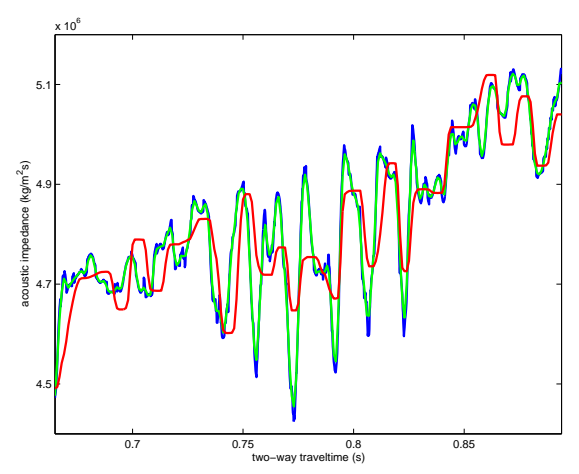

(g) $\mu=4$

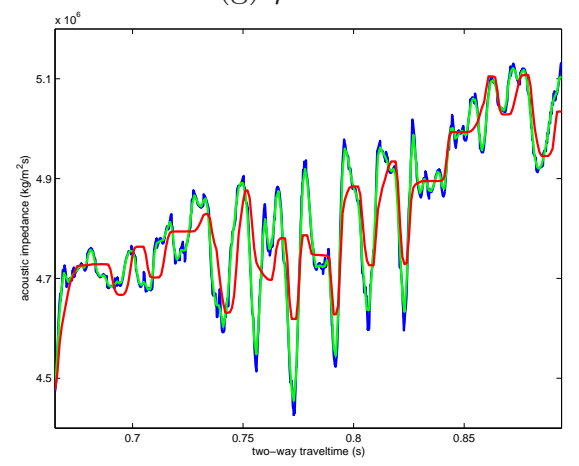

(i) $\mu=8$

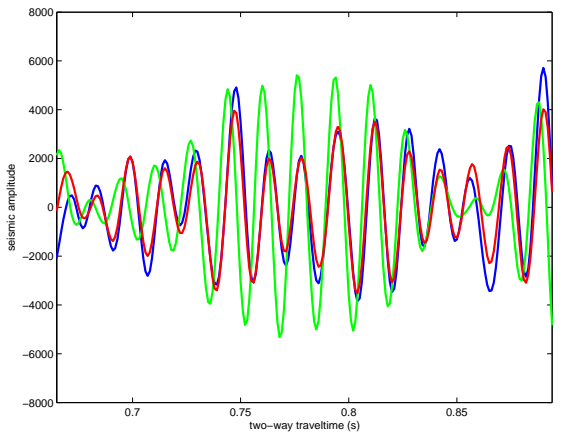

(h) $\mu=4$

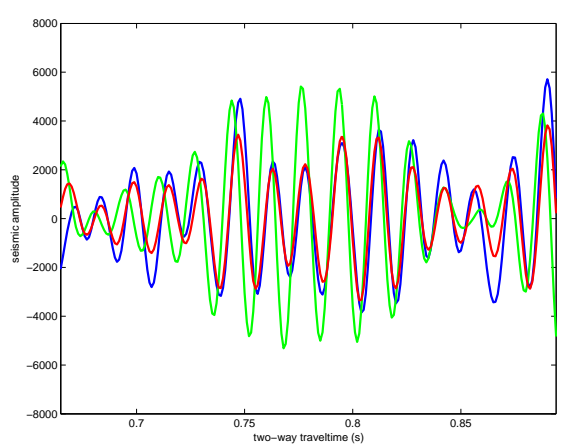

(j) $\mu=8$

Figure 5.6: Sampling rate $T$ sensitivity test $(\lambda=1 ; \mu=0.5,1,2,4,8$; $T=1 \mathrm{~ms} ; M=300)$. Results of 1D inversion of the clinoforms. Left column: the initial model (green), the estimated models (red), superimposed on the actual impedance log (blue). Right column: seismic traces of the initial model (resampled impedance log) based synthetic seismic (green), the synthetic seismic (red) and the actual seismic data (blue). 
ure 5.4) a flattening effect of the amplitudes of the entire estimated impedance models with increasing $\mu$ is observed in Figure 5.6. However, this effect is much weaker for the finer sampling step $T=1 \mathrm{~ms}$. A good example is a layer at TWT $0.84 \mathrm{~s}$ on the estimated impedance model for a high $\mu=8$ parameter and fine sampling $T=1 \mathrm{~ms}$ (Figure 5.6(i)) that is not recognizable anymore on the corresponding plot (Figure 5.4(i)). Another evidence is given by two layers at TWT $0.77 \mathrm{~s}$ and TWT $0.79 \mathrm{~s}$ on the estimated impedance model plotted in Figure 5.4(i). These layers have much lower amplitudes than the ones on the corresponding plot for a high $\mu=8$ and fine sampling $T=1 \mathrm{~ms}$ given in Figure 5.6(i). The reason behind it is as follows: when the number of samples used in the impedance model is getting smaller, then the sensitivity of the model to the small fluctuations in the acoustic properties is reduced. As a consequence the values of the samples are forced to approach the average.

Another interesting feature that can be noted from the result of this test is that for the fine sampling very thin layers can be estimated. Two examples can be selected to illustrate this. The first one holds for the low $\mu=0.5$ regularization. Comparison of Figure 5.4(a) and Figure 5.6(a) depicts that there were a number of thin layers estimated for the finer sampling $T=1 \mathrm{~ms}$ at TWT $0.76 \mathrm{~s}$, TWT $0.81 \mathrm{~s}$, TWT $0.84 \mathrm{~s}$, while those layers can not be distinguished on the corresponding plot for the moderate sampling $T=2 \mathrm{~ms}$. The second example is for the higher $\mu=8$ parameter. Two thin layers at TWT $0.78 \mathrm{~s}$ plotted in Figure 5.6(i) were estimated, whereas on the corresponding plot in Figure 5.4(i) for the moderate sampling they do not show up. We believe that, in case of the finer sampling $T$ of the model, there is enough freedom for the vertical gradient to form an intermediate layer for a group of successive samples, that have slightly different acoustic properties from the surrounding layers. This effect is stronger for the cases when the lower $\mu$ values are applied, since the influence of the 
TV criterion is smaller. This means that the neighboring samples in the model are less forced to group in one layer, even though these samples have similar acoustic properties.

The behavior of the synthetic seismic traces for the finer sampling step $T=1 \mathrm{~ms}$ (Figure 5.6) is similar to the corresponding synthetic seismic traces for a moderate sampling step $T=2 \mathrm{~ms}$ (Figure 5.4). Namely, the mismatch between the actual and the synthetic seismic traces increases with increasing $\mu$ values (while the corresponding estimated impedance models tend to level).

\subsubsection{Results}

The acoustic impedance models obtained with the method described in this chapter have in general a good correlation with the well data for the different $\lambda, \mu$ and $T$ values, within the chosen range of the regularization parameters (Table 5.1). The 1D inversion results yields a good overall match with the actual seismic trace as well. In addition, the vertical resolution of the current method is high, and lies beyond the seismic resolution. The results shows that details (layers) with thicknesses as small as $1 / 10^{\text {th }}$ of the source wavelength are well resolved.

Unlike the previously presented method, more than one regularization parameter is involved in the cost function of the current method. Moreover, those regularization parameters are dependent on each other. Theoretically, the choice for the optimal regularization parameters should be based on the complex analysis of the 1D inversion results together with the pseudo 2D inversion results, similar to the method given in Chapter 4. However, in that case, the number of regularization parameters to be perturbed and investigated in the pseudo $2 \mathrm{D}$ inversion test is very high (90 combinations). In addition, this method 
is computationally expensive since a much larger number of parameters needs to be estimated. Preferably, the possibility to fix one, or, if possible more of the parameters, based on the output of the 1D inversion tests, is highly desired. Therefore, the main goal of these tests was to investigate the level of sensitivity of the inversion procedure to the parameters $\lambda, \mu$ and $T$. Based on the $\lambda$ sensitivity tests it appears that this parameter has the least influence on the $1 \mathrm{D}$ inversion results. Hence, $\lambda$ equal to 1 was picked and will be used in the subsequent pseudo $2 \mathrm{D}$ inversion tests. The results of the $T$ sensitivity tests show that $T$ equal to $1 \mathrm{~ms}$ allows more distinct layering to be formed, i.e., it provides a more accurate estimation of the acoustic impedances than the ones with $T=2 \mathrm{~ms}$. Evidently, therefore, $T=1 \mathrm{~ms}$ should be used in the pseudo $2 \mathrm{D}$ inversion test. The $\mu$ sensitivity tests indicated the general influence of this parameter only. A unique choice of the optimal regularization parameters based on the $1 \mathrm{D}$ inversion tests can not be made, as it is still quite difficult to predict the influence of the parameter on this pseudo $2 \mathrm{D}$ inversion procedure. Consequently, the entire range of $\mu=0.5, \mu=1, \mu=2, \mu=4$ and $\mu=8$ is retained and will be used for the pseudo $2 \mathrm{D}$ inversion tests.

\subsection{Inversion of the Clinoforms}

\subsubsection{Formulation of the Inverse Problem}

The parameter vector to be estimated for every trace consists of the acoustic impedances, sampled with a constant sampling rate $T$ (equation (4.1)):

$$
\boldsymbol{x}^{k}=\left[Z_{1}^{k}, \ldots, Z_{M}^{k}\right]
$$

where $M=300$ is the number of samples per trace, $k=1, \ldots, K$, where $K$ is the number of traces in the sequence to be inverted. Again, 
the seismic section containing $K=70$ traces, situated on the left-hand side of the well was chosen for the test.

Similar to the method described in the previous chapter, the inversion of a seismic section is done trace by trace. However, the inversion technique is not the same for every trace. The inversion procedure is different for the seismic trace closest to the well, in the sense of the cost function and its regularization terms.

Inversion of the Seismic Trace Closest to Well Analogous to the method presented in Chapter 4, the inversion process starts from the trace closest to the well. The inversion procedure for this trace employs the modified cost function $F(\boldsymbol{x})$, given in equation (5.5), and described in the previous section. Like in the $1 \mathrm{D}$ case, the well log data are used as a priori information.

Inversion of the Remaining Seismic Section The inversion procedure for all the remaining seismic traces along the clinoform sequence, starting from the trace that is second closest to the well, employs again an altered modified cost function. In this method, as mentioned in the introduction, we propose to modify the original cost function $F(\boldsymbol{x})$ (equation (2.17)) by including two additional terms. The first additional term was a vertical operator that favors a sparse solution. The second additional term in $F(\boldsymbol{x})$ is a so-called horizontal operator and is explained below (equation (5.7)).

The initial model for the trace second closest to the well is the result (in terms of estimated acoustic impedances) of the first trace closest to the well. In contrast to method presented in Chapter 4, no normalization of the initial model is needed, due to a constant sampling step of the traces. In a similar manner, the resulting estimated parameters of the 
just inverted traces serve as the initial models for all the successive traces.

\subsubsection{Horizontal Operator}

The horizontal operator is a sum of absolute differences between lateral neighboring samples in the current $(k)$ and the previous $(k-1)$ seismic trace. This term accomplishes two tasks at once. First, it promotes continuity of the inversion results along the clinoform sequence. Second, it propagates the a priori knowledge from the well to the current trace. Based on the aforementioned consideration, a hyperbolic instead of the $L_{1}$-norm is used. The original second term in the cost function, which is the weighted $L_{2}$-norm of the deviations of the parameters from their a priori values, can be omitted starting with the second seismic trace from the well. The horizontal operator propagates the well data to the current trace and takes lateral variations of the impedances along the sequence into account at the same time. The functional to be minimized is

$$
\begin{aligned}
F\left(\boldsymbol{x}^{k}\right)=\left(\boldsymbol{y}^{k}-\boldsymbol{g}\left(\boldsymbol{x}^{k}\right)\right)^{T} \boldsymbol{C}_{n}^{-1}\left(\boldsymbol{y}^{k}-\boldsymbol{g}\left(\boldsymbol{x}^{k}\right)\right)+ \\
+\mu \sum_{j}\left(\sqrt{\left(x_{j}^{k}-x_{j-1}^{k}\right)^{2}+1}-1\right)+ \\
+\gamma \sum_{j}\left(\sqrt{\left(x_{j}^{k}-x_{j}^{k-1}\right)^{2}+1}-1\right),
\end{aligned}
$$

where $k>1$ is a trace number. Similar to the vertical operator case (equation (5.5)), the last term in equation (5.7) is also normalized by a factor $\sigma_{\boldsymbol{x}_{i}}^{2}$ (to keep the correspondence with equation (4.4)). 


\subsubsection{Regularization}

The cost function $F(\boldsymbol{x})$ given in equation (5.7) includes two regularization parameters: $\mu$ and $\gamma$. The first one, $\mu$, in front of the vertical operator term, restricts the number of layers in the inverted trace and penalizes fluctuations inside each layer. The second one, $\gamma$, in front of the horizontal operator term, enforces lateral continuity of the impedances. In other words, it controls variations allowed from trace to trace.

The aim of this section is to investigate how to select the appropriate values for $\mu$ and $\gamma$. Due to the absence of a technique that could provide an indication of the optimal values for these parameters, the choice is made empirical, i.e., similar to the previous cases (see Chapter 4$)$.

The difference between the pseudo 2D inversion tests performed in the method described in the previous chapter (they were focused on finding an optimal $\lambda$ regularization) and the current method, is that there are now two weighting coefficients in the cost function $F(\boldsymbol{x})$ involved (equation (5.7)). In addition, the physical significance of these weighting coefficients is different. Hence, a sensitivity tests of the parameters are needed. Taking into account that weighting coefficients $\mu$ and $\gamma$ are dependent, sensitivity tests cannot be performed separately for each one (like in the method described in Chapter 4).

Based on the 1D inversion sensitivity tests the entire range of $\mu$ values was selected for the pseudo $2 \mathrm{D}$ tests, i.e., $\mu=0.5-8$. We presume that an optimal restriction of the number of layers in the acoustic impedance model is within this range.

Taking into account that the horizontal operator is also normalized 
by a factor $\sigma_{\boldsymbol{x}_{i}}^{2}$ (equation (5.7)) the following range of $\gamma$ values was selected: $\gamma=0.5, \gamma=1, \gamma=2$. These values are expected to exhibit the low and the relatively high influence of the horizontal operator term.

The weighting coefficient $\lambda$ is used in inversion of the first trace, closest to the well, only (equation (5.5)), but it still plays an important role in transferring the well data information into the entire sequence. Based on the output of the $1 \mathrm{D}$ sensitivity test, $\lambda=1$ is used in all pseudo 2D inversion tests. Another parameter that was fixed, based on the 1D inversion analysis, is the sampling step $T=1 \mathrm{~ms}$ (equation (4.1)) and the corresponding number of samples $M=300$ (number of parameters to be estimated, equation (5.4)).

The values of all parameters for the pseudo $2 \mathrm{D}$ sensitivity tests are summarized in Table 5.2.

\begin{tabular}{|c|c|c|c|c|c|c|}
\hline$\lambda$ & $\gamma$ & \multicolumn{5}{|c|}{$\mu$} \\
\hline \hline & 0.5 & 0.5 & 1 & 2 & 4 & 8 \\
1 & 1 & 0.5 & 1 & 2 & 4 & 8 \\
& 2 & 0.5 & 1 & 2 & 4 & 8 \\
\hline
\end{tabular}

Table 5.2: Parameters in the pseudo 2D sensitivity tests, sampling rate $T=1 \mathrm{~ms}$

Sensitivity: $\mu$ and $\gamma$ Since there are many parameter combinations to be investigated, the analysis of the sensitivity test will be performed in the following order: first, the extreme cases are considered, second, a group of the potential optimal combinations is selected and investigated closer. 


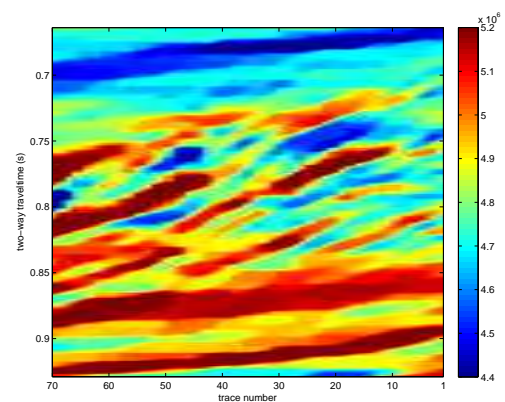

(a) $\gamma=0.5 ; \mu=0.5$

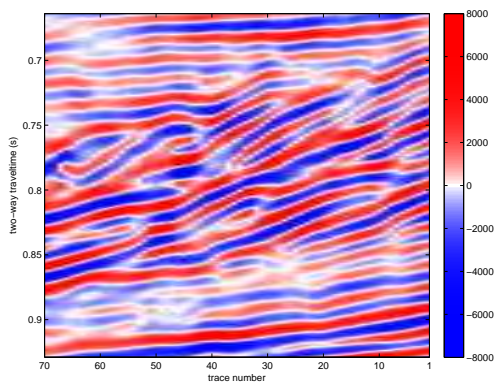

(b) $\gamma=0.5 ; \mu=0.5$

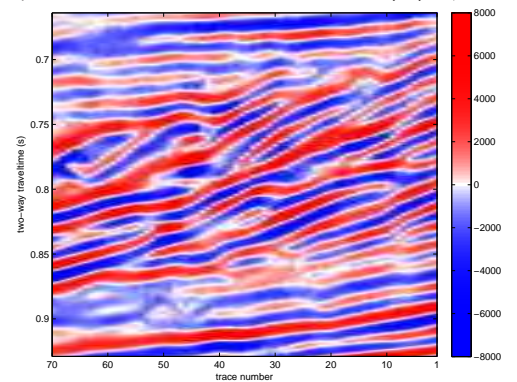

(c) real seismic

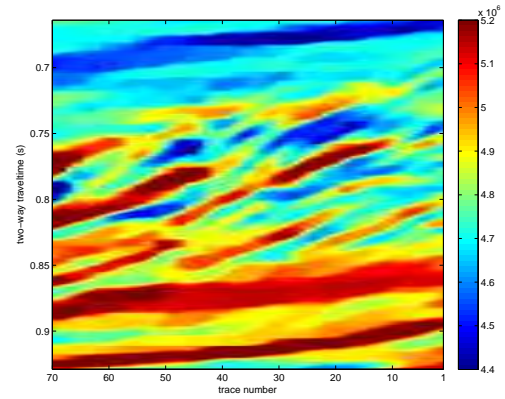

(d) $\gamma=0.5 ; \mu=1$

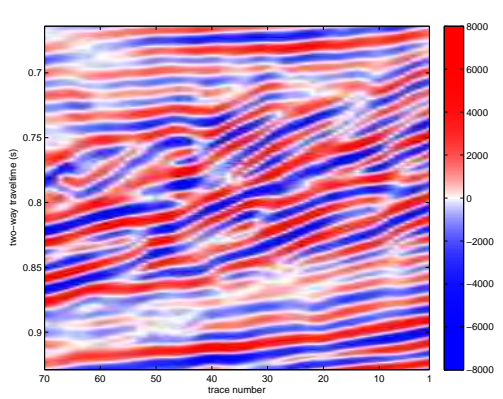

(e) $\gamma=0.5 ; \mu=1$

Figure 5.7: Pseudo 2D $\mu$ and $\gamma$ sensitivity test $(\mu=0.5,1 ; \gamma=0.5)$. Results of the inversion of the clinoforms: left column - the estimated acoustic impedance models, right column - the synthetic seismic, center column - the actual seismic. 
Figure 5.7 illustrates the pseudo 2D inversion results for the combination of the very low values of the parameters $\mu=0.5$ and $\gamma=0.5$ (upper row). As can be observed on the estimated impedance model (left), the layer distinction and the continuity are at a low level. The estimated impedance model for the combination of the next value of the parameter $\mu=1$ at the same low value of the parameter $\gamma=0.5$ is shown in the lower row in Figure 5.7. A similar output is observed, namely a low layer distinction, but to a lesser extent.

The corresponding seismic sections (right column) have in general a good correlation with the real seismic sections but still minor mismatches can be detected.

The result of the pseudo 2D inversion for a combination of the low value of the parameter $\mu=0.5$ but a high value of the parameter $\gamma=2$, is shown in the upper row in Figure 5.8. It appears that the continuity term plays a dominant role in this combination, i.e., artificial horizontal layering is created. The dominance of the lateral continuity term is still obvious, but to a smaller extent than when a slightly higher value of the parameter $\mu=1$ and the same high value of the parameter $\gamma=2$ were used (the lower row in Figure 5.8). However, the layering characteristics of the estimated impedance model is improved.

The effect of the very high lateral continuity prevalence can also be observed in the corresponding seismic sections (the right column).

The number of the identified layers in the estimated acoustic impedance as a function of the seismic trace along the sequence, for combinations of the regularization parameters $(\mu=0.5, \gamma=0.5 ; \mu=1, \gamma=0.5$; $\mu=0.5, \gamma=2 ; \mu=1, \gamma=2)$ is shown in Figure 5.9. As can be observed, the mean number of layers identified (dashed line) fluctuates. The rapid increase in the average number of layers towards a value of 44 occurs when a low parameter $\mu=0.5$ and high parameter $\gamma=2$ 


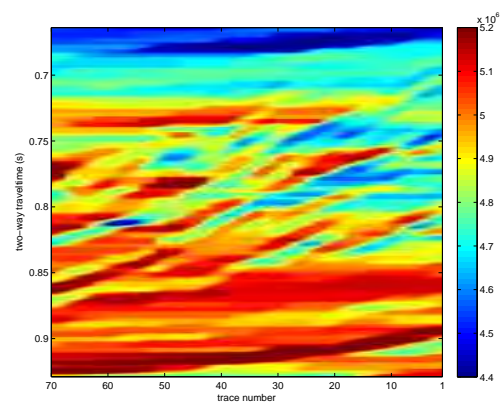

(a) $\gamma=2 ; \mu=0.5$

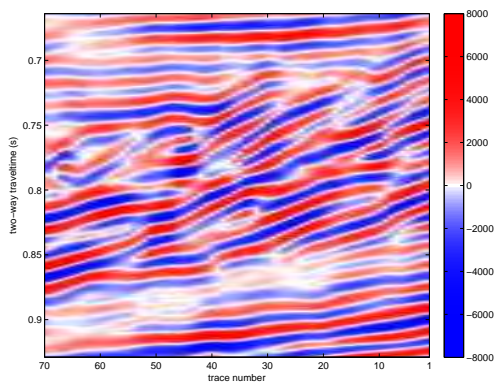

(b) $\gamma=2 ; \mu=0.5$

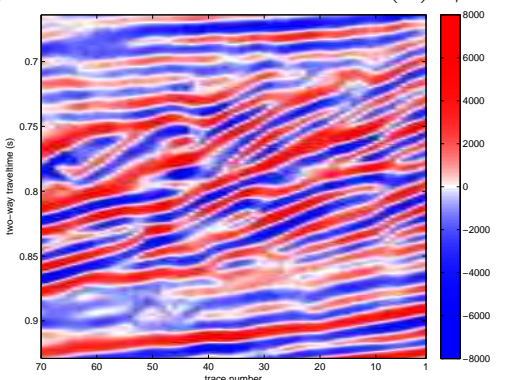

(c) real seismic

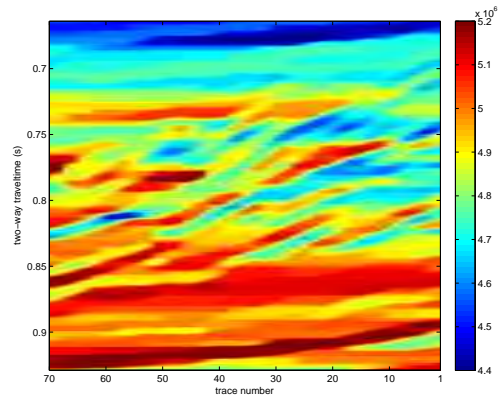

(d) $\gamma=2 ; \mu=1$

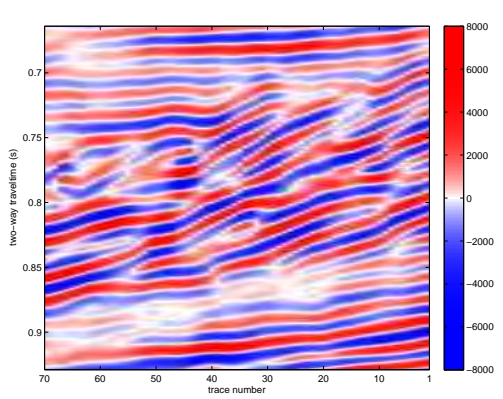

(e) $\gamma=2 ; \mu=1$

Figure 5.8: Pseudo 2D $\mu$ and $\gamma$ sensitivity test $(\mu=0.5,1 ; \gamma=2)$. Results of inversion of the Clinoforms: left column - the estimated acoustic impedance models, right column - the synthetic seismic, center column - the actual seismic. 


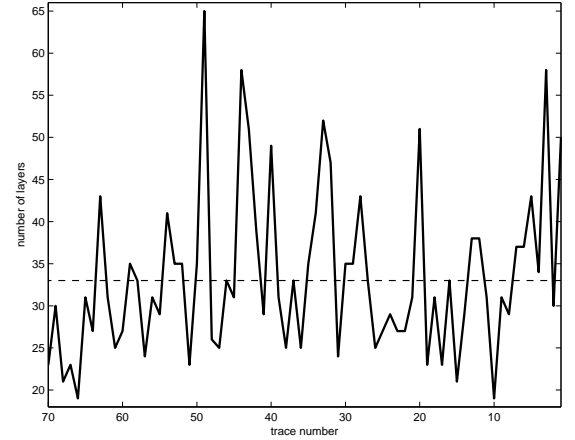

(a) $\gamma=0.5 ; \mu=0.5$

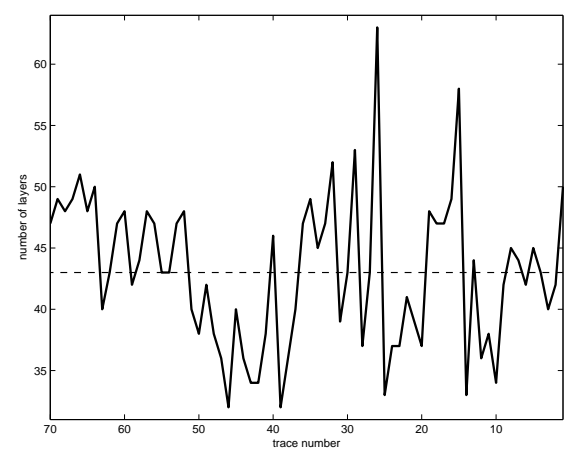

(c) $\gamma=2 ; \mu=0.5$

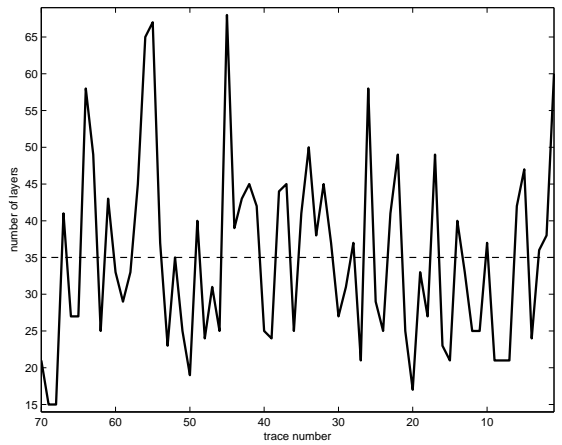

(b) $\gamma=0.5 ; \mu=1$

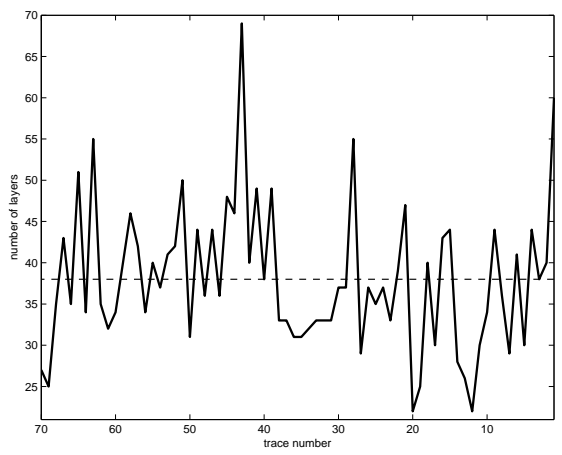

(d) $\gamma=2 ; \mu=1$

Figure 5.9: The number of identified layers in the target zone vs trace number $(\gamma=0.5 ; \mu=0.5,1)$. 
(Figure 5.9(c)) are applied. The average number of layers for other combinations fluctuate around 35. This output correlates with the previous conclusions, i.e., when the influence of the vertical operator is relatively low compared to the one of the horizontal operator, a form of artificial layering is created.

Once the combinations of regularization parameters $\mu$ and $\gamma$ that cause artificial effects were discarded, the group of potential optimal combinations was investigated more thoroughly. The pseudo 2D inversion results are organized in a three-by-three matrix, with parameter $\mu(2$, $4,8)$ increasing from left to right and parameter $\gamma(0.5,1,2)$ increasing from top to bottom. In the lateral direction the amount of layers is restricted and in the vertical direction continuity is promoted.

The estimated impedance models for these combinations of the parameters and the corresponding synthetic seismic sections are displayed in Figure 5.10 and Figure 5.11. Figure 5.12 presents the number of the identified layers for those combinations of the regularization parameters.

There are two attributes that have to be investigated. The first one is the layering and associated with it, the level of detail (vertical direction) of the resulting impedance models. The second attribute is the continuity of the estimated impedance models. The analysis of the corresponding seismic sections (Figure 5.11), and their comparison with the actual seismic is very important since it could assist in the validation process.

Layering. As expected, the general trend for all combinations shows that with an increase of the $\mu$ value, from $\mu=2$ (first column) to $\mu=4$ (second column) the layering gets more distinct. The following increase of the $\mu$ value, from $\mu=4$ (second column) to $\mu=8$ (third column), causes a flatness effect of the impedance models, i.e., the 


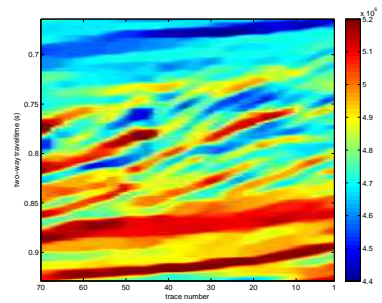

(a) $\gamma=0.5 ; \mu=2$

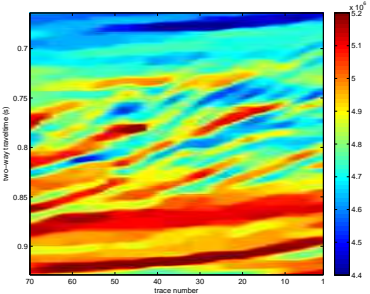

(d) $\gamma=1 ; \mu=2$

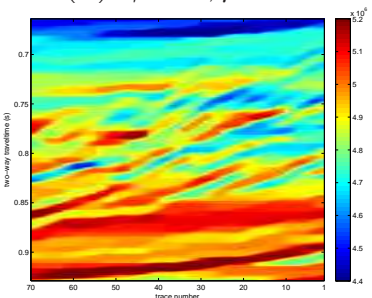

(g) $\gamma=2 ; \mu=2$

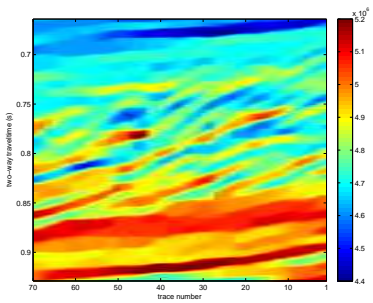

(b) $\gamma=0.5 ; \mu=4$

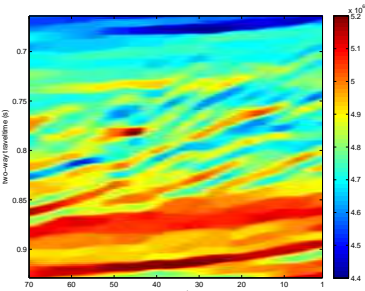

(e) $\gamma=1 ; \mu=4$

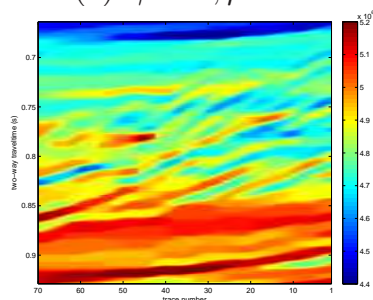

(h) $\gamma=2 ; \mu=4$

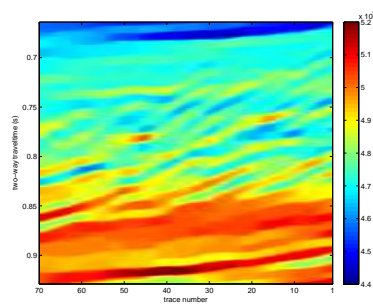

(c) $\gamma=0.5 ; \mu=8$

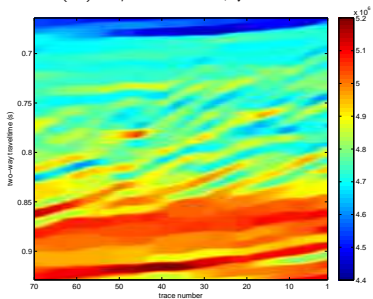

(f) $\gamma=1 ; \mu=8$

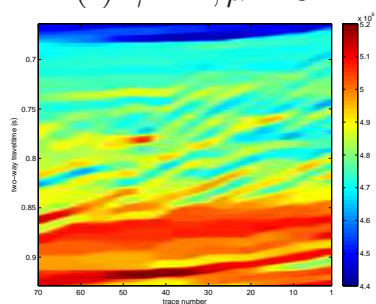

(i) $\gamma=2 ; \mu=8$

Figure 5.10: Pseudo 2D $3 \times 3$ sensitivity test, the estimated acoustic impedance section $(\lambda=1 ; \mu=2,4,8 ; \gamma=0.5,1,2)$. 


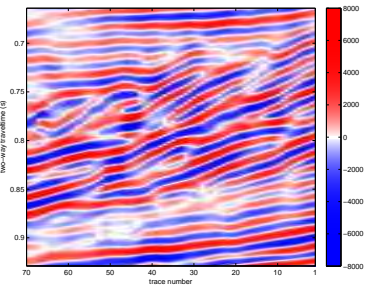

(a) $\gamma=0.5 ; \mu=2$

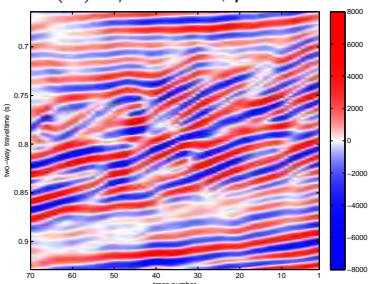

(d) $\gamma=1 ; \mu=2$

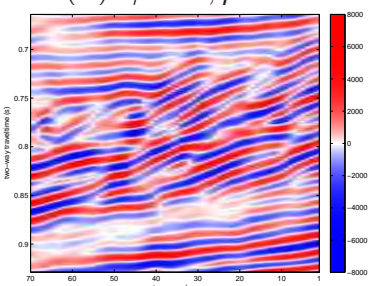

(g) $\gamma=2 ; \mu=2$

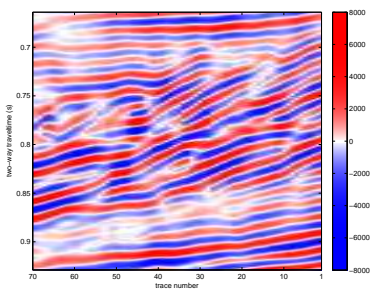

(b) $\gamma=0.5 ; \mu=4$

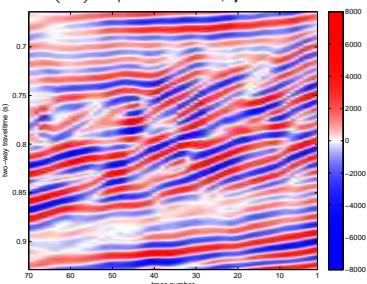

(e) $\gamma=1 ; \mu=4$

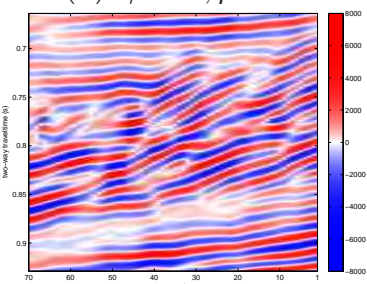

(h) $\gamma=2 ; \mu=4$

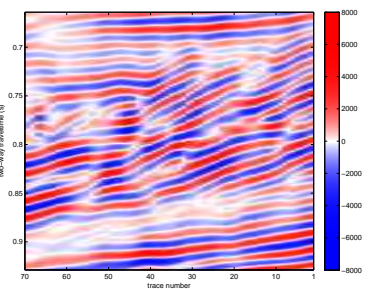

(c) $\gamma=0.5 ; \mu=8$

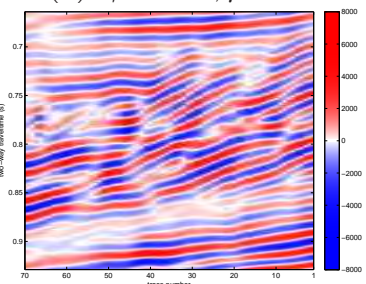

(f) $\gamma=1 ; \mu=8$

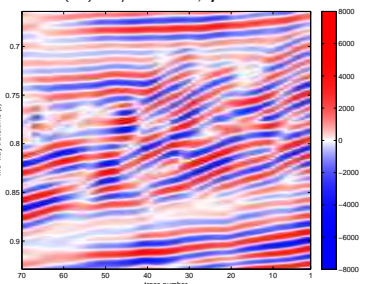

(i) $\gamma=2 ; \mu=8$

Figure 5.11: Pseudo 2D $3 \times 3$ sensitivity test, synthetic seismic section $(\lambda=1 ; \mu=2,4,8 ; \gamma=0.5,1,2)$. 

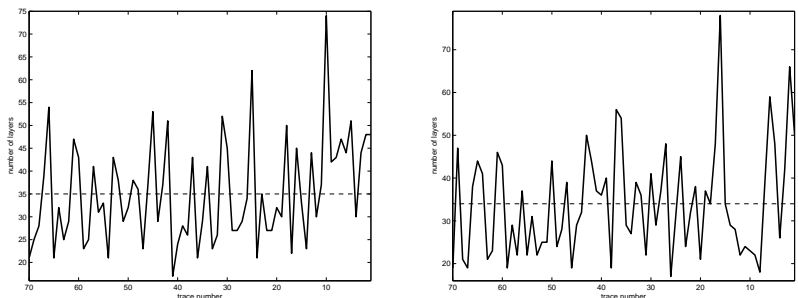

(a) $\gamma=0.5 ; \mu=2$

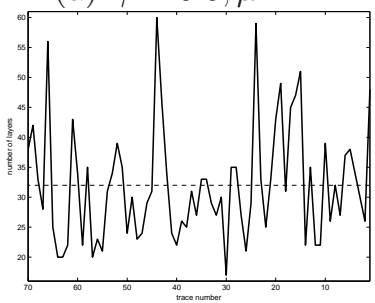

(d) $\gamma=1 ; \mu=2$

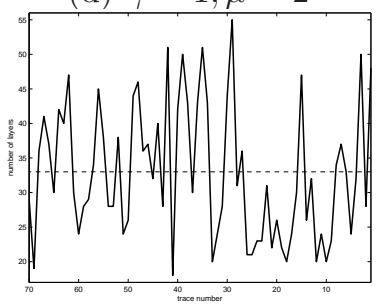

(g) $\gamma=2 ; \mu=2$

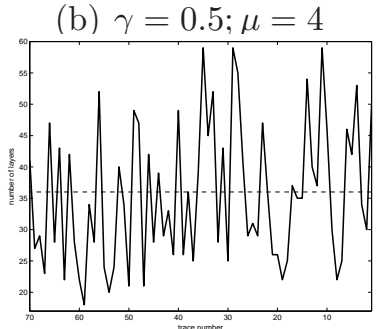

(e) $\gamma=1 ; \mu=4$

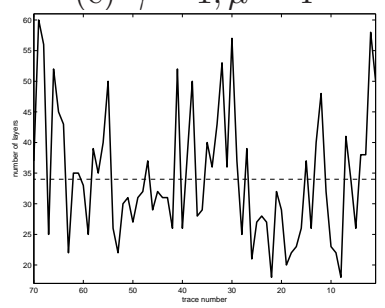

(h) $\gamma=2 ; \mu=4$

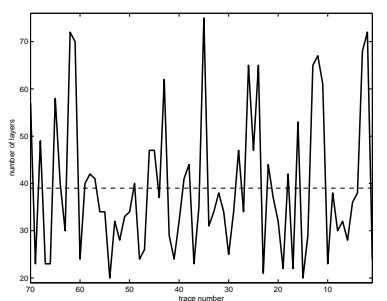

(c) $\gamma=0.5 ; \mu=8$

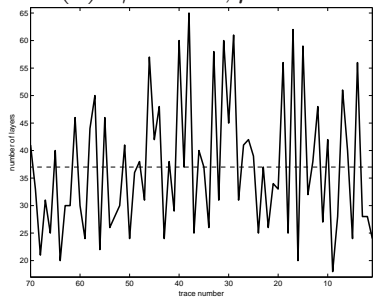

(f) $\gamma=1 ; \mu=8$

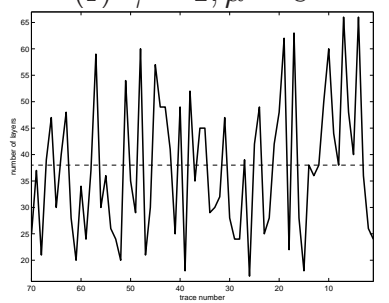

(i) $\gamma=2 ; \mu=8$

Figure 5.12: The number of identified layers in the target zone vs trace $\operatorname{number}(\gamma=0.5,1,2 ; \mu=2,4,8)$. 
vertical operator levels the estimated impedances to an average. In that way, layers that are relatively thin are being grouped in a single, thick layer. This effect was also detected on the 1D $\mu$ sensitivity test.

The analysis of the corresponding seismic sections (Figure 5.11) shows that if $\mu$ increases from $\mu=2$ (first column) to $\mu=8$ (third column), then the seismic phases that correspond to different reflection times (depth) tend to have equal time thicknesses. The effect is especially well observed for combinations with a low value of the parameter $\gamma=0.5$ (first row).

Continuity. This attribute can be described by means of two criteria, the intensity of the amplitudes of the estimated acoustic impedance models, and their structures.

As expected, the general trend for all combinations is that the contrast of the acoustic impedance models decreases when the $\gamma$ value changes from $\gamma=0.5$ (first row) to $\gamma=2$ (third row). This trend is especially visible for the combinations with a low parameter $\mu=0.5$ (left column) and almost negligible for the combinations with a high parameter $\mu=8$ (third column).

Structure-wise, the tendency is that the thicknesses of the particular layers fluctuate less and exhibit better stability along the sequence with an increase of the $\gamma$ value from $\gamma=0.5$ to $\gamma=2$ (first and third rows). Again the effect is better observed for the low value of the parameter $\mu=0.5$ (first column) and it decreases for the higher $\mu$ values.

The two aforementioned attributes are less evident in the synthetic seismic sections (Figure 5.11), but are still recognizable. The amplitudes of the seismic sections overall decreases when $\gamma$ values rise from $\gamma=0.5$ (first row) to $\gamma=2$ (third row). Similar to the estimated 
acoustic impedance sections, the effect is better seen for low $\mu=0.5$ values (first column) and almost disappears for the high $\mu=8$ values (third column).

The augmented layering with the increase of the parameter $\gamma$ from $\gamma=0.5$ to $\gamma=2$ is very well observed for the combination with a low value of the parameter $\mu=0.5$ (first column). In particular, tracing the lower left corner of the seismic images belonging to the first column from the top to the bottom, it is evident that the seismic phase at TWT $0.9 \mathrm{~s}$ almost disappears on Figure $5.7(\mathrm{~b})$, whereas it is forcedly prolonged for the higher $\gamma=2$ value (Figure $5.11(\mathrm{~g})$ ). Increasing the parameter $\gamma$ from $\gamma=0.5$ to $\gamma=2$ in combination with a high value of the parameter $\mu=8$ (third column) reveals another effect: the entire layer structure of the synthetic seismic image becomes exaggeratedly horizontal (Figure 5.11(i)). In addition, the thicknesses of the seismic phases that correspond to different reflection times are constant along the sequence.

The number of identified layers for the above mentioned combinations, given in Figure 5.12, shows that the average fluctuates around 35, but for the given combinations the span is not substantial.

\subsubsection{Results}

Since there is no objective way to determine the optimum values of the regularization parameters, a subjective approach was applied in the approach presented here. Due to the fact that the number of the regularization parameters to be selected is quite high, the approach is divided into three steps.

First, a 1D sensitivity test of the regularization parameters, $\lambda$, in front of the a priori misfit term, $\mu$, in front of the vertical operator term 
given in equation (5.5), and the sampling step $T$ (equation (5.4)) was applied. This test allowed us to select the optimum parameter $\lambda$ equal to 1 , and the optimal parameter $T$ equal to $1 \mathrm{~ms}$. Those values were used in the pseudo 2D test.

Second, a pseudo 2D sensitivity test of the weighting coefficients in front of the vertical and horizontal operators was performed. The test permits us to select the group of the potential optimal combinations of the regularization parameters. Both the 1D and pseudo 2D sensitivity test helps to understand the dependence on the parameters and thereby assisted in guiding the search for the optimum values.

The last step is the expertise of a geologist who can choose the most realistic solution from a geological perspective, from the selected group of the potential optimal regularization combinations.

Based on this approach, the following combination of the regularization parameters was chosen as optimal: $T=1 \mathrm{~ms}, \lambda=1, \mu=4, \gamma=1$. Figure 5.13 shows the estimated impedance model, the corresponding synthetic seismic section for the optimal regularization parameters, together with the actual seismic and the number of the identified layers.

The choice was made based on several criteria: a good match of the synthetic seismic section and the actual seismic section, and a realistic geological interpretation of the resulting 2D impedance model, based on the resulting vertical resolution and its lateral continuity.

The corresponding synthetic seismic section (Figure 5.13(b)) reveals a good match with the field data (Figure 5.13(d)), in the sense that the seismic amplitude values and the seismic phase thicknesses show a good correlation between the sections.

From a geological point of view, the chosen optimal regularization com- 


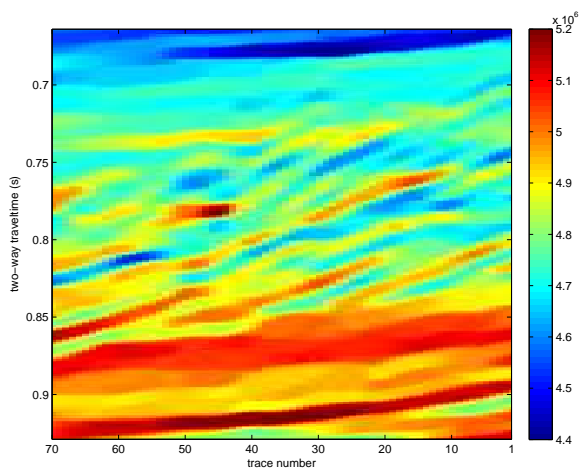

(a) estimated acoustic impedance model

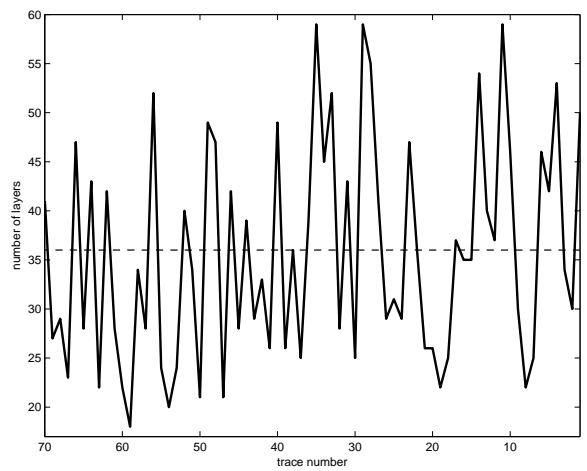

(c) number of the identified layers

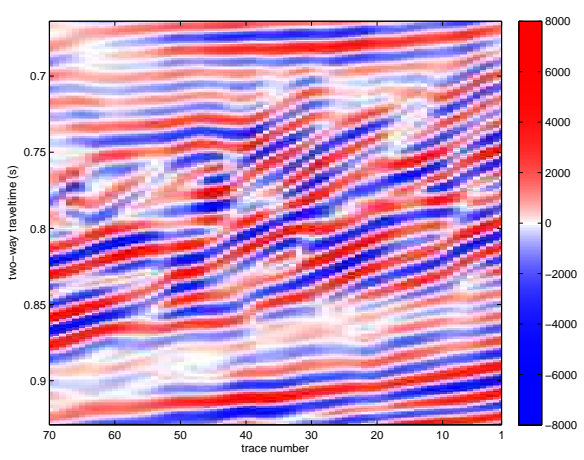

(b) synthetic seismic

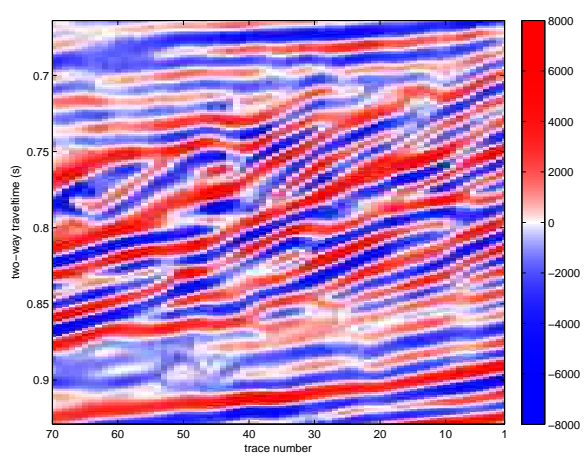

(d) actual seismic

Figure 5.13: Pseudo 2D seismic inversion results with the optimal regularization applied: $T=1 \mathrm{~ms}, \lambda=1, \mu=4, \gamma=1$.

bination provides the acoustic impedance model with realistic properties. The layers gently overlapping each other follow the sigmoidal shapes. The properties of the layers do not differ much from the well data, which are regarded as ground truth. At the same time, given the length of the chosen seismic section to be inverted (70 traces, with 
$25 \mathrm{~m}$ steps in between), lateral changes occur, both in the impedance values and in the thickness of the layers. The layers realistically appear and disappear in accordance with what can be expected in a clinoform sequence.

We observe that the chosen impedance model is characterized with a high level of details that can be observed from the resulting image. A good example to demonstrate this are three consecutive, relatively thin layers at TWT $0.75 \mathrm{~s}$, trace numbers from 1 to 35 , that are well observable on the acoustic impedance model (Figure 5.13(a)).

We suppose that the continuity of the chosen impedance model is close to optimum. It propagates the well data along the sequence, but at the same time it allows changes in the layers properties in the lateral direction. Overall, the amplitude intensity of the entire model corresponds to the well data, meaning that acoustic impedance values do not change drastically along the sequence. At the same time, some reasonable fluctuations are detected. The same applies to the thicknesses of the estimated layers, i.e., in general they remain in accordance with the well data, but are able to change along the profile within a sensible range. The optimum combination of the regularization parameters used in this estimated acoustic model, does neither create artificially continuous layering nor false, horizontally leveled structures.

The best pseudo 2D inversion result (when the optimal combination of the regularization parameters is applied) is situated right in the middle of the selected group (three-by-three matrix) of parameters. In general, all pseudo 2D results do not differ drastically from each other. However, moving from the selected middle cell (the acoustic model with the optimal regularization applied) to any other result within the three by three matrix of the pseudo 2D results (Figure 5.10), reveals that those results fail to satisfy one or more of the selection criteria. 
For example, moving towards high $\mu=8$ values (third column) shows the insufficient level of detail in the resulting model, i.e., the thin layers are merged with surrounding layers. A shift towards the low $\mu=0.5$ values (first column) fails to comply with the geological criterion, i.e., the impedance values change unrealistically much along the clinoform sequence. Moving towards the higher $\gamma=2$ values (third row) fails to satisfy both the geological criterion, i.e., the impedance models have artificial horizontal layering, and the match with the actual seismic deteriorates, i.e., the seismic amplitudes and phases' thicknesses have artificial constant values. A shift in the direction of the low $\gamma=0.5$ values (first row) results in problems to fulfill either the sufficient level of detail criterion or the geological realism criterion, depending on the $\mu$ values, i.e., layers either have unrealistically big changes in the impedance values along the sequence or are suppressed too much.

In view of these facts, we believe that our choice of the optimum combination of the regularization parameters is reasonable and acceptable, although we have to mention that it still contains a certain degree of subjectivity. 


\section{Chapter 6}

\section{Discussion, conclusions, and recommendations}

The present study has resulted in two inversion methods which allow characterization of a clinoform sequence at the subseismic scale. The methods are interdisciplinary, and quantitative. The methods are especially considered to be useful when seismic data alone do not reveal the actual detailed reservoir architecture, which can be the case either because of their low vertical resolution or exceedingly thin layering.

The proposed inversion methods are not fully $2 \mathrm{D}$, but are more than a series of independently processed 1D inversions. Although it is trivial to rewrite it as true 2D inversion, we choose to implement it as a 'recursive' series of 1D inversions to substantially reduce the computational costs. To stress the enforced continuity along the geological structure, we call it pseudo $2 \mathrm{D}$ inversion.

The choice for a deterministic inversion technique was equally motivated by two aims. The first one is the possibility of including structural information - in this case the clinoform shapes - into the inver- 
sion process. The second one is the possibility to incorporate the well information into the technique and thereby to increase the vertical resolution of the inversion results and to integrate different sources of information into a common earthmodel.

The integration of seismic data, well data and geological knowledge of the reservoir architecture allowed us to construct high-resolution, 2D impedance models of the subsurface.

Both seismic inversion methods presented here show encouraging results and show a good match with the measured seismic data and with the well information. The methods are consistent with the initial models (well-log), are constrained by it and yet are flexible enough to detect deviations from the models which are dictated by the seismic data and due to changes in the geological settings of the reservoir.

A benefit of both methods is that the resulting acoustic impedance models are layer models rather than reflection boundaries. We chose acoustic impedance as a characteristic property because it can be linked to reservoir properties such as porosity and, in some instances, fluid saturation. Moreover, acoustic impedance models can be interpreted by geophysicists, petrophysicists, geologists and petroleum engineers.

The inverted impedance models exhibit a vertical resolution between the resolution of the well logs and the vertical resolution of the seismic data. Compared to the seismic data, the increase of vertical resolution improves the interpretation of the internal architecture of the clinoform. 


\subsection{Stratigraphic model-based, low-param- etrization seismic inversion}

The novelty in this method is the integration of the lateral highresolution seismic data with the vertical high-resolution well data and a stratigraphic geological model, into the framework of a deterministic inversion method, using only a small amount of parameters. The geological models are constructed from quantitative knowledge of the reservoir architecture. This multi-disciplinary approach pays special attention to a proper parameterization of the 2D geological objects. These geological objects guide the inversion, reducing the solution space and increasing the confidence in the final reservoir model.

Since the addressed inverse problem was ill-posed, regularization played an important role in this method. First, a 'L-curve' technique was used on the seismic data and the a priori misfit to analyze the $1 \mathrm{D}$ results. This technique helped to constrain the range of possible values for the trade-off parameters. Then, more subjectively, pseudo $2 \mathrm{D}$ results were analyzed by a geological expert, choosing the best model, corresponding to a specific trade-off parameter. In this way, we did not only attempt to generate accurate results, but also made the method more geologically driven.

The presented seismic inversion method, integrated with stratigraphic geological modeling, showed encouraging results when it was applied to a clinoform sequence from the North Sea. The acoustic properties of the internal layers of the fluvio-deltaic sequence were estimated at a subseismic scale, with layer thicknesses as thin as $1 / 9^{\text {th }}$ of the seismic wavelength. From the estimated acoustic parameters and the layer thicknesses, a 2D depositional model was constructed that demonstrated a high level of detail. 


\section{Advantages}

- The method is capable to parameterize clinoform systems and describe their geometry using a very small number of parameters.

- The estimated sigmoid models provide sufficient extrapolation of the well data along the clinoform sequence and assist in the characterization of the model.

- The estimated sigmoid models are used to steer the 1D trace inversion along the clinoform sequence.

- The method allows lateral changes of impedance properties of the layers along the clinoforms, which typically occurs when going from topset to foreset to bottomset of the clinoforms.

- There are parts of the clinoform that can not be tracked on the seismic image due to the low vertical resolution of the data or convergence of the layers. This effect is especially prominent on the topset and the bottomset parts of the clinoform sequence. The method is able to extrapolate the well information to the disconnected parts of the clinoforms, as well as to the events at the subseismic scale.

- The method offers to be a predictive tool for further occurrences of similar clinoform objects in the sequence. The estimated sigmoid parameters can be used to predict the geometry of a clinoform layer with incomplete information.

- The estimated sigmoid parameters are a useful tool to characterize the changes in clinoform thickness, internal geometry, and style of superposition of multiple clinoforms, that in turn can potentially provide information regarding short-term fluctuations of sediment supply, long-term margin subsidence, and sea level changes. 
- As a result of the parametrization using a small number of parameters, the computational cost of the method is very low.

\section{Limitations}

- In the present study, only one specific environment has been investigated, i.e., a clinoform sequence in a presumably fluviodeltaic setting. This limits the wider application of the method, but a similar approach could be applied to characterize other systems, e.g., channelized sequences.

- The methodology to parameterize objects is more or less limited to the sedimentary environment of the data set, but it is scale-, and - to some extent - shape-independent.

- For this method, it is compulsory that the clinoform structures are at least partially present at the seismic scale.

- Upscaling (smoothing) method of the well data performed in Chapter 3 (Section 3.6.2), could be a potential source of error when finely layered media with detail far below the seismic resolution are studied (simple filtering and re-sampling change the gross behavior of the finely detailed medium). For correct upscaling of detailed log information, effective medium theory should be applied (this topic was beyond the scope of this project). The industry standard method is Backus averaging of moduli. For more information, see Lindsay and van Koughnet (2001).

- The wavelet estimation was done with a zero phase assumption, for simplicity. The wavelet was later checked by visual comparison of the synthetic (the well data (reflectivity) convolved with the estimated wavelet) and the real, close to the well, seismic traces. The good correlation between these two traces was explained by the assumption that the real data set could have been 
filtered with the zero-phase filter during the processing stage. In practice, the assumption of a zero phase wavelet is not always valid and the wavelet (including the wavelet scaling) needs to be thoroughly checked by tying the wells to the seismic. This is especially the case when finely layered media are studied.

- The method includes the lithological interpretation of the well log used to create a 1D initial model of the reservoir. As a consequence, a user-introduced bias is unavoidable.

- In the current method, while constructing the 1D initial model, the number of layers composing the clinoform sequence is defined at the well location, which is maintained along the sequence.

- The method has strict requirements to the data, i.e., the availability of at least one well in the target zone of the clinoform sequence. The required logs are sonic and gamma ray logs. A density log is preferable for more accurate results but not mandatory. A poststack (time or depth) migrated 2D seismic section is required.

- The starting point for the pseudo 2D inversion process is from the closest trace to the well.

\subsection{Grid-based Seismic Inversion}

The innovation of this method is the implementation of a technique recently introduced by Van Eekeren et al. (2008) into a seismic inversion setting.

Van Eekeren et al. (2008) introduced a super-resolution (SR) reconstruction of small moving objects from a low-resolution undersampled image sequence, which we used here to characterize the clinoform 
data set. To this end, the original objective function was modified by adding two additional terms, i.e., the vertical and the horizontal operator. The vertical operator favored an inversion solution, with sparse layering. The horizontal operator favored continuity of the inversion results in the horizontal direction.

Similar to the stratigraphic model-based method, this method integrated the well data, that have a high vertical resolution, and the seismic data that have a high lateral resolution. The resulting inversion model is a consistent, 2-D high-resolution impedance model of the clinoform sequence.

The implementation of the hyperbolic norm with a first and a second order derivative that exist everywhere allowed us to use the more sophisticated Levenberg-Marquardt optimization method.

Minimization of the modified version of the cost function, employed in the current method, is still an ill-posed problem. Similar to the previous inversion method, the ill-posed nature of the inversion technique was handled by regularization of the inverse problem. The modified version of the cost function contained several terms that needed to be regularized. The regularization was applied in an empirical manner. The understanding of the physical background of the weighting coefficients and their relationships helped steering the updates. In addition, extensive tests on the sensitivity of the parameters were performed to provide information regarding mutual behavior of the parameters. Similar to the stratigraphic model-based method, this regularization technique made the inversion method being driven by the geology.

This seismic inversion method, by adopting the multi-frame superresolution reconstruction method of small moving objects, integrates the seismic data and the well data. It showed encouraging results 
when applied to a clinoform sequence from the North Sea. Similar to the results of the previous method, the acoustic properties of the extracted internal structures of the sequence were at the sub-seismic scale. The estimated details (layers) had thicknesses up to $1 / 10^{\text {th }}$ of the seismic wavelength. The high resolution 2D geological model of the clinoform reservoir was created based on the estimated acoustic parameters.

\section{Advantages}

- The method employs the Total Variation criterion that enforces sparse inversion solutions and thereby allows to present results as a 2D impedance layered model of the clinoform reservoir.

- The cost function of the method includes the horizontal operator that provides sufficient propagation of well data from trace to trace along the clinoform sequence starting from the trace closest to the well.

- The forward modeling technique used in the method has the capability to increase the level of detail of the resulting 2D geological model to a higher level. The vertical resolution of the seismic data was increased by incorporating the well data.

- The method does not require an interpretation of the well data in order to create the 1D initial model of the subsurface. Instead, a well log (impedance in our case) is sampled with a constant time step. As a consequence, the constant time step sampling of the well log prevents the user-introduced bias to occur and greatly simplifies the inversion process.

- The method overcomes the main limitation of the previous method, namely, it permits the fluctuation in the number of strata that 
can be interpreted from the resulting impedance model of the clinoform sequence. This results in more realistic 2D geological models of the clinoform sequence and makes this method more data-driven.

- The method also overcomes another important limitation of the stratigraphic model-based method, because there is no strong restriction in the application of the method concerning the sedimentary environment of the data set.

\section{Limitations}

- The structural geological information (the estimated clinoform shape) is no longer used explicitly in the method, but only implicitly (provided by the horizontal operator).

- The trace sampling of the well log and the acoustic impedance model assumes many samples inside a single layer, i.e., per trace the actual number of layers is much smaller than the parameterized number of samples.

- Upscaling (smoothing) method of the well data performed in Chapter 3 (Section 3.6.2), could be a potential source of error when finely layered media with detail far below the seismic resolution are studied (simple filtering and re-sampling change the gross behavior of the finely detailed medium). For correct upscaling of detailed log information, effective medium theory should be applied (this topic was beyond the scope of this project). The industry standard method is Backus averaging of moduli. For more information, see Lindsay and van Koughnet (2001).

- The wavelet estimation was done with a zero phase assumption, for simplicity. The wavelet was later checked by visual comparison of the synthetic (the well data (reflectivity) convolved with 
the estimated wavelet) and the real, close to the well, seismic traces. The good correlation between these two traces was explained by the assumption that the real data set could have been filtered with the zero-phase filter during the processing stage. In practice, the assumption of a zero phase wavelet is not always valid and the wavelet (including the wavelet scaling) needs to be thoroughly checked by tying the wells to the seismic. This is especially the case when finely layered media are studied.

- Similar to the stratigraphic model-based method, this method has strong data requirements, i.e., it needs at least one well in the target zone of the clinoform sequence, the compulsory logs are sonic and gamma ray logs, with a density log preferable for more accurate results, and a poststack (time or depth) migrated $2 \mathrm{D}$ seismic section.

- The regularization technique used in the method leads to inversion results that contain a certain degree of subjectivity.

- Similar to the previous method, the starting point of the pseudo $2 \mathrm{D}$ inversion process has to be the trace closest to the well.

- The forward modeling technique in this method needs a parametrization of the clinoform sequence with a large number of parameters, and therefore this method has a high computational cost.

\subsection{Geological Significance}

Both impedance models reveal that the sediments with higher impedances are found at the bottom of the sequence (except for the part that was not modeled explicitly in the stratigraphic model-based method (TWT 866-894 ms)). This can be explained by an increase in the degree of 
shaliness of these layers, in line with the findings from the well model, according to which the shales have lower impedances than the sandrich layers. Moreover, an alternation of sand- and shale-rich sediments is observed in both impedance models, and they correlate well with each other. This testifies to the heterolithic nature of such clinoform sequences, and the results may form a useful tool to identify sand-rich layers that might form potential reservoirs.

Thus, the 2D static impedance model obtained from the stratigraphic model-based low parametrization inversion method can provide valuable quantitative information on changes in reservoir quality across the field, but, in comparison, the model-free seismic inversion method with sparsity promotion provides far more accurate quantitative information about such possible reservoir layers.

Both types of impedance models could potentially improve reservoir management through proper drilling strategies, reduced economic risks, increased recovery, and more reliable reserve calculations.

\subsection{Recommendations for further research}

In view of the foregoing, we have three recommendations and several suggestions for improvements on the methods for further research.

First of all, a so-called blind test is advised, where the results of the inversion are validated at well(s), that were not involved in the inversion procedure. Due to the absence of a second well in the area of interest of this study such a blind test could not be performed to validate the methods.

Second, we suggest to apply an industrial inversion package to perform 
an inversion on this clinoform sequence. This test can expose the merits and shortcomings of the methods presented here and at the same time serve as an additional validation criterion of the inversion results.

Third, we recommend to apply a more rigorous quality control (QC) procedure of the well log synthesis, the forward modeling and the quality of the inversions, which was beyond the scope of this project but undoubtedly plays an important role in the reservoir characterization workflow.

In general, we believe that the integration of the two methods has the best potential to improve the results of the seismic inversion procedure.

In cases where more than one well is available in the area of interest we recommend to account for the a priori uncertainties in a more accurate way. Instead of using the standard deviation of the best-fit Gaussian distribution to the histogram of the single acoustic impedances log to estimate the uncertainties for every layer (stratigraphic model-based method) or every sample (model-free method), several logs should be involved, thereby making it possible to estimate the uncertainties for each layer (or sample) individually.

Apart from these more general remarks, we propose several improvements that are explicitly aimed at the two methods.

\section{Stratigraphic model-based, low parametrization seismic in- version}

- Since the influence of the well data on the inversion results decreases with the distance from the well, we advise to make the regularization parameter $\lambda$ variable along the clinoform sequence, having the highest impact in the beginning. 
- A compaction effect of the sediments was detected in the well data, i.e., the deeper sediments are characterized by higher acoustic impedance values (Chapter 3, Section 3.6.2). A 'detrending procedure' of the log data is advised to compensate the compaction effect.

- The method can be extended to areas where the clinoform shapes are revealed only partially on the seismic image due to the low vertical resolution of the seismic data. By using the predictive capabilities of the method, those partly exposed clinoform shapes still can be estimated and integrated into the inversion procedure. However, in this case we advise to use them as soft constraints that will guide the 1D inversion, rather than as hard boundaries.

- Because no significant changes in the geological settings are expected between neighboring seismic traces (spaced at $25 \mathrm{~m}$ ), we propose to modify the original cost function by adding a third term that regularizes the amount of variation in the properties allowed from trace to trace.

- In the current state of the method the initial model, called 'Well Model', is constructed based on log analysis of the lithofacies and their layering, causing a certain degree of user bias. In order to increase the confidence in the initial model, the number of layers in the clinoform sequence that are identified in the model-free seismic inversion method can serve as an additional constraint in the 'Well Model' construction.

\section{Grid-based Seismic Inversion}

- Structural geological information is not used explicitly in this method, but only implicitly via the horizontal operator. We 
propose to extend or replace this horizontal operator with an operator that incorporates the structural information provided by the estimated clinoform models. This new operator also should favor sparse solutions, but now in the direction of the clinoforms.

- We recommend to make the method a full 2D inversion procedure. For the horizontal operator, or the new geologically driven operator, it makes more sense to use several traces or even the entire clinoform sequence. As a 2D initial model, one can use the static impedance model obtained by optimizing the stratigraphic model-based inversion method.

- Since there is no restriction in applying the method to other geologic settings, expanding the method to other geological macrostructures, e.g., channels can be recommended 


\section{Bibliography}

Alliney, S. and Ruzinsky, S., 1994. An algorithm for the minimization of mixed $l_{1}$ and $l_{2}$ norms with application to bayesian estimation. IEEE Transactions on Signal Processing, 42(3):618 -627.

Aminzadeh, F. and de Groot, P., 2006. Neural networks and other soft computing techniques with applications in the oil industry. EAGE publications BV, Houten, The Netherlands.

Amundsen, L., 1991. Comparison of the least squares criterion and the Cauchy criterion in frequency-wavenumber inversion. Geophysics, 56:2027Ü-2035.

Badley, M. E., 1985. Practical Seismic Interpretation. Prentice Hall, Englewood Cliffs, New Jersey.

Bard, Y., 1973. Nonlinear parameter estimation. Academic Press, New York.

Barnett, V., 1982. Comparative Statistical Inference. John Wiley and Sons, 2nd edition.

Bosch, M., Carvajal, C., Rodrigues, J., Torres, A., Aldana, M., and Sierra, J., 2009. Petrophysical seismic inversion conditioned to welllog data: Methods and application to a gas reservoir. Geophysics, $74(2): 1-15$. 
Cacas, M.-C., Laigle, J.-M., Albouy, E., and Sulzer, C., 2008. Integrating stratigraphic modelling and seismic interpretation: two practical examples. Petroleum Geoscience, 14:253-261.

Cary, P. and Chapman, C. H., 1988. Automatic 1-D waveform inversion of marine seismic refraction data. Geophysical Journal International, 105:289-294.

Cattaneo, A., Trincardi, F., Correggiari, A., and Carra, D., 2003. Clinoform construction in subaqueous deltas: An example from the adriatic sea (italy). Geophysical Research Abstracts, European Geophysical Society, 5:11277.

Cattaneo, A., Trincardi, F., Langone, L., Asioli, A., and Puig, P., 2004. Clinoform generation on Mediterranean margins. Oceanography, 17(4):104-117.

Clausen, O. and Korstgard, J., 1993. Small-scale faulting as an indicator of deformation mechanism in the tertiary sediments of the northern Danish central trough. Journal of Structural Geology, 15(2):1343-1357.

Crase, E., Pica, A., Noble, M., McDonald, J., and Tarantola, A., 1990. Robust elastic nonlinear waveform inversion: Application to real data. Geophysics, 55(5):527-538.

Debeye, H. W. J. and van Riel, P., 1999. $L_{p}$-norm deconvolution. Geophysical Prospecting, 38:381 -403.

Duijndam, A. and Drijkoningen, G., 1997. Parametric inversion with seismic applications. Delft Technical University.

Duijndam, A. J. W., 1988. Bayesian estimation in seismic inversion, part 1: Principles. Geophysical Prospecting, 36:878-898. 
Efron, B., 1986. Why isn't everyone a Bayesian? The American Statistician, 40(1):1-5.

Emery, D. and Myers, K., 2005. Sequence Stratigraphy. Blackwell Science, Oxford.

Farsiu, S., Robinson, M. D., Elad, M., and Milanfar, P., 2004. Fast and robust multiframe super resolution. IEEE Transactions on image processing, 13(10):1327-1344.

Gill, P. E., Murray, W., and Wright, M. H., 1982. Practical Optimization. Academic Press, New York.

Goutis, C. and Casella, G., 1995. Frequentist post-data inference. International Statistical Review, 63(3):325-344.

Gregersen, U., 1997. Sequence stratigraphic analysis of Upper Cenozoic deposits in the North Sea, based on conventional and 3-D seismic data and well-logs. Ph.D. thesis, University of Aarhus, Aarhus.

Griva, I., Nash, S. G., and Sofer, A., 2009. Linear and Nonlinear Optimization. SIAM, Philadelphia (PA), USA, second edition.

Huber, P., 1981. Robust Statistics. Wiley.

Jackson, D. D., 1972. Interpretation of inaccurate, insufficient and inconsistent data. Geophysical Journal of the Royal Astronomical Society, 28:97-109.

Kay, C., 1993. The growth and gross morphology of Quaternary deltas in the southern North Sea. Ph.D. thesis, University of Edinborough.

Kenter, J., 1990. Carbonate platform flanks: slope angle and sediment fabric. Sedimentology, 72(1):777-794. 
Lailly, P., 1983. The seismic inverse problem as a sequence of before stack migrations. Proceedings of the international conference on 'Inverse Scattering, theory and applications', Tulsa, Oklahoma, SIAM Publisher.

Li, Y. and Santosa, F., 1996. A computational algorithm for minimizing total variation in image restoration. IEEE Transactions on image processing, 5(6):987-995.

Lindley, D. V., 1975. The future of statistics: A Bayesian 21st century. Advances in Applied Probability. Supplement: Proceedings of the Conference on Directions for Mathematical Statistics, 7:106-115.

Lindsay, R. and van Koughnet, R., 2001. Sequential backus averaging: Upscaling well logs to seismic wavelengths. The Leading Edge, 20:188-191.

Luthi, S. M., 2001. Geological Well Logs, their use in reservoir modeling. Springer-Verlag Berlin Heidelberg, Germany, 1st edition.

Luthi, S. M., 2008. Fossil hydrocarbons: Where, how much and for how long? In D.-J. Peet, C. Hatton, G. de Werk, and R. van Drimmelen, editors, Geotechnology and sustainable develpment, pages 1424. Eburon Delft.

Marzuki, Z. B., Sams, M. S., and Atkins, D., 2000. Improving the static model of a complex reservoir through the inversion of seismic data. Technical Report 64740, Society of Petroleum Engineers.

Merletti, G. D. and Torres-Verdin, C., 2006. Accurate detection and spatial delineation of thin-sand sedimentary sequences via joint stochastic inversion of well logs and 3D pre-stack seismic amplitude data. Technical Report 102444, Society of Petroleum Engineers. 
Milton, N. J. and Bertram, G. T., 1995. Topset play types and their controls. In J. C. van Wagoner and G. T. Bertram, editors, Sequence Stratigraphy of Foreland Basin Deposits, Outcrop and Subsurface Examples from the Cretaceous of North America, volume 64, pages 1-9. American Association of Petroleum Geologists.

Moré, J. J., 1978. The Levenberg-Marquardt Algorithm: Implementation and Theory, volume 630 of Lecture Notes in Mathematics, pages 105-116. Springer Berlin / Heidelberg.

Nelder, J. A. and Mead, R., 1965. A simplex method for function minimization. The Computer Journal, 7(4):308-313.

Pendrel, J., 2001. Seismic inversion - the best tool for reservoir characterization. Technical report, Fugro-Jason.

Pendrel, J. V. and van Riel, P., 1997. Methodology for seismic inversion and modeling: A western Canadian reef example. CSEG Recorder, 25(5):5-15.

Press, W. H., Teukolsky, S. A., Vetterling, W. T., and Flannery, B. P., 2007. Numerical recepies. The art of scientific computing. Cambridge University Press, New York, USA, 3rd edition.

Rich, J. L., 1951. Three critical environments of deposition, and criteria for recognition of rocks deposited in each of them. Geological Society of America Bulletin, 62(1):1-20.

Rubin, D. B., 1984. Bayesianly justifiable and relevant frequency calculations for the applies statistician. Annals of statictics, 12(4):1151-1172.

Rudin, L. I., Osher, S., and Fatemi, E., 1992. Nonlinear total variation based noise removal algorithms. Physica D, 60:259-268. 
Scales, J. A. and Tenorio, L., 2001. Prior information and uncertainty in inverse problems. Geophysics, 66(2):389-397.

Scales, L., 1985. Introduction to nonlinear optimization. Macmillan.

Schroot, B. M., Klaver, G. T., and Schuttenhelm, R. T. E., 2005. Surface and subsurface expressions of gas seepage to the seabed examples from the southern north sea. Marine and Petroleum Geology, 22(4):499-515.

Shannon, C. E., 1948. A mathematical theory of communication. Bell System Technical Journal, 27:379-423 and 623-656.

Sheriff, R. E., 2002. Encyclopedic Dictionary of Applied Geophysics. Society of Exploration Geophysics, Tulsa, Oklahoma, USA, 13 edition.

Snieder, R., 1998. The role of nonlinearity in inverse problems. Inverse Problems, 14:387-404.

Sørensen, J. C., Gregersen, U., Breiner, M., and Michelsen, O., 1997. High-frequency sequence stratigraphy of upper cenozoic deposits in the central and southeastern north sea areas. Marine and Petroleum Geology, 14(2):99-123.

Steeghs, P., Overeem, I., and Tigrek, S., 2000. Seismic volume attribute analysis of the cenozoic succession in the 108 block (southern North sea). Global and Planetary Change, 27:245-262.

Tan, S. and Fox, C., 1996. Physics 707 inverse problems. University of Auckland (lecture notes), http://www.phy.auckland.ac.nz/Staö/smt/453707SC.html.

Tarantola, A., 1984. Inversion of seismic reflection data in the acoustic approximation. Geophysics, 49(8):1259-1266. 
Tarantola, A., 2004. Inverse Problem Theory and Methods for Model Parameter Estimation. SIAM, Philadelphia (PA), USA.

Tarantola, A., 2005. Inverse problem theory and Methods for model parameter estimation. Society for Industrial and Applied mathematics (SIAM), Philadelphia.

Tarantola, A. and Valette, B., 1982a. Generalized nonlinear inverse problems solved using the least squares criterion. Reviews of Geophysics and Space Physics, 20:219-232.

Tarantola, A. and Valette, B., 1982b. Inverse problems = quest for information. Journal of Geophysics, 50:159-170.

Treitel, S. and Lines, L., 2001. Past, present, and future of geophysical inversion-a new millennium analysis. Geophysics, 66(1):21-24.

Ulrych, T. J., Sacchi, M., and Woodbury, A., 2001. A Bayes tour of inversion: A tutorial. Geophysics, 66(1):55-69.

van Boogaert, H. A. A. and Kouwe, W. F. P., 1993. Nomenclature of the Tertiary of the Netherlands. RGD \& NOGEPA. 50, The Netherlands, 2nd edition.

van Eekeren, A. W. M., Schutte, K., and van Vliet, L. J., 2008. Multiframe super-resolution reconstruction of small moving objects. submitted.

van Riel, P., 2000. The past, present, and future of quantitative reservoir characterization. The Leading Edge, 19(8):878-881.

van Riel, P. and Mesdag, P. R., 1988. Detailed interpretation of the North sea Magnus field by integration of seismic and well information. SEG Expanded Abstracts, 7(1):869 -872. 
van Riel, P. and Pendrel, J., 2000. Effect of well control on constrained sparse spike seismic inversion. Recorder, 25(12):18 -26.

Vetterli, M., Marziliano, P., and Blu, T., 2002. Sampling signals with finite rate of innovation. IEEE Transactions on Signal Processing, 50(6):1417-1428.

Widess, M. B., 1973. How thin is a thin bed? Geophysics, 38(6):11761254 .

Woods, D. J., 1985. An interactive approach for solving multi-objective optimization problems. Ph.D. thesis, Rice University, Houston, Texas.

Yilmaz, O., 2000. Seismic data analysis: processing, inversion and interpretation of seismic data. Society of Exploration Geophysics, Tulsa, USA, 2nd edition.

Ziegler, P., 1988. Evolution of the arctic-north atlantic and western tethys. American Association Petroleum Geologists, 43.

Ziegler, P., 1990. Geological atlas of Western and Central Europe. Shell Internationale Petroleum Maatschappij B.V, Geological Society of London, Elsevier, Amsterdam, 2nd edition. 


\section{About the author}

Daria Tetyukhina was born in Moscow, Russia, on the 24th of June 1982. She graduated from the Gymnasium of Physics and Mathematics in Moscow in 1999. In the same year she started her study Geophysics at the faculty of geology at Lomonosov Moscow State University. She obtained her BSc in Geology, specialisation in Geophysics, with honors in 2003. The BSc thesis was entitled 'Method of processing marine high-frequency multi-channel seismic data'. The results of this research were presented during the 'SEG EAGE EAGO International Geophysical Conference and Exhibition 2003', where, she participated in the organizing committee as a students' program manager.

The same year she started her MSc study. The results of the MSc projects served as a basis for presentations at several Russian conferences as well as for EAGE (2004) and SEG (2005). During her study, Daria was actively participating in the board of the SEG EAGE student chapter of the Lomonosov Moscow State University from 20032005. In 2002 and 2004 she did a summer school at Hampstead School, London. During her MSc, from 2003-2005, she worked as a part-time geophysicist at Deco Geophysical. She obtained her MSc in Geology, specialisation in Geophysics with honors in July 2005. The MSc thesis was entitled 'Use of multi-channel, high-resolution, shallow water seismic data for subsurface sediments characterization'. 
In August 2005, Daria joined Delft University of Technology as a PhD student. Her $\mathrm{PhD}$ project entitled 'High-resolution reservoir characterization by seismic inversion with geological constraints' is an interdisciplinary project between the Applied Geophysics and the Applied Geology groups of the Department of Geotechnology, and the Quantitative Imaging group of the Department of Imaging Science and Technology. During her PhD program Daria contributed to some international conferences such as EAGE (2006, 2007, 2010) SEG (2007, 2008) and AAPG 2009. She visited Statoil several times to present her research results and to attend an internal course on geological modeling.

Since 2006, Daria is part of the SEG Global Affair Committee and as from 2008 she is a Task Force Leader (Student Chapters, Activities and Competitions) within the committee. She was on the founding board of the SEG student chapter at Delft University of Technology and served as vice-president in the period of 2007-2009. She is also a member of EAGE, AAPG and SPE.

As from January 2010, Daria works as a Postdoctoral researcher at Delft University of Technology in a joint project between the Delphi Consortium and the Department of Geotechnology. 


\section{List of Publications}

\section{Publications}

Tetyukhina, D., L. J. van Vliet, S. M. Luthi, and K. Wapenaar, 2010, High-resolution reservoir characterization by an acoustic impedance inversion of a Tertiary deltaic clinoform system in the North Sea: Geophysics. (accepted).

Tetyukhina, D., L. van Vliet, K. Wapenaar, and S. M. Luthi, 2010, 2D high-resolution reservoir characterization by model-free seismic inversion with sparsity promotion: 72nd EAGE Conference \& Exhibition incorporating SPE EUROPEC, Barcelona, Spain, 14-17 June, Extended Abstracts, F028.

Tetyukhina, D., S. M. Luthi, L. J. van Vliet, and K. Wapenaar, 2008, High-resolution reservoir characterization by 2-D modeldriven seismic Bayesian inversion: an example from a tertiary deltaic clinoform system in the North Sea: SEG 78th Annual Meeting, Las Vegas (NV), USA, 9-14 November, Expanded Abstracts, 27, 1880-1884.

Tetyukhina, D. D., L. J. van Vliet, S. M. Luthi, and C. P. A. Wapenaar, 2007, High resolution thin-bed inversion based on the Bayesian approach: 69th EAGE Conference \& Exhibition incor- 
porating SPE EUROPEC, London, UK, 11-14 June, Extended Abstracts, P357.

Tetyukhina, D., L. J. van Vliet, S. M. Luthi, and C. P. A. Wapenaar, 2007, High-resolution clinoform characterization by 2-D model-driven seismic Bayesian inversion: SEG 77th Annual Meeting, San Antonio (TX), USA, 23-28 September, Expanded Abstracts, 26, 1855-1859.

Tetyukhina, D., 2005, Use of multi-channel high resolution shallow water seismic data for subbottom sediment characterization: SEG 75th Annual Meeting, Houston (TX), USA, 6-11 November, Expanded Abstracts, 1164-1167.

\section{Conference Proceedings}

Tetyukhina, D., S. M. Luthi, L. J. van Vliet, and K. Wapenaar, 2009, 2-D high-resolution reservoir characterization by seismic bayesian inversion of a tertiary deltaic clinoform system in the North Sea: Presented at AAPG Annual Convention \& Exhibition, Denver (CO), USA, 7-10 June.

Tetyukhina, D. D., S. M. Luthi, L. J. van Vliet, and K. Wapenaar, 2008, High-resolution characterization of the North Sea fluvio-deltaic system by seismic Bayesian inversion: Presented at the 9th Nederlands Aardwetenschappelijk Congres, Veldhoven, The Netherlands, 18-19 March.

Tetyukhina, D. D., 2006, The department of Geotechnology at TU Delft. An example: High-resolution reservoir characterization project (PhD project): Presented at EAGE Saint Petersburg 2006, Saint Petersburg, Russia, 16-19 October, Students' Forum. 
Tetyukhina, D., 2005, The possibility of AVO analysis of a seismic data at the permafrost areas in the West Siberia: Presented at Geomodel 2005, the 7th annual conference and workshop, Gelendzhik, Russia, 11-17 September (in Russian).

Tetyukhina, D., 2004b, Processing method of marine high frequency multichannel seismo-accoustical data: Presented at the 66th EAGE Conference \& Exhibition incorporating SPE EUROPEC, Paris, France, 7-10 June, Students' Forum.

Tetyukhina, D., 2004a, Corrections of the acquisition positioning of marine high-frequency multi-channel seismic data: Presented at The Young Scientists' Section of Geomodel 2004 International Conference and Workshop, Moscow, Russia, 15-16 April (in Russian).

Kalmikov, D. K., R. A. Nikonov, S. A. Polyanov, and D. D. Tetyukhina, 2004, Comparison of the results of the single-channel and multichannel seismoacoustic observations based on materials of the marine field trip 2004: Presented at Geomodel 2004, the 6th annual conference and workshop, Gelendzhik, Russia, 12-18 September (in Russian).

Tetyukhina, D., 2003, Processing method of marine high-frequency multichannel seismoacoustical data: SEG EAGE EAGO International Geophysical Conference \& Exhibition, Moscow, Russia, 1-4 September (in Russian). 


\section{Acknowledgments}

I would like to take this opportunity to thank all the people who in some way have helped me during my $\mathrm{PhD}$, and without whom starting and completing this thesis would have been impossible.

I am very grateful to my promotors, Stefan Luthi, Lucas van Vliet, and Kees Wapenaar, for their guidance and help throughout my PhD study. I am very happy that I got a unique opportunity to work with supervisors with three different backgrounds, giving me a chance to observe different ways of thinking and approaching problems, but at the same time being able to make a process of solving a problem clearly and understandably for everyone. I have learned a lot from them. I am very thankful for all the time they have spent helping me to achieve my goals, and for their thorough reviews of everything I wanted to publish or present. This thesis greatly benefited from your contribution and I highly acknowledge your very thorough editorial work while preparing this thesis.

I would like to acknowledge the Research Center Delft Earth and Statoil for sponsoring this research. Particular thanks go to Steen Petersen for his help and useful discussions. 
I am very happy that I got an opportunity to be a part of three different groups at the same time and work with so many people. Not to forget anyone, since the list is quite long, I would like to collectively thank all my colleagues, former and present, for all their help and support. Special thanks to them for the conference trips we made during this time, which always were great fun, and for all parties we organized.

I would also like to thank my former colleagues from the Lomonosov Moscow State University for introducing me to the geoscience world.

I am very much indebted to Bobby for providing me with enormous help during my PhD project, I could not do without, as well as in the preparation of this thesis. I also want to thank you for your tremendous support.

I wish to thank my family for all their love and support. Especially, I would like to thank my grandfather Vladislav who brought the idea of $\mathrm{PhD}$ into my head while chatting on the ski lift in the mountains. Thanks to my aunt Vera and her family for their love, support and always warm welcome. I want to thank my little sister Kate, who is not little anymore, but for me always will be, for all the joy she brings into our life!

There are no words to express my gratitude to my parents Nadezhda and Dmitry, who have always believed in me and stood behind me whatever happened... Everything I have ever achieved in my life, including this $\mathrm{PhD}$, is because of their infinite love and support. Thanks... 
Я хотела бы поблагодарить людей, вклад которых в мою жизнь неизмерим - свою Семью. Спасибо за Вашу поддержку и любовь, которые Вы мне дарите!

Дед, спасибо тебе огромное, я думаю, ты даже не подозревал, к чему это приведет, когда случайно предложил мне идею аспирантуры на подъемнике, когда мы катались на лыжах! Моя любимая Вера, спасибо тебе и твоей семье за всю Вашу любовь и поддержку!

Моя любимая сестра, спасибо тебе за всю ту радость, которую ты привносишь в нашу жизнь!

Я думаю, что я вряд ли смогу найти слова, которыми я смогла бы поблагодарить Вас, моих любимых родителей, за все то, что Вы сделали и продолюаете делать для меня, за Вашу любовь и поддержку. Все, чего я достигла в своей жсизни, включая эту диссертачию - это благодаря Вам, Вашей безграничной любви, безмерной поддерэке и нескончаемой вере в меня.

Спасибо... 


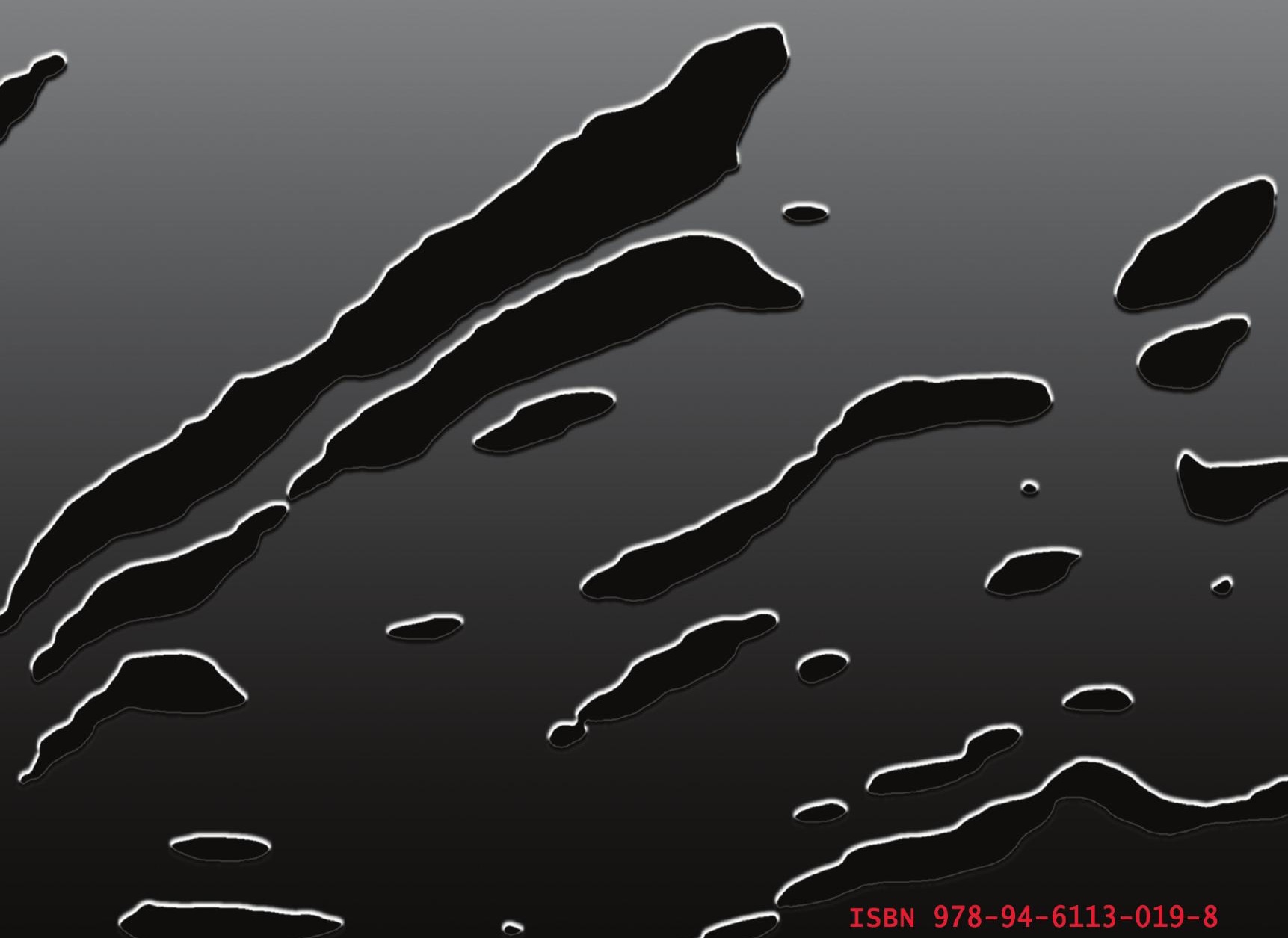

